\title{
Dynamic modeling and simulation of an integral bipropellant propulsion double-valve combined test system
}

\author{
Yang Chen ${ }^{\mathrm{a}, *}$, Huasheng Wang ${ }^{\mathrm{b}}$, Jixia Xia ${ }^{\mathrm{c}}$, Guobiao Cai ${ }^{\mathrm{a}}$, Zhenpeng Zhang ${ }^{\mathrm{a}}$ \\ ${ }^{\text {a }}$ School of Astronautics, Beihang University, Beijing, 100191, China \\ ${ }^{\mathrm{b}}$ School of Engineering and Materials Science, Queen Mary, University of London, London, E1 4NS, UK \\ ${ }^{\mathrm{c}}$ Beijing Institute of Control Engineering, Beijing, 100190, China
}

\begin{abstract}
For the pressure reducing regulator and check valve double-valve combined test system in an integral bipropellant propulsion system, a system model is established with modular models of various typical components. The simulation research is conducted on the whole working process of an experiment of 9MPa working condition from startup to rated working condition and finally to shutdown. Comparison of simulation results with test data shows: five working conditions including standby, startup, rated pressurization, shutdown and halt and nine stages of the combined test system are comprehensively disclosed; valve-spool opening and closing details of the regulator and two check valves are accurately revealed; the simulation also clarifies two phenomena which test data are unable to clarify, one is the critical opening state in which the check valve spools slightly open and close alternately in their own fully closed positions, the other is the obvious effects of flow-field temperature drop and temperature rise in pipeline network with helium gas flowing. Moreover, simulation results with consideration of component wall heat transfer are closer to the test data than those under the adiabatic-wall condition, and more able to reveal the dynamic characteristics of the system in various working stages.
\end{abstract}

Keywords: Integral bipropellant propulsion; Double-valve combined test system; Finite volume method; Modularization modeling; Dynamic system simulation

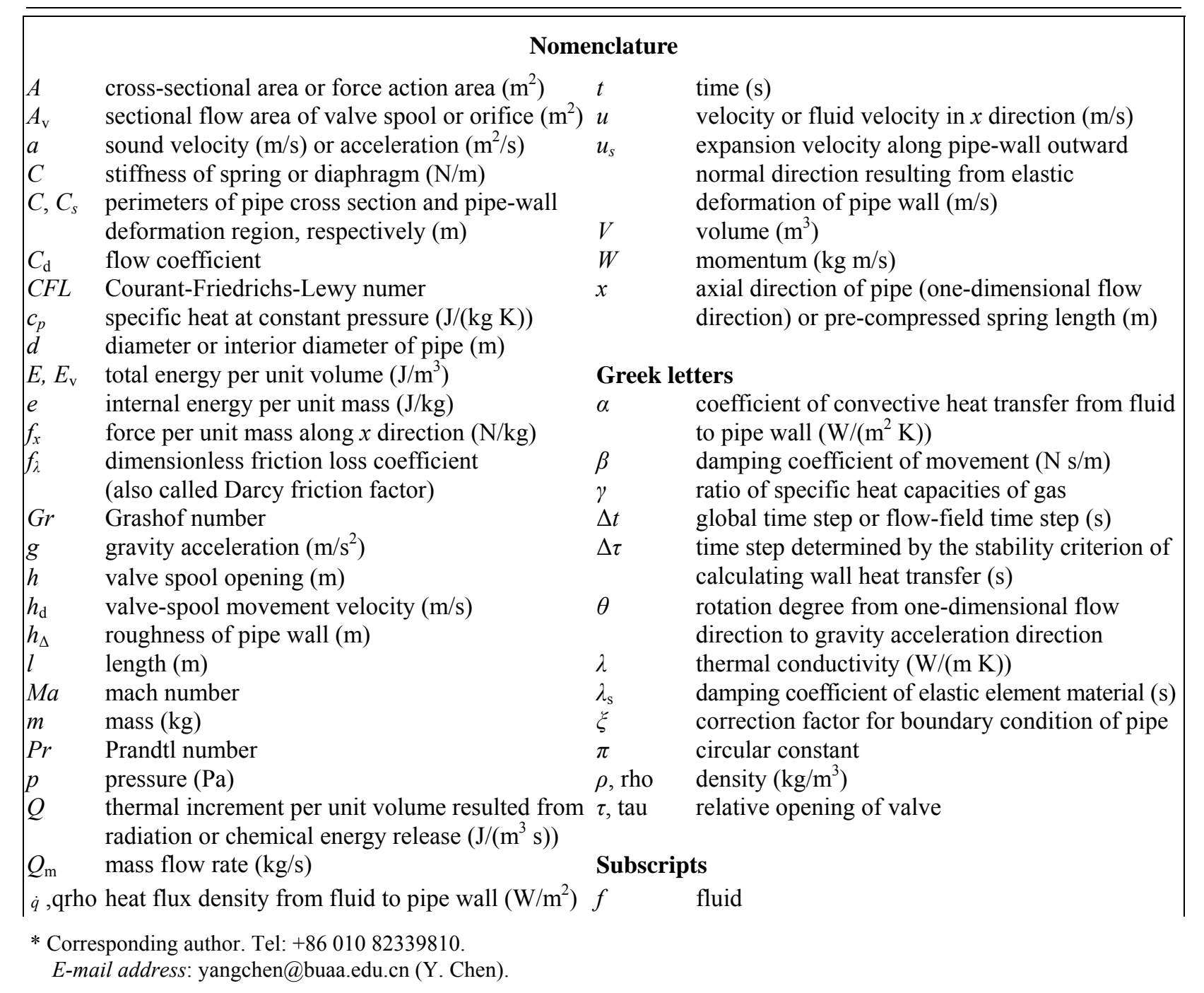




\begin{tabular}{|c|c|c|c|}
\hline qrho2 & $\begin{array}{l}\text { heat flux density from exterior wall to } \\
\text { environment }\left(\mathrm{W} / \mathrm{m}^{2}\right) \\
\text { specific gas constant }(\mathrm{J} /(\mathrm{kg} \mathrm{K}))\end{array}$ & $i, j, k$ & $\begin{array}{l}\text { serial numbers of flow-field state element, } \\
\text { velocity element, and wall radial-direction grid, } \\
\text { respectively }\end{array}$ \\
\hline$R a$ & Rayleigh number & in, out & inlet and outlet of grid, respectively \\
\hline $\operatorname{Re}$ & Reynolds number & $n$ & total number of flow-field grids \\
\hline & pipe-wall radial coordinate or radius (m) & $n_{w}$ & total number of wall temperature-field \\
\hline$r_{s}$ & radial coordinate of wall corresponding to the & & radial-direction grids \\
\hline & barycentre of control volume (m) & $\mathrm{w}, \mathrm{e}, \mathrm{s}, \mathrm{n}$ & western, eastern, southern and northern \\
\hline$S, S_{s}$ & $\begin{array}{l}\text { interior surface areas of wall in control-volume } \\
\text { region and deformation region, respectively }\left(\mathrm{m}^{2}\right)\end{array}$ & & $\begin{array}{l}\text { boundaries of the temperature field grid element, } \\
\text { respectively }\end{array}$ \\
\hline$T, T_{w}$ & temperature and interior-wall temperature $(\mathrm{K})$ & $w$ & wall \\
\hline
\end{tabular}

\section{Introduction}

Pressure reducing regulator (PRR) and check valve (CV) are two types of components widely applied to various liquid propulsion systems and energy systems [1-9]. The former is used to reduce the pressure of inlet high-pressure fluid to the defined value and stabilize it within a certain range; the latter is used to prevent reverse flow. The double-valve combined test system in this paper is the ground test system of an integral bipropellant propulsion tank pressurization system. Its function is to check whether the performance of the two types of key components in the pressurization system can meet the design requirement before the propulsion system whole-system test. Common bipropellant propulsion tank pressurization systems [5-9] generally contain the PRR in the main pipeline of gas system and the CV in the tank pressurization pipeline. As other valves usually have no action any more since system startup up to system shutdown, the two types of key components are often a limited number of movable components in the gas system during the operation of propulsion system. Earlier numerical researches [7-10] use relatively simple models. In the most simplest models [8], the PRR inlet pressure is given by the gas-bottle model and the outlet pressure is designated rather than obtained by real-time calculation based on flow conservation equations and force equilibrium equation; the $\mathrm{CV}$ model only calculates the pressure friction loss and considers its value as the function of helium flow rate. In the modeling for Galaxy XII dual mode bipropellant propulsion subsystem [9], the PRR model uses function expression of outlet pressure changing with inlet pressure and temperature; the CV model also only considers the pressure loss. The expression is determined by test data rather than the characteristics model established based on the PRR structure, so the model cannot work out the PRR opening characteristic. Later researches gradually take into consideration dynamic force equilibrium of various chamber fluids and elastic components acting on valve-spool moving part. A summary of classification is given below.

As a component for reducing and stabilizing pressure in the fluid pipeline system, the PRR is widely researched [11-23] for its dynamic characteristics. However, many models for gas PRR adopt some assumptions such as isothermic [12,13], isentropic [14-16] and linearization [15] which do not strictly agree with reality. Their pressure differential equations are usually obtained by taking the derivative of ideal gas state equation or isentropic equation and employing isothermal process assumption [12] or isentropic process assumption [15,16] rather than by deduction from the energy equation of compressible transient flow in one-dimensional (1D) conservative form. The final forms of these models depend too much on ideal gas state equation or isentropic equation; many models either do not give comprehensive consideration to roles of various chambers [12,13,15,16,21] (usually emphasize on simulating throttling and pressure stabilizing of valve spool but give little or inadequate consideration to roles of other chambers than high- and low-pressure chambers) or adopt pressure difference-based relationship between pressure and flow rate for valve spool and orifice throttling [15-20]. Moreover, there are some researches [21-23] on liquid PRR in the hydraulic system which use the method of bond graph and regard the fluids as incompressible flow. In addition, all the abovementioned researches do not consider heat transfer effect of environment and pipe wall on flow fields of various chambers of the PRR, and most of these researches do not conduct modeling for the test system to which the PRR is really applied.

The CV, used for constraining the fluid flow in only one direction and preventing reverse flow, is widely applied to various fluid systems including aerospace propulsion systems [1,3-9], electronic equipment [2], nuclear reactor systems [24-26], compressor station systems [27], mechanical system [28], oil or gas pipeline systems [29], heating, ventilation and air-conditioning (HVAC) systems [30-34], microfluidic systems [35,36] and so on. McElhaney [24] and $\mathrm{Xu}$ et al. [25] classify the check valves applied in the nuclear industry into swing type, lift type, tilting disc type, double discs type, stop type, nozzle type and so on. According to this classification, the CV researched by this paper is a double-spool lift CV in aerospace propulsion system and a dual-poppet series-redundant assembly [6] in structure. McElhaney [24] also gives analysis on structures, advantages and disadvantages of four common check valves including swing $\mathrm{CV}$, lift $\mathrm{CV}$, tilting disc $\mathrm{CV}$, double discs $\mathrm{CV}$ and summarizes such information as failure mode, failure cause, failure discovery method and failure area of all kinds of check valves. One problem with the 
lift $\mathrm{CV}$, when this design is used in air or steam systems, is that severe seat tapping can occur at low flow rates when the disc is only partially open. This cyclic impacting against the seat can cause severe vibration and rapid wear. These four typical check valves have a common feature [25]: the direction of head/disc motion is uniform with flow direction, so the head/disc will be driven by flow when the CV opens or closes.

The abovementioned researches on the CV can be roughly divided into component level and system level. The component-level research has developed into 3D steady-state or transient modeling from 1D steady-state modeling. $\mathrm{Xu}$ et al. [25] point out that numerical simulations of the flow in different check valves have been widely carried out in recent years, and many of these analyses have focused on the ability of different turbulence models to correctly reproduce the features of the complex flows. They conduct 3D simulation and analysis on steady-state hydraulic characteristics of a new type of contra-push CV with the CFD (Computational Fluid Dynamics) software Fluent 6.3 and reveal the valve-spool motion law by transient simulation under different working conditions of pressure. Yu et al [28] employ Fluent 6.3 to conduct 2D axisymmetric simulation and optimization of an axial-flow CV so as to decrease flow resistance and noise. Botros [27] uses Fluent 6.3 to conduct 2D simulation for steady-state flow field of a nozzle CV applied to the compressor station and establishes a two-degree of freedom forced vibration model for the internal valve assembly, which illustrate the associated dynamic phenomena and fluid-structure interaction within the internal assembly of the CV. According to the analysis, the internal geometry of a nozzle CV should be optimized such that the highest possible hydrodynamic drag coefficient is realized when the disk is at its fully open position; if the nozzle CV is used in compressor stations, spring force and stiffness should be selected on the basis of the relationship between the valve flow characteristics at the fully open position in relation to the compressor performance map in order to avoid a partially open valve. In addition, Botros also points out that spring force and stiffness determine the performance of this type of $\mathrm{CV}$ and impact the overall operation and integrity of the compressor station; when the spring forces are higher than the maximum hydrodynamic force at minimum flow, the disk will not be at the fully open position, which will give rise to disk fluttering and potential for cyclic high velocity impact between components of the internal valve assembly. This could lead to self destruction of the CV and subsequent risk of damage to the compressor unit itself.

The component-level research can use high-dimensional CFD method. However, the CFD approach does not enable representation of the whole system, in particular as far as the motions of actuators are concerned [17]. Therefore, the system-level research must reach reasonable balance between accuracy of component models and complexity of the overall system. The general approach is to use the 1D or even 0D simplified model for modeling and simulation, with focus on overall characteristics of the system and the specific role of a single component during the system dynamic change [37]. For the inadequate accuracy problem of earlier swing CV model, Lim et al. [26] improve the system-level CV model with new angular momentum equation describing the swing disc and implement it into the thermal-hydraulic system code, MARS. They establish a node-type model containing 66 hydrodynamic volumes and 68 junctions for a gravity-feed pipeline with 9 swing check valves in a South Korea nuclear power plant and conduct simulation. The effectiveness of the CV model is verified through a comparison of the simulation results with the experimental data. For the problem of excess flow demand and low chilled water temperature difference from which large primary-secondary chilled water systems often suffer during operation, which is known as the low $\Delta \mathrm{T}$ central plant syndrome, Wang and Ma et al. [30] present an approach for experimental validation of possible utility of a bypass CV to solve this operational problem and enhance the overall system performance. They [31] point out that: although the use of the bypass CV has been much concerned in many studies, the well-documented and detailed simulation work or case study demonstrating whether the bypass CV is a good practice that can be used to handle the low $\Delta \mathrm{T}$ syndrome in primary-secondary systems is still lacking. For the problem, they conduct simulation research and the results show that $24997.2 \mathrm{kWh}(6.77 \%)$ total energy of the chilled water system can be saved when the operation of the chilled water system suffered from the $20 \%$ air-side fouling and a bypass CV was used, as compared to that without using the bypass CV in the same condition. The TRNSYS simulation software [32-34] on which the research of Ma and Wang [31] is based has been widely applied to such fields as HVAC systems after more than three decades of development. Its modeling idea is the same as the abovementioned idea, that is, using 1D or even 0D simplified model for system-level modeling and simulation.

Researches [35,36] in microfluidic applications emphasize on design and thus just are relational-expression-level steady-state simulation. In addition, there are also problems in the process of the CV system-level modeling similar to those in the PRR modeling.

In view of the problems of researches on PRR and CV, this paper, based on the proposed unified basic theory [37,38], establish a new type of finite volume model for various chambers and valve-spool moving parts of a high-pressure-unloading diaphragm-type gas PRR and a double-spool lift gas CV, and corresponding finite volume models for other components of the double-valve combined test system. The two kinds of regulators will eventually be applied to the tank pressurization system of a bipropellant propulsion system. The system, as the gas pressurization system adopting the integral mode (i.e. shared by orbit-control and attitude-control engines), is designed to enable the propellant feed system to deliver propellants of corresponding flow rate by pressurizing the 
propellant tanks so as to supply oxidant and fuel for the thrusters. Therefore, only if its dynamic characteristics meet certain requirements in the working process, normal operation of engines under two working modes (orbit-control continuous mode and attitude-control pulse mode) can be guaranteed. As various gas valves in this system do not have action any more after the first startup of system and only play the role of circulation or blocking under two working modes, the dynamic characteristics of this system mainly depend on the PRR and two check valves. The PRR is responsible for reducing the pressure of helium outflowing from high-pressure gas bottle to the design pressure point and stabilizing it within a certain range; two check valves are responsible for preventing the backflow of helium in two propellant-tank pressurization pipelines and controlling the helium flow rates by the throttling of their non-return orifices. Thus the sizes and geometric shapes of flow sections at valve spool of the PRR and big and small valve spools, non-return orifices of two check valves determine the opening, closing and flow characteristics of the system. However, in actual design and test, the stability and dynamic characteristics of the gas system become prominent problems due to complexity of the pipe-network structure, especially to structure complexity of the PRR and CV components in addition to the gas system working under the condition of low flow rate (rated flow: $0.5 \mathrm{~g} / \mathrm{s}$ ). Although the experimental approach can solve the problems, the improvement through experiment takes high costs, long test period and certain operation risks while the computer numerical simulation can provide another research direction for solving the problems at low cost and no operation risk.

Based on the research idea and the PRR stability research results [39], this paper conducts simulation research on the double-valve combined test system to reveal the working characteristics of various components, especially the action details of the PRR and CV at the stages of startup, rated condition and shutdown. Meanwhile, analysis and evaluation on the simulation results are conducted by comparison with experimental data in hope of finally providing a virtual test platform with certain reliability for actual design and experiment.

\section{Modeling approach and verifying tests}

\subsection{Conservation equations and finite volume model of quasi one-dimensional compressible transient pipe flow}

Based on the integral conservation equations in Eulerian type of specification suitable for control volume of continuous fluid medium, the integral and differential conservative equations of quasi one-dimensional compressible transient flow in variable-cross-section pipe [38] can be obtained on condition that $Q=0$ as follows:

$$
\begin{aligned}
& \int \frac{\partial}{\partial t} \int_{x_{1}}^{x_{2}} \rho A \mathrm{~d} x=(\rho u A)_{x=x_{1}}-(\rho u A)_{x=x_{2}} \\
& \left\{\frac{\partial}{\partial t} \int_{x_{1}}^{x_{2}} \rho u A \mathrm{~d} x=\left(\rho u^{2} A+p A\right)_{x=x_{1}}-\left(\rho u^{2} A+p A\right)_{x=x_{2}}+\int_{x_{1}}^{x_{2}} p \frac{\partial A}{\partial x} \mathrm{~d} x+\int_{x_{1}}^{x_{2}} \rho f_{x} A \mathrm{~d} x-\int_{x_{1}}^{x_{2}} f_{R} \rho A \mathrm{~d} x\right. \\
& \frac{\partial}{\partial t} \int_{x_{1}}^{x_{2}} E A \mathrm{~d} x=\left(E u A+p u A-\lambda A \frac{\partial T}{\partial x}\right)_{x=x_{1}}-\left(E u A+p u A-\lambda A \frac{\partial T}{\partial x}\right)_{x=x_{2}}-\left(p u_{s} S_{s}\right)_{r=r_{s}}+\int_{x_{1}}^{x_{2}} \rho f_{x} u A \mathrm{~d} x-\dot{q} S \\
& \frac{\partial \overrightarrow{\boldsymbol{U}}}{\partial t}+\frac{\partial \overrightarrow{\boldsymbol{F}}}{\partial x}=\overrightarrow{\boldsymbol{H}}, \overrightarrow{\boldsymbol{U}}=\left[\begin{array}{l}
\rho A \\
\rho u A \\
E A
\end{array}\right], \overrightarrow{\boldsymbol{F}}=\left[\begin{array}{l}
\rho u A \\
\left(\rho u^{2}+p\right) A \\
(E u+p u-\lambda \partial T / \partial x) A
\end{array}\right], \overrightarrow{\boldsymbol{H}}=\left[\begin{array}{l}
0 \\
p \partial A / \partial x+\rho A\left(f_{x}-f_{R}\right) \\
-p u_{s} C_{s}+\rho f_{x} u A-\dot{q} C
\end{array}\right]
\end{aligned}
$$

In the equations, $f_{R}=f_{\lambda} u|u| /(2 d), f_{x}=g \cos \theta, E=E_{\mathrm{v}}=\rho\left(e+u^{2} / 2\right), \quad S=\int_{x_{1}}^{x_{2}} C \mathrm{~d} x, \quad S_{s}=\int_{x_{1}}^{x_{2}} C_{s} \mathrm{~d} x$, $\dot{q}=\alpha\left(T-T_{w}\right)$. Finally give the state equations of fluid as follows: $p=p(\rho, T), e=e(p, \rho)$

Above equations can be used to analyze quasi one-dimensional compressible transient flow in pipe with variable section on condition of considering the effects including pipe-wall elastic deformation, variable fluid properties, gravitational field, friction, axial heat conduction, heat transfer between fluid and pipe wall. When the volume of the control volume is variable (for example, the fluid cavity of which a part of wall is a mobile piston), the physical meaning of the elastic deformation term in energy equation is the expansion work exported by fluid.

By semi-discretizing equations (1) in two kinds of finite volume grids which are staggered in discrete space as shown in Fig. 1, a finite volume model in form of ordinary differential equations can be established as follows:

1) For state element: $i=0,1, \cdots, n-1$

The continuity equation: $\frac{\mathrm{d}\left(\rho_{i} V_{i}\right)}{\mathrm{d} t}=\rho_{i}^{\text {in }} u_{i} A_{i}-\rho_{i}^{\text {out }} u_{i+1} A_{i+1}$

It can be usually considered that $\left(u_{s} S_{s}\right)_{i}=\left(S_{s} \mathrm{~d} r_{s} / \mathrm{d} t\right)_{i}=\mathrm{d} V_{i} / \mathrm{d} t$, then the energy equation can be: 


$$
\begin{aligned}
& \frac{\mathrm{d}\left(E_{i} V_{i}\right)}{\mathrm{d} t}=\left[\left(E_{i}^{\text {in }}+p_{i}^{\text {in }}\right) u_{i}-\lambda_{i}^{\text {in }} \frac{T_{i}-T_{i-1}}{\bar{x}_{i}-\bar{x}_{i-1}}\right] A_{i}- \\
& {\left[\left(E_{i}^{\text {out }}+p_{i}^{\text {out }}\right) u_{i+1}-\lambda_{i}^{\text {out }} \frac{T_{i+1}-T_{i}}{\bar{x}_{i+1}-\bar{x}_{i}}\right] A_{i+1}-p_{i} \frac{\mathrm{d} V_{i}}{\mathrm{~d} t}+\rho_{i} f_{x, i} V_{i} \frac{u_{i}+u_{i+1}}{2}-\dot{q}_{i} S_{i}}
\end{aligned}
$$

Where the boundary parameters of state element adopt upwind scheme: $\operatorname{Var}_{i}^{\text {in }}=\left\{\begin{array}{ll}\operatorname{Var}_{i-1} & \text { if } u_{i} \geq 0 \\ \operatorname{Var}_{i} & \text { if } u_{i}<0\end{array}, \operatorname{Var}_{-1}=\operatorname{Var}_{a}\right.$; $\operatorname{Var}_{i}^{\text {out }}=\left\{\begin{array}{ll}\operatorname{Var}_{i} & \text { if } u_{i+1} \geq 0 \\ \operatorname{Var}_{i+1} & \text { if } u_{i+1}<0\end{array}, \operatorname{Var}_{n}=\operatorname{Var}_{b} ; \operatorname{Var} \in\{\rho, p, E, \lambda, e\}\right.$. For the state element of liquid or low-velocity $(M a<0.3)$ gas, the effect of kinetic energy can be neglected, that is, $E_{i} \approx \rho_{i} e_{i}$.

a) For ideal gases, the internal energy state equation is introduced: $e_{i}=p_{i} /\left[\rho_{i}(\gamma-1)\right]$. As the gas specific heat ratio is assumed to be constant, the equation (4) can be:

$$
\begin{aligned}
\frac{\mathrm{d} p_{i}}{\mathrm{~d} t}=\frac{1}{V_{i}}\left[\gamma\left(p_{i}^{\text {in }} u_{i} A_{i}-p_{i}^{\text {out }} u_{i+1} A_{i+1}\right)+(\gamma-1)\left(\frac{1}{2} \rho_{i}^{\text {in }} u_{i}^{3} A_{i}-\frac{1}{2} \rho_{i}^{\text {out }} u_{i+1}^{3} A_{i+1}+\right.\right. \\
\left.\left.\quad \lambda_{i}^{\text {in }} \frac{T_{i-1}-T_{i}}{\bar{x}_{i}-\bar{x}_{i-1}} A_{i}-\lambda_{i}^{\text {out }} \frac{T_{i}-T_{i+1}}{\bar{x}_{i+1}-\bar{x}_{i}} A_{i+1}+\rho_{i} f_{x, i} V_{i} \frac{u_{i}+u_{i+1}}{2}-\dot{q}_{i} S_{i}\right)-\gamma p_{i} \frac{\mathrm{d} V_{i}}{\mathrm{~d} t}\right]
\end{aligned}
$$

b) For general compressible fluids, the internal energy state equation is introduced. Then the equation (4) can be:

$$
\begin{aligned}
\frac{\mathrm{d} p_{i}}{\mathrm{~d} t}= & \frac{1}{V_{i} e_{p, i}^{\prime}}\left[\left(\rho_{i}^{\text {in }} e_{i}^{\text {in }}+\frac{1}{2} \rho_{i}^{\text {in }} u_{i}^{2}+p_{i}^{\text {in }}\right) u_{i} A_{i}-\left(\rho_{i}^{\text {out }} e_{i}^{\text {out }}+\frac{1}{2} \rho_{i}^{\text {out }} u_{i+1}^{2}+p_{i}^{\text {out }}\right) u_{i+1} A_{i+1}+\right. \\
& \left.\lambda_{i}^{\text {in }} \frac{T_{i-1}-T_{i}}{\bar{x}_{i}-\bar{x}_{i-1}} A_{i}-\lambda_{i}^{\text {out }} \frac{T_{i}-T_{i+1}}{\bar{x}_{i+1}-\bar{x}_{i}} A_{i+1}+\rho_{i} f_{x, i} V_{i} \frac{u_{i}+u_{i+1}}{2}-\dot{q}_{i} S_{i}-\left(\rho_{i} e_{i}+p_{i}\right) \frac{\mathrm{d} V_{i}}{\mathrm{~d} t}\right]-\frac{e_{\rho, i}^{\prime}}{e_{p, i}^{\prime}} \frac{\mathrm{d} \rho_{i}}{\mathrm{~d} t}
\end{aligned}
$$

In the equation, $e_{p}^{\prime}=\rho \frac{\partial e}{\partial p}, e_{\rho}^{\prime}=e+\rho \frac{\partial e}{\partial \rho}$, and the internal energy state equation $e=e(p, \rho)$ can use a fitting formula based on physical property data.

2) For velocity element: $j=0,1, \cdots, n$

Given $W_{j}=u_{j}\left(\rho_{j-1} V_{j-1}+\rho_{j} V_{j}\right) / 2$, the momentum equation will be:

$$
\begin{aligned}
\mathrm{d} W_{j} / \mathrm{d} t= & \rho_{j-1}\left(u_{j}^{\text {in }}\right)^{2} \bar{A}_{j-1}-\rho_{j}\left(u_{j}^{\text {out }}\right)^{2} \bar{A}_{j}+\left(p_{j-1}-p_{j}\right) A_{j}+ \\
& \frac{\rho_{j-1} f_{x, j-1} V_{j-1}+\rho_{j} f_{x, j} V_{j}}{2}-f_{R, j} \frac{\rho_{j-1} V_{j-1}+\rho_{j} V_{j}}{2}, f_{x, j}=g \cos \theta_{j}, f_{R, j}=\frac{f_{\lambda, j} u_{j}\left|u_{j}\right|}{2 d_{j}}
\end{aligned}
$$

Where $\bar{A}_{-1}=\bar{A}_{a}, \bar{A}_{n}=\bar{A}_{b}, V_{-1}=\xi_{0} V_{0}, V_{n}=\xi_{n} V_{n-1}$; values of $\xi_{0}$ and $\xi_{n}$ can be $0 \sim 1$ based on the physical boundary conditions of pipe (taking the entrance closed pipe for example, $\xi_{0}=0$ ). When capturing sharp edge of shock wave, the upwind scheme is more precise than the arithmetic mean value for boundary velocities.

The flow in circular pipe can be divided into five regions according to Reynolds number: (1) Laminar region where $0 \leq R e \leq 2300$; (2) Transition region from laminar to turbulent flow where $2300<R e \leq 4000$; (3) Hydraulically smooth region where $4000<R e \leq 80 d / h_{\Delta}$; (4) Transition region from hydraulically smooth to rough where $80 d / h_{\Delta}<R e \leq 4160\left(d / 2 h_{\Delta}\right)^{0.85}$; (5) Hydraulically rough region where $R e>4160\left(d / 2 h_{\Delta}\right)^{0.85}$. Hagen-Poiseuille flow formula $f_{\lambda}=64 / R e$, Blasius formula, Blasius or Prandtl formula, Nikuradse or Colebrook-White formula, Karman-Nikuradse formula or other universal formulas [40-42] are adopted to calculate Darcy friction factor in abovementioned five regions, respectively.

3) Other equations: calculation formulas for pressure of state element and velocity of velocity element can be deduced from the definition formulas of total energy and momentum; calculation formula for temperature can be obtained from the state equation; after complementing the calculation formulas for sound velocity, heat flux density from fluid to wall and convective heat transfer coefficient of state element, the abovementioned equations become closed equations which can obtain numerical solution.

Taking the convective heat transfer coefficient for example, the flow in circular pipe can be divided into five regions: (1) $\operatorname{Re} \leq \sqrt{G r / 10}$; (2) $\sqrt{G r / 10}<\operatorname{Re} \leq 2300$; (3) $2300<\operatorname{Re} \leq 10^{4}$; (4) $10^{4}<\operatorname{Re} \leq 1.25 \times 10^{5}$ or 

formula, Dittus-Boelter formula, Sieder-Tate formula and so on [41-43] is selected according to pipe arrangement, fluid phase, temperature difference, cooling or heating condition, the values of $R e, \operatorname{Pr}$ and $R a$.

4) Determination of whole-field global time step for dynamic simulation

The local time step of state element determined based on the CFL (Courant-Friedrichs-Lewy) condition of 1D flow field is $\Delta t_{i}=C F L /\left[\frac{\left|\left(u_{i}+u_{i+1}\right) / 2\right|}{x_{i+1}-x_{i}}+\frac{a_{i}}{x_{i+1}-x_{i}}\right]$, where $0<C F L \leq 1, a$ is sound velocity.

During the dynamic simulation, the time-marching synchronization of the whole field (including flow field and pipe-wall temperature field) must be ensured. Therefore, the global time step is determined by:

$$
\Delta t=\min \left\{\min \left\{\Delta t_{i}\right\}, \min \left\{\Delta \tau_{i, k}\right\}\right\}
$$

\subsection{Finite volume model of temperature field in pipe wall}

As a natural extension of flow field modeling method, a finite volume model suitable for simulating pipe-wall transient heat transfer is obtained by employing two-dimensional finite control volume grids in axisymmetric cylindrical coordinates as shown in Fig. 1, which considers comprehensively the interact between pipe-wall temperature field and flow field and considers the influence of environment on system.

The unsteady state heat conduction differential equation, with any finite control volume in the pipe-wall temperature field, can work out the axisymmetric two-dimensional heat transfer model in the form of ordinary differential equations through spatial discretization.

For non-boundary elements: $i=0,1, \cdots, n-1, \quad k=1,2, \cdots, n_{w}-1$ and $k \neq k_{d}$

$$
\begin{gathered}
\frac{\mathrm{d} T_{i, k}}{\mathrm{~d} t}=\frac{1}{\rho c_{p}}\left\{\frac{\lambda_{\mathrm{w}}}{\Delta x \Delta x_{\mathrm{w}}} T_{i-1, k}+\frac{\lambda_{\mathrm{e}}}{\Delta x \Delta x_{\mathrm{e}}} T_{i+1, k}-\left[\frac{1}{\Delta x}\left(\frac{\lambda_{\mathrm{w}}}{\Delta x_{\mathrm{w}}}+\frac{\lambda_{\mathrm{e}}}{\Delta x_{\mathrm{e}}}\right)+\frac{1}{\Delta r}\left(\frac{\lambda_{\mathrm{s}}}{\Delta r_{\mathrm{s}}}+\frac{\lambda_{\mathrm{n}}}{\Delta r_{\mathrm{n}}}\right)\right] T_{i, k}+\right. \\
\left.\left(\frac{\lambda_{\mathrm{s}}}{\Delta r \Delta r_{\mathrm{s}}}-\frac{\lambda}{r_{i, k}\left(\Delta r_{\mathrm{s}}+\Delta r_{\mathrm{n}}\right)}\right) T_{i, k-1}+\left(\frac{\lambda_{\mathrm{n}}}{\Delta r \Delta r_{\mathrm{n}}}+\frac{\lambda}{r_{i, k}\left(\Delta r_{\mathrm{s}}+\Delta r_{\mathrm{n}}\right)}\right) T_{i, k+1}\right\}
\end{gathered}
$$

Heat conduction equations which slightly differ from equation (9) can be obtained at convective heat transfer internal boundary $(k=0)$, convective or radiation heat transfer external boundary $\left(k=n_{w}\right)$, and interface of two materials $\left(k=k_{d}\right)$. The difference form of heat transfer model can be obtained by discretizing the above equations. Then, according to the second law of thermodynamics, the stability criterion of solving the model can be obtained:

$$
\Delta \tau_{i, k} \leq \rho c_{p} /\left[\frac{1}{\Delta x}\left(\frac{\lambda_{\mathrm{w}}}{\Delta x_{\mathrm{w}}}+\frac{\lambda_{\mathrm{e}}}{\Delta x_{\mathrm{e}}}\right)+\frac{1}{\Delta r}\left(\frac{\lambda_{\mathrm{s}}}{\Delta r_{\mathrm{s}}}+\frac{\lambda_{\mathrm{n}}}{\Delta r_{\mathrm{n}}}\right)\right], i=0,1, \cdots, n-1, k=1,2, \cdots, n_{w}-1 \text { and } k \neq k_{d}
$$

Under conditions of aforementioned three heat transfer internal and external boundaries and interface of two materials, four specific expressions of the stability criterion can be obtained. In the case of uniform-distribution grids and constant physical property, $\operatorname{RET}\left\{\Delta \tau_{i, k}\right\}>\min \left\{\operatorname{RET}\left\{\Delta \tau_{i, 0}\right\}, \operatorname{RET}\left\{\Delta \tau_{i, n_{w}}\right\}\right\}$, where $\operatorname{RET}\{\Delta \tau\}$ is the right-end term of each stability criterion expression. If it is still assumed to be true in the case of non-uniform grids and variable physical property, the time step for calculating pipe-wall heat transfer can be calculated by:

$$
\Delta \tau=\min \left\{\Delta \tau_{i, 0}, \Delta \tau_{i, n_{w}}, \Delta \tau_{i, k_{d}}\right\} / 1.2, i=0,1, \cdots, n-1
$$

In fact, the simulation research shows that the temperature-field time step determined by the stability criterion is usually greater than the flow-field time step $\Delta t$ in transient pipe flow, so $\Delta \tau=\Delta t$ can be applicable for the dynamic simulation and the above temperature-field criterion can be used as a verification condition.

In the equations, $r_{k}=r_{0}+\sum_{j=0}^{k-1} \Delta r_{j}+\frac{\Delta r_{k}}{2}, k=1,2, \cdots, n_{w}-1$. Note that $\Delta r_{0}$ and $\Delta r_{n_{*}}$ are half of general grid radial length. The heat conductivity coefficient at the boundary of each grid element adopts the harmonic average value of heat conductivity coefficients of two grid elements located on both sides of the boundary. $T_{-1, k}$ and $T_{n, k}$ are component-boundary wall temperatures, values of which can be determined by boundary wall grids of upstream and downstream components or also simply calculated by $T_{-1, k}=T_{0, k}, T_{n, k}=T_{n-1, k}$.

Although the $2 \mathrm{D}$ heat transfer model of pipe wall is more accurate and comprehensive, the coupled iterative calculation of temperature filed and flow field would inevitably decelerate seriously the distributed parameter system dynamic simulation. Thereby, the 1D heat transfer model along radial direction can be employed on 
condition that the temperature gradient of fluid along pipe axial direction is relatively small, the 0D heat transfer model or adiabatic model can also be employed on condition that the temperature difference among fluid, wall and environment is relatively small or the insulation measures are very good.

\subsection{Modularization algorithm of specific components}

During establishing the model of a system, conventional modeling method usually correlates system structure with computer program, which determines that the program codes must be modified if the system structure changes. The modularization method [38] has solved the problem effectively. Table 1 shows the module information of six typical components after modularization disassembly of double-valve combined test system, in which fluid source, gas pipe, gas volume and gas valve are four basic modules, and gas pressure reducing regulator (GPRR) and check valve are two modules obtained by combinative development from some basic modules.

The fluid source module sets up three types of inlet fluid sources and three types of outlet fluid sources which are designated to provide other components with inlet and outlet boundary conditions. The model of gas pipe module contains Equations (3), (4), (7) and ideal-gas state equation. Below are the models of the other four modules.

\subsubsection{Gas volume module}

Fig. 2 shows the gas volume with three inlets and three outlets. Its boundary is made up of half a velocity element extended from each gas pipe boundary. In the gas volume state element, the state parameters such as pressure and density are instantaneously consistent and uniform. When the frictional and local losses and the effects of axial heat conduction and gravity field are neglected, Equations (3) and (5) can generate the following model equations:

$$
\begin{aligned}
& \text { Continuity equation: } \frac{\mathrm{d} \rho}{\mathrm{d} t}=\frac{1}{V}\left[\sum_{i=1}^{n_{\text {in }}} \rho_{i}^{\text {in }}\left(u_{n} A_{n}\right)_{i}-\sum_{j=1}^{n_{\text {out }}} \rho_{j}^{\text {out }}\left(u_{0} A_{0}\right)_{j}\right]-\frac{\rho}{V} \frac{\mathrm{d} V}{\mathrm{~d} t} \\
& \frac{\mathrm{d} p}{\mathrm{~d} t}=\frac{1}{V}\left\{\sum_{i=1}^{n_{\text {in }}}\left[\gamma p_{i}^{\text {in }}\left(u_{n} A_{n}\right)_{i}+\frac{\gamma-1}{2} \rho_{i}^{\text {in }}\left(u_{n}^{3} A_{n}\right)_{i}\right]-\right. \\
& \text { Energy equation: } \\
& \left.\sum_{j=1}^{n_{\text {out }}}\left[\gamma p_{j}^{\text {out }}\left(u_{0} A_{0}\right)_{j}+\frac{\gamma-1}{2} \rho_{j}^{\text {out }}\left(u_{0}^{3} A_{0}\right)_{j}\right]-(\gamma-1) \dot{q} S-\gamma p \frac{\mathrm{d} V}{\mathrm{~d} t}\right\}
\end{aligned}
$$

For low-velocity $(M a<0.3)$ gases, when further ignoring the effects of inlet and outlet kinetic energy, the energy

equation can be simplified as: $\frac{\mathrm{d} p}{\mathrm{~d} t}=\frac{\gamma}{V}\left[\sum_{i=1}^{n_{\text {in }}} p_{i}^{\text {in }}\left(u_{n} A_{n}\right)_{i}-\sum_{j=1}^{n_{\text {out }}} p_{j}^{\text {out }}\left(u_{0} A_{0}\right)_{j}-p \frac{\mathrm{d} V}{\mathrm{~d} t}\right]-\frac{\gamma-1}{V} \dot{q} S$

The numbers of inlets and outlets are determined by values of the variables $n_{\text {in }}$ and $n_{\text {out }}$, respectively, by which the modularization of any gas volumes with the same subprogram is realized. When calculating mass exchange and energy exchange between the state element of gas volume and connecting pipes, the upwind scheme is used to ensure compatibility of the equations. The adiabatic or 0D wall heat transfer models can be chosen.

\subsubsection{Gas valve module}

Components which have small length-diameter ratio and abrupt change of cross section in fluid flow direction, such as filter, stop valve, ball valve, compensator, feed/discharge valve, safety valve, orifice plate and so on, can be considered to be formed by connecting two gas volume elements with local flow resistance. Their models contain finite volume models of upstream and downstream gas volume elements, valve spool and orifice throttling models and flow coefficient calculation schemes. The throttling models include pressure ratio-based and pressure difference-based injector orifice models [38] and a dual-model solution scheme [37], which are used to describe the throttling role and the opening/closing characteristics of valve spool. Eight calculation schemes of flow coefficient are set up and a scheme-controller variable is set up for users to choose an appropriate solution [37]. The concrete form of gas-valve-module model will be reflected somewhat in model of CV module and is not repeated herein.

\subsubsection{Check valve module}

Fig. 3 shows the schematic of the double-spool lift CV used in the integral bipropellant propulsion system. The opening and flow characteristics of the $\mathrm{CV}$ are decided by sizes and geometric shapes of the flow sections between big or small valve spool and their own valve seats as well as the non-return orifice. Its working principle is: when the CV upstream and downstream pressure difference is higher than the opening pressure difference, the valve spools open and then the gas flows to the downstream; otherwise, the opening force acting on spool moving parts is weaker than the closing force of the springs and the spools close.

Fig. 4 shows the finite control volume grids of the CV. At the boundaries, there are boundary grids of connecting gas pipes. The $\mathrm{CV}$ is regarded to be made up of four gas volumes including high- and low-pressure chambers, 
non-return and opening chambers, which are connected by local flow resistances. Volumes of four chambers vary significantly with opening and closing of the spools and need to be considered as variable-volume gas volumes.

The basic idea of mathematical model derivation: the state parameters which are difficult to deal with in the gas volume model are its velocity terms. For a chamber with multiple inlets and outlets, the fluid in the chamber does not have a uniform velocity with clear physical meaning and certainly flows by zone. Therefore, the derivation uses the physical variables with relatively clear physical meaning such as pressure, density, flow rate at throttling point, inlet and outlet flow rates to replace the velocity terms. According to the modeling idea, since the $\mathrm{CV}$ is considered as a combinational component of gas volume and local flow resistance, its model should be a natural extension of combinational application of gas volume and gas valve models as follows.

1) High- and low-pressure Chambers

Continuity equations: $\left\{\begin{array}{l}\frac{\mathrm{d} \rho_{1}}{\mathrm{~d} t}=\frac{1}{V_{1}}\left(\rho_{1}^{\text {in }} u_{n} A_{n}-Q_{\mathrm{m} 1}-\rho_{1} \frac{\mathrm{d} V_{1}}{\mathrm{~d} t}\right) \\ \frac{\mathrm{d} \rho_{2}}{\mathrm{~d} t}=\frac{1}{V_{2}}\left(Q_{\mathrm{m} 3}-\rho_{20}^{\text {out }} u_{0} A_{0}-\rho_{2} \frac{\mathrm{d} V_{2}}{\mathrm{~d} t}\right)\end{array}\right.$
Energy equations: $\left\{\begin{array}{l}\frac{\mathrm{d} p_{1}}{\mathrm{~d} t}=\frac{\gamma}{V_{1}}\left(p_{1}^{\text {in }} u_{n} A_{n}-p_{1}^{\text {out }} \frac{Q_{\mathrm{m} 1}}{\rho_{1}^{\text {out }}}-p_{1} \frac{\mathrm{d} V_{1}}{\mathrm{~d} t}\right)-\frac{\gamma-1}{V_{1}} \dot{q}_{1} S_{1} \\ \frac{\mathrm{d} p_{2}}{\mathrm{~d} t}=\frac{\gamma}{V_{2}}\left(p_{2}^{\text {in }} \frac{Q_{\mathrm{m} 3}}{\rho_{2}^{\text {in }}}-p_{20}^{\text {out }} u_{0} A_{0}-p_{2} \frac{\mathrm{d} V_{2}}{\mathrm{~d} t}\right)-\frac{\gamma-1}{V_{2}} \dot{q}_{2} S_{2}\end{array}\right.$

Temperature calculation formulas: $T_{1}=p_{1} /\left(\rho_{1} R\right), T_{2}=p_{2} /\left(\rho_{2} R\right)$

2) Big valve spool throttling point

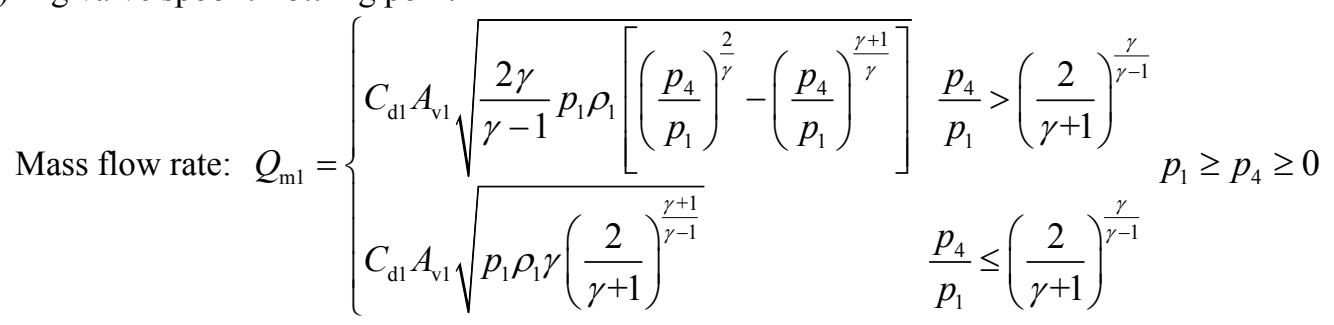

Based on the schematic of big valve spool throttling as shown in Fig. 5, the sectional flow area in the case of the spool opening being $h_{1}$ can be obtained: $A_{\mathrm{v} 1}=2 \pi h_{1} b_{1}\left(1+2 b_{1} h_{1} / d \mathrm{c}_{2}{ }^{2}\right) d_{\mathrm{v} 1} / d \mathrm{c}_{2}$

High-pressure chamber volume: $V_{1}=V_{1}^{\prime}+\frac{\left(r_{\mathrm{v} 1}+h_{1} \sin \alpha_{1} \cos \alpha_{1}\right)^{3}-r_{\mathrm{v} 1}^{3}}{3 \sin \alpha_{1} \cos \alpha_{1}} \pi, V_{1}^{\prime}=V_{\text {pipe } 1}+V_{1 \text { cone }}^{\prime}$

High-pressure chamber volume derivative: $\frac{\mathrm{d} V_{1}}{\mathrm{~d} t}=\pi\left(r_{\mathrm{v} 1}+h_{1} \sin \alpha_{1} \cos \alpha_{1}\right)^{2} \frac{\mathrm{d} h_{1}}{\mathrm{~d} t}$

where $\quad b_{1}=\sqrt{\left(d \mathrm{c}_{2} / 2\right)^{2}-\left(d_{\mathrm{v} 1} / 2\right)^{2}} \quad, \quad \sin \alpha_{1}=\sqrt{d \mathrm{c}_{2}{ }^{2}-d_{\mathrm{v} 1}^{2}} / d \mathrm{c}_{2} \quad, \quad \cos \alpha_{1}=d_{\mathrm{v} 1} / d \mathrm{c}_{2} \quad, \quad V_{\text {pipel }}=\pi r_{1}^{2} l_{1} \quad$, $V_{1 \text { cone }}^{\prime}=\frac{r_{\mathrm{v} 1}^{3}-r_{1}^{3}}{3} \pi \cdot \cot \alpha_{1}-\pi \frac{2 r \mathrm{c}_{2}{ }^{3}-3 r \mathrm{c}_{2}{ }^{2} \sqrt{r \mathrm{c}_{2}{ }^{2}-r_{\mathrm{t} 2}{ }^{2}}+\left(r \mathrm{c}_{2}{ }^{2}-r_{\mathrm{t} 2}{ }^{2}\right)^{3 / 2}}{3}, r_{\mathrm{t} 2}=r_{\mathrm{v} 1}=\frac{d_{\mathrm{v} 1}}{2} ; V_{1}$ and $V_{1}{ }^{\prime}$ are volumes of high-pressure chamber in the case of big-spool opening being $h_{1}$ and $h_{1}=0$, respectively; $V_{\text {pipe } 1}$ and $V_{1 \text { cone }}^{\prime}$ are volumes of the inlet straight pipe section and the conical part of high-pressure chamber in the case of $h_{1}=0$, respectively; $d \mathrm{c}_{2}$ is diameter of the sphere part of big spool; $d_{\mathrm{v} 1}$ is diameter of the circular part of big spool in contact with conical valve seat at fully closing position $\left(h_{1}=0\right) ; d_{1}, r_{1}$ and $l_{1}$ are diameter, radius and length of the inlet straight pipe section of high-pressure chamber, respectively; Flow coefficient $C_{\mathrm{d} 1}$ represents spool throttling characteristic, and its value is the function of $h_{1}$. The $C_{\mathrm{d} 1} \sim h_{1}$ curves need to be established for different kinds of valve spools by test and appropriately amended for different fluid media according to viscosity and density.

Local flow velocity calculation formula of specific section: $u_{\mathrm{A}}=Q_{\mathrm{m} 1} /\left(\rho_{\mathrm{A}} A_{\mathrm{A}}\right)$ 
3) Small valve spool throttling point

Mass flow rate: $Q_{\mathrm{m} 3}= \begin{cases}C_{\mathrm{d} 3} A_{\mathrm{v} 3} \sqrt{\frac{2 \gamma}{\gamma-1} p_{3} \rho_{3}\left[\left(\frac{p_{2}}{p_{3}}\right)^{\frac{2}{\gamma}}-\left(\frac{p_{2}}{p_{3}}\right)^{\frac{\gamma+1}{\gamma}}\right]} & \frac{p_{2}}{p_{3}}>\left(\frac{2}{\gamma+1}\right)^{\frac{\gamma}{\gamma-1}} \\ C_{\mathrm{d} 3} A_{\mathrm{v} 3} \sqrt{p_{3} \rho_{3} \gamma\left(\frac{2}{\gamma+1}\right)^{\frac{\gamma+1}{\gamma-1}}} & \frac{p_{2}}{p_{3}} \leq\left(\frac{2}{\gamma+1}\right)^{\frac{\gamma}{\gamma-1}}\end{cases}$

The sectional flow area when small spool opening is $h_{2}: A_{\mathrm{v} 3}=2 \pi h_{2} b_{2}\left(1+2 b_{2} h_{2} / d \mathrm{c}_{7}{ }^{2}\right) d_{\mathrm{v} 3} / d \mathrm{c}_{7}$

Low-pressure chamber volume: $V_{2}=V_{2}^{\prime}-\frac{\left(r_{\mathrm{v} 3}+h_{2} \sin \alpha_{2} \cos \alpha_{2}\right)^{3}-r_{\mathrm{v} 3}{ }^{3}}{3 \sin \alpha_{2} \cos \alpha_{2}} \pi$

Low-pressure chamber volume derivative: $\frac{\mathrm{d} V_{2}}{\mathrm{~d} t}=-\pi\left(r_{\mathrm{v} 3}+h_{2} \sin \alpha_{2} \cos \alpha_{2}\right)^{2} \frac{\mathrm{d} h_{2}}{\mathrm{~d} t}$

In the equations, the physical meanings of various variables are similar to those of big valve spool.

4) Opening chamber

Continuity equation: $\frac{\mathrm{d} \rho_{4}}{\mathrm{~d} t}=\frac{1}{V_{4}}\left(Q_{\mathrm{m} 1}-Q_{\mathrm{m} 4}-\rho_{4} \frac{\mathrm{d} V_{4}}{\mathrm{~d} t}\right)$

Energy equation: $\frac{\mathrm{d} p_{4}}{\mathrm{~d} t}=\frac{\gamma}{V_{4}}\left(p_{4}^{\text {in }} \frac{Q_{\mathrm{m} 1}}{\rho_{4}^{\text {in }}}-p_{4}^{\text {out }} \frac{Q_{\mathrm{m} 4}}{\rho_{4}^{\text {out }}}-p_{4} \frac{\mathrm{d} V_{4}}{\mathrm{~d} t}\right)-\frac{\gamma-1}{V_{4}} \dot{q}_{4} S_{4}$

Opening chamber volume: $V_{4}=V_{4}^{\prime}+\frac{\pi}{4} d_{4}{ }^{2} h_{1}-\frac{\left(r_{\mathrm{v} 1}+h_{1} \sin \alpha_{1} \cos \alpha_{1}\right)^{3}-r_{\mathrm{v} 1}{ }^{3}}{3 \sin \alpha_{1} \cos \alpha_{1}} \pi$

Opening chamber volume derivative: $\frac{\mathrm{d} V_{4}}{\mathrm{~d} t}=\pi\left[\frac{d_{4}{ }^{2}}{4}-\left(r_{\mathrm{v} 1}+h_{1} \sin \alpha_{1} \cos \alpha_{1}\right)^{2}\right] \frac{\mathrm{d} h_{1}}{\mathrm{~d} t}$

In the equations, $V_{4}$ and $V_{4}{ }^{\prime}$ are volumes of opening chamber in the case of big valve spool opening being $h_{1}$ and $h_{1}=0$, respectively; $d_{4}$ is internal diameter of opening chamber.

5) Non-return orifice throttling point

Mass flow rate: $Q_{\mathrm{m} 4}= \begin{cases}C_{\mathrm{d} 4} A_{\mathrm{v} 4} \sqrt{\frac{2 \gamma}{\gamma-1} p_{4} \rho_{4}\left[\left(\frac{p_{3}}{p_{4}}\right)^{\frac{2}{\gamma}}-\left(\frac{p_{3}}{p_{4}}\right)^{\frac{\gamma+1}{\gamma}}\right]} & \frac{p_{3}}{p_{4}}>\left(\frac{2}{\gamma+1}\right)^{\frac{\gamma}{\gamma-1}} \\ C_{\mathrm{d} 4} A_{\mathrm{v} 4} \sqrt{p_{4} \rho_{4} \gamma\left(\frac{2}{\gamma+1}\right)^{\frac{\gamma+1}{\gamma-1}}} & \frac{p_{3}}{p_{4}} \leq\left(\frac{2}{\gamma+1}\right)^{\frac{\gamma}{\gamma-1}}\end{cases}$

Where non-return orifice flow area $A_{\mathrm{v} 4}=\pi d_{\mathrm{v} 4}^{2} / 4$. In the case of reverse flow, that is, when the equation meets $0 \leq p_{4}<p_{3}$, the positions of $p_{4}$ and $p_{3}$ are exchanged and a minus sign is added in front of the mass flow rate result.

6) Non-return chamber

Continuity equation: $\frac{\mathrm{d} \rho_{3}}{\mathrm{~d} t}=\frac{1}{V_{3}}\left(Q_{\mathrm{m} 4}-Q_{\mathrm{m} 3}-\rho_{3} \frac{\mathrm{d} V_{3}}{\mathrm{~d} t}\right)$

Energy equation: $\frac{\mathrm{d} p_{3}}{\mathrm{~d} t}=\frac{\gamma}{V_{3}}\left(p_{3}^{\text {in }} \frac{Q_{\mathrm{m} 4}}{\rho_{3}^{\text {in }}}-p_{3}^{\text {out }} \frac{Q_{\mathrm{m} 3}}{\rho_{3}^{\text {out }}}-p_{3} \frac{\mathrm{d} V_{3}}{\mathrm{~d} t}\right)-\frac{\gamma-1}{V_{3}} \dot{q}_{3} S_{3}$

Non-return chamber volume: $V_{3}=V_{3}^{\prime}-\frac{\pi}{4} d_{3}{ }^{2} h_{1}+\frac{\left(r_{\mathrm{v} 3}+h_{2} \sin \alpha_{2} \cos \alpha_{2}\right)^{3}-r_{\mathrm{v} 3}{ }^{3}}{3 \sin \alpha_{2} \cos \alpha_{2}} \pi$

Non-return chamber volume derivative: $\frac{\mathrm{d} V_{3}}{\mathrm{~d} t}=\pi\left[\left(r_{\mathrm{v} 3}+h_{2} \sin \alpha_{2} \cos \alpha_{2}\right)^{2} \frac{\mathrm{d} h_{2}}{\mathrm{~d} t}-\frac{d_{3}^{2}}{4} \frac{\mathrm{d} h_{1}}{\mathrm{~d} t}\right]$

In the equations, $V_{3}^{\prime}{ }_{\text {cone }}=\frac{r_{\mathrm{v} 3}{ }^{3}-r \mathrm{c}_{5}{ }^{3}}{3} \pi \cdot \cot \alpha_{2}-\pi \frac{2 r \mathrm{c}_{7}{ }^{3}-3 r \mathrm{c}_{7}{ }^{2} \sqrt{r \mathrm{c}_{7}{ }^{2}-r_{\mathrm{v} 3}{ }^{2}}+\left(r \mathrm{c}_{7}{ }^{2}-r_{\mathrm{v} 3}{ }^{2}\right)^{3 / 2}}{3} ; V_{3}$ and $V_{3}{ }^{\prime}$ are 
$\cdot 10$.

volumes of non-return chamber in the case of big-spool opening being $h_{1}$, small-spool opening being $h_{2}$ and $h_{1}=h_{2}=0$, respectively; $V_{3}^{\prime}$ cone is volume of the conical part of non-return chamber in the case of $h_{1}=h_{2}=0 ; d_{3}$ is internal diameter of non-return chamber; $d \mathrm{c}_{5}$ is inlet diameter of the conical part of non-return chamber.

7) Force equilibrium equations

Movements of big and small valve spools are restricted by the valve seats in front of and behind them. In addition, there is collision problem when big and small spools contact. Therefore, there is involvement of collision dynamics issue with multi-constraint conditions. The modeling uses the assumptions for collision issue as follows:

a) The collision between valve spool and valve seat in front of or behind it is completely inelastic collision, and the momentum of valve spool instantaneously reduces to zero when colliding with the valve seat, that is, at the moment of collision, $u_{\mathrm{VC}}=0$ or $\mathrm{d} h / \mathrm{d} t=0$;

b) The collision between big and small spools occurs only at the moment from the state of separation to the state of contact and then they enter the state of adhesion immediately after collision. Only some certain conditions (such as $h_{1}-h_{2}<h_{\max 3}$ ) can transfer them into the state of separation;

c) The collision between two valve spools is completely inelastic collision, and they instantaneously adhere to each other after contact, that is, at the moment of collision, $u_{\mathrm{VC} 1}=u_{\mathrm{VC} 2}, a_{\mathrm{VC} 1}=a_{\mathrm{VC} 2}$ or $\mathrm{d} h_{1} / \mathrm{d} t=\mathrm{d} h_{2} / \mathrm{d} t$, $\mathrm{d}^{2} h_{1} / \mathrm{d} t^{2}=\mathrm{d}^{2} h_{2} / \mathrm{d} t^{2}$.

It can be seen from Fig. 3: when $h_{1}-h_{2}<h_{\max 3}$, it is impossible for big and small spools to contact and the movements of two spools are only restricted by their own front and rear valve seats; when $h_{1}-h_{2} \geq h_{\max 3}$, two spools are not only restricted by valve seats but also possible to collide with each other. Therefore, it is very complicated to deal with the logic of spool movement, and various conditions arising out of combination of different factors need to be taken into consideration. Below only gives several typical conditions in the solution.

a) In the case of free movement of the big spool and the small spool without restriction:

$$
\begin{aligned}
& \left\{\begin{array}{l}
\mathrm{d} h_{1} / \mathrm{d} t=h_{1 \mathrm{~d}}, \quad 0 \leq h_{1} \leq h_{\max 1} \\
\frac{\mathrm{d} h_{\mathrm{ld}}}{\mathrm{d} t}=a_{\mathrm{vC} 1}=\left[-C_{1}\left(x_{1}+h_{1}\right)+p_{1} A_{1}^{\prime}+p_{4} A_{4}^{\prime}-p_{3} A_{31}{ }^{\prime}-\beta_{1} h_{\mathrm{ld}}\right] / m_{\mathrm{vC} 1}
\end{array}\right. \\
& \left\{\begin{array}{l}
\mathrm{d} h_{2} / \mathrm{d} t=h_{2 \mathrm{~d}}, \quad 0 \leq h_{2} \leq h_{\max 2} \\
\frac{\mathrm{d} h_{2 \mathrm{~d}}}{\mathrm{~d} t}=a_{\mathrm{vC} 2}=\left[-C_{2}\left(x_{2}+h_{2}\right)+p_{3} A_{32}{ }^{\prime}-p_{2} A_{2}{ }^{\prime}-\beta_{2} h_{2 \mathrm{~d}}\right] / m_{\mathrm{vC} 2}
\end{array}\right.
\end{aligned}
$$

b) When two valve spools adhesively move together:

According to specific situation, there is $h_{1}=h_{2}+h_{\max 3}$ or $h_{2}=h_{1}-h_{\max 3}$

$$
\left\{\begin{array}{l}
\mathrm{d} h_{1} / \mathrm{d} t=h_{1 \mathrm{~d}}, \quad 0 \leq h_{1} \leq h_{\max 1} ; \quad \mathrm{d} h_{2} / \mathrm{d} t=h_{2 \mathrm{~d}}, \quad 0 \leq h_{2} \leq h_{\max 2} \\
\frac{\mathrm{d} h_{1 \mathrm{~d}}}{\mathrm{~d} t}=\frac{\mathrm{d} h_{2 \mathrm{~d}}}{\mathrm{~d} t}=\frac{-C_{1}\left(x_{1}+h_{1}\right)-C_{2}\left(x_{2}+h_{2}\right)+p_{1} A_{1}^{\prime}+p_{4} A_{4}^{\prime}+p_{3}\left(A_{32}^{\prime}-A_{31}^{\prime}\right)-p_{2} A_{2}^{\prime}-\left(\beta_{1}+\beta_{2}\right) h_{1 \mathrm{~d}}}{m_{\mathrm{vC} 1}+m_{\mathrm{vC} 2}}
\end{array}\right.
$$

c) When two valve spools adhesively move together after contact and collision:

At the moment of collision, the momentum conservation equation can derive:

$h_{1 \mathrm{~d}}=h_{2 \mathrm{~d}}=\left(m_{\mathrm{VC} 1} h_{1 \mathrm{~d}}^{\text {BeforeHit }}+m_{\mathrm{VC} 2} h_{2 \mathrm{~d}}^{\text {BeforeHit }}\right) /\left(m_{\mathrm{VC} 1}+m_{\mathrm{VC} 2}\right)$

According to specific situation, there is $h_{1}=h_{2}+h_{\max 3}$ or $h_{2}=h_{1}-h_{\max 3}$

d) When big or small spool collides with the valve seat in front of it:

At the moment of collision, $h_{i}=0, \mathrm{~d} h_{i} / \mathrm{d} t=h_{i \mathrm{~d}}=0, i=1,2$

e) When big or small spool collides with the valve seat behind it:

At the moment of collision, $h_{i}=h_{\max i}, \mathrm{~d} h_{i} / \mathrm{d} t=h_{i \mathrm{~d}}=0, i=1,2$

The damping coefficient of big or small valve spool movement: $\beta_{i}=\lambda_{\mathrm{s} i} \cdot C_{i}$, where $\lambda_{\mathrm{s} i}=(1.5 \sim 4) \times 10^{-4} \mathrm{~s}, i=1,2$

In above equations, $C_{1}$ and $C_{2}$ are stiffnesses of big spring and small spring, respectively; $x_{1}$ and $x_{2}$ are pre-compressed spring lengths when valve spools are closed completely $\left(h_{1}=0, h_{2}=0\right) ; A_{1}{ }^{\prime}, A_{4}{ }^{\prime}$ and $A_{31}{ }^{\prime}$ are effective areas of high-pressure-chamber, opening-chamber and non-return-chamber gases acting on big spool, respectively; $A_{2}{ }^{\prime}$ and $A_{32}{ }^{\prime}$ are effective areas of low-pressure-chamber and non-return-chamber gases acting on small spool, respectively; $m_{\mathrm{VC} 1}$ and $m_{\mathrm{VC} 2}$ are masses of big and small spools, respectively; $u_{\mathrm{VC} 1}, u_{\mathrm{VC} 2}$ and $a_{\mathrm{VC} 1}, a_{\mathrm{VC} 2}$ are velocities and accelerations of big and small spools, respectively, and their positive direction is assumed to be the opening direction of two valve spools; $h_{\max 1}$ and $h_{\max 2}$ are the maximum openings of big and small spools, 
respectively; $h_{\max 3}$ is the separation distance between big and small spools when being closed completely.

8) Wall heat transfer model

One of the radial 1D heat transfer model, the 0D heat transfer model and the adiabatic model can be chosen in line with the actual condition of temperature field.

\subsubsection{Gas pressure reducing regulator module}

The PRR, a component working by the throttling principle, is to reduce pressure of inlet high-pressure fluid to the defined value and keep it within a certain scope. From the perspective of fluid dynamics, the PRR is a throttling component with variable local resistance, which reduces pressure by changing the throttling area to turn the fluid flow work into the kinetic energy and then to the intermolecular potential energy so as to generate different pressure losses. Meanwhile, by adjustment of control and adjusting systems, the acting force of downstream pressure on the regulator is kept in dynamic equilibrium with the forces of elastic components (and fluid pressure of control chamber for certain types of regulators) to adapt to fluctuations in pressure or flow rate, and finally the pressure behind regulator is maintained constant within a certain error range. The PRR of the integral bipropellant propulsion system is a high-pressure-unloading diaphragm-type regulator and its schematic is shown in Fig. 6.

Fig. 7 shows the finite control volume grids of the regulator. The PRR is regarded to be made up of four gas volumes. Because the valve-spool diameter is much less than the diaphragm diameter, the volumes of high- and low-pressure chambers do not change obviously with opening and closing of the valve spool and they can be viewed as constant-volume gas volumes. The volumes of feedback and unloading chambers vary significantly with valve spool changing and need to be considered as variable-volume gas volumes.

The basic idea of the mathematical model derivation is the same as that of the CV. Below only gives the flow area calculation formulas of valve spool throttling point and the force equilibrium equation.

Based on the schematic of valve spool throttling of the PRR as shown in Fig. 8, the sectional flow area in the case of the spool opening being $h$ can be obtained: $A_{\mathrm{v} 1}=\pi d_{\mathrm{v} 1} a h(1+h / 2 a) / \sqrt{\left(d_{\mathrm{v} 1} / 2\right)^{2}+(a+h)^{2}}$

Where $a=\sqrt{\left(d \mathrm{c}_{1} / 2\right)^{2}-\left(d_{\mathrm{v} 1} / 2\right)^{2}} ; d \mathrm{c}_{1}$ and $d_{\mathrm{v} 1}$ are diameters of spool steel ball and valve seat, respectively.

In order to use the Runge-Kutta method to solve the model, the force equilibrium equation in the form of second-order differential equation is rewritten as:

$$
\left\{\begin{array}{l}
\mathrm{d} h / \mathrm{d} t=h_{\mathrm{d}}, 0 \leq h \leq h_{\max } \\
\frac{\mathrm{d} h_{\mathrm{d}}}{\mathrm{d} t}=\left[C_{\mathrm{m}} x_{0}+C_{2} x_{2}+C_{3} x_{3}-C_{1} x_{1}-C_{\Sigma} h+p_{\mathrm{c}} A_{\mathrm{m}}^{\prime}+p_{1}\left(A_{0}^{\prime}-A_{1}^{\prime}\right)-p_{2} A_{2}^{\prime}-p_{3} A_{\mathrm{m}}^{\prime}-p_{4} A_{4}^{\prime}-\beta h_{\mathrm{d}}\right] / m_{\mathrm{VC}}
\end{array}\right.
$$

Effective area of diaphragm $A_{\mathrm{m}}{ }^{\prime}=\frac{1}{3}\left[1+d_{\mathrm{g}} / d_{\mathrm{m}}+\left(d_{\mathrm{g}} / d_{\mathrm{m}}\right)^{2}\right] \pi d_{\mathrm{m}}{ }^{2} / 4$

The damping coefficient of valve spool movement $\beta=\lambda_{\mathrm{s}} \cdot C_{\Sigma}$, where $\lambda_{\mathrm{s}}=(1.5 \sim 4) \times 10^{-4} \mathrm{~s}, C_{\Sigma}=C_{\mathrm{m}}+C_{1}+C_{2}+C_{3}$

Where $p_{\mathrm{c}}$ is pressure of atmosphere chamber; $A_{0}{ }^{\prime}$ and $A_{1}{ }^{\prime}$ are effective areas of downward and upward acting of high-pressure-chamber gas on the spool; $d_{\mathrm{g}}$ and $d_{\mathrm{m}}$ are diameters of rigid plate and diaphragm, respectively.

\subsection{Verification of codes}

The validity and generality of the model system have been verified via applications in some engineering systems including a turbine test rig system [37,38], several types of pressure-reducing-regulator systems [39,44], a liquid oxygen tank pressurization system [45], etc. The errors between most dynamic simulation curves and experimental or experiential curves are within $5 \%$. Taking the turbine test rig system for example, the 260 s dynamic simulation gets the results completely consistent with the experiment in combustion-gas temperature of the heater (combustion device); the simulation curves of other sensor measuring points behind the heater describe the temperature stratification phenomenon with the maximum error of 5\% compared with the experimental dynamic measurement curves for wall temperature and the maximum error of $20 \%$ for flow-field temperature.

The verifications of its applicability and accuracy in two classic problems are given below.

\subsubsection{One-dimensional Sod shock tube problem}

The 1D Riemann problem used by Sod [46,47] is modeled by a 4m-long gas pipe (GP1) as shown in Fig. 9(a). Fig. 10(a) gives the pressure, density and velocity profiles along the pipe axis at time instant $1 \mathrm{~ms}$. It can be seen from the comparison of adiabatic-wall simulation results and analytical solution that numerical results match well with exact solution. The propagating shock wave and the contact discontinuity are captured well without significant spurious oscillations. 


\subsubsection{Venting air tank problem}

Majumdar [48] use a model with two nodes and one branch to simulate the blown-down process of a pressurized tank. Comparison of simulation results with isentropic analytical solution verifies the effectiveness of the Generalized Fluid System Simulation Program (GFSSP) [49]. The venting air tank problem is modeled here by a modularization system composed of 1 gas volume (GVol1), 1 gas injector (GI1) and 1 fluid source (FS1) as shown in Fig. 9(b). The model of gas injector (injection cavity + orifice) is similar with that of gas valve. The outlet-fluid-source boundary condition for FS1 is constant-pressure atmospheric environment.

Fig. 10(b) gives the pressure and temperature history of air tank. The comparison of adiabatic-wall simulation results with isentropic analytical solution shows that 0-200s numerical curves are well consistent with the analytical solution. The simulation pressure and temperature of GVoll are $0.269230 \mathrm{MPa}$ and $229.180 \mathrm{~K}$ at 200 s, respectively while the values of analytical solution are $0.269228 \mathrm{MPa}$ and $229.177 \mathrm{~K}$.

\section{Numerical modeling of the system}

\subsection{System model}

The numerical model of double-valve combined test system is shown in Fig. 11, in which length (unit: m), outer diameter and thickness (unit: $\mathrm{mm}$ ) of every pipe, volumes of the high-pressure helium (hereinafter referred to as $\mathrm{He}$ ) gas bottle and two gas tanks as well as approximate locations of various sensor measuring points in the actual system are given. Modularization modeling divides the system into 2 fluid sources (FS1-2), 10 gas pipes (GP1-10), 4 gas volumes (GVol1-4), 2 gas valves (GV1-2), 1 gas pressure reducing regulator (GPRR1) and 2 check valves (CV1-2). The flow field grids of pipe-type components are generated by $100 \mathrm{~mm} / \mathrm{grid}$ and adiabatic model or radial $1 \mathrm{D}$ heat transfer model is used for pipe wall; the length along flow direction of lumped parameter components (such as gas valve, gas volume, etc.) is two standard grid units (1 unit for initial and terminal components), i.e. $200 \mathrm{~mm}$. The outlet-fluid-source boundary condition for FS1 and FS2 is constant-pressure atmospheric environment.

The PRR and the check valves in two tank pressurization pipelines are three movable components in the process of system operation. When two outlet valves of system are unopened, valve spools of the PRR and CV are fully closed under the action of forces, which divides the whole system into sections with different thermophysical states. After opening of the two valves, pressures of the pipeline networks where two tanks are located gradually decrease with He gas outflow; when the tank pressures decrease to a certain point, valve spools of the PRR and CV open under the action of differential-pressure force and then the whole system gradually enters rated working condition. At the end of the combined test, two valves close and He gas stops flowing out, so tank pressures gradually go up with pressurization of He; when tank pressures increase to a certain point, valve spools of the PRR and CV close and then the whole system is divided into sections with different thermophysical states again. The gas pipe flow is considered as 1D ideal gas flow and the quasi steady-state average friction loss model is used for pipe wall friction. GV1 and GV2 adopt the pressure ratio-based injector orifice model [38] to calculate throttling and the variable-coefficient default formula scheme [37] to calculate flow coefficient. Numerical simulation is conducted for the abovementioned dynamic process by adopting the constant physical-property controller and the classic four-stage fourth-order Runge-Kutta method.

\subsection{Experiment information}

The double-valve combined test has different working conditions by initial pressure of high-pressure He gas bottle. The measured data of an experiment of 9MPa working condition are shown in Fig. 12, where GPRR1_p1 and GPRR1_p2 are $p_{0}$ and $p_{1}$ of pressure measuring points in front of and behind GPRR1 as shown in Fig. 11, respectively; CV1_p2 and CV2_p2 are $p_{2}$ and $p_{3}$ of pressure measuring points behind CV1 and CV2, respectively; Qm1 and Qm2 are $Q_{\mathrm{m} 1}$ and $Q_{\mathrm{m} 2}$ of flow-rate measuring points behind CV1 and CV2, respectively. The data acquisition frequency stands at $1000 \mathrm{~Hz}$, that is, a data point per 1 millisecond (ms). The data are pretreated to filter the noise in order to realize data smooth processing. For the purpose of portability, all sensors are concentrated in a test box. The pressure sensors of $p_{0}$ and $p_{1}$ in the test box connect with main pipeline through flexible metal hoses with the length of about $2 \mathrm{~m}$, respectively. The type of pressure sensors is HM22-3-A1-F1-W1, and the overall accuracy is $\pm 0.25 \% \mathrm{FS}$, the response time is less than $2 \mathrm{~ms}$. The type of mass flow meters is $5860 \mathrm{E}$ thermal mass flow meter, and the accuracy is $\pm 1 \% \mathrm{FS}$, the response time is less than $3 \mathrm{~s}$ response to within $2 \%$ of full scale final value for a 0 to $100 \%$ command step.

As shown in Fig. 12, the combined test system roughly has the following working process in this 0-540s test:

1) 0-about 9.2s: the standby working condition when two outlet valves GV1 and GV2 are unopened;

2) About 9.2-20s: the startup working condition when valve spools of GV, PRR and CV open;

3) About 20-475s: the rated pressurization working condition when two tank pressures recover to and keep in 
the rated value under the effect of gas pressurization;

4) About 475-490s: the shutdown working condition when valve spools of GV, PRR and CV close;

5) About 490-540s: the halt working condition after the PRR and CV spools completely close.

\subsection{Initial, structure and control parameters settings}

The initial state before the system startup: the pipeline between high-pressure He gas bottle and GPRR1 high-pressure chamber (Pipeline network I ) is $p_{0}$; the pipelines among GPRR1 low-pressure chamber and CV1 and CV2 high-pressure chambers (Pipeline network II) are $p_{1}$; the pipelines between CV1 and CV2 low-pressure chambers and GV1 and GV2 upstream chambers (Pipeline networks III and IV) are $p_{2}$ and $p_{3}$, respectively; pressures of the $\mathrm{CV}$ opening and non-return chambers are average value of pressures of high- and low-pressure chambers; pressures of the pipelines behind downstream chambers of GV1 and GV2 (Pipeline network V) are equal to the environmental atmospheric pressure $p_{\text {atm }}$; temperature of the whole pipeline is $293.15 \mathrm{~K}$.

According to the test data, the parameter settings of the simulation case are shown in Table 2. $M_{\Delta t}$ is magnitude of time step. In GPRR1, $d_{\mathrm{v} 3}$ and $d_{\mathrm{v} 4}$ are diameters of feedback and unloading orifices of the PRR, respectively; $C_{\mathrm{d} 1}, C_{\mathrm{d} 3}$ and $C_{\mathrm{d} 4}$ are flow coefficients of throttling points of valve spool, feedback orifice and unloading orifice, respectively. In GV1 and GV2, $d_{\mathrm{vs}}$ is diameter of flow section under the condition of being equivalent to round orifice when the valve is completely opened; $C_{\mathrm{d}}$ is valve flow coefficient which changes with valve opening; $t_{\text {test }}-\tau_{\mathrm{vs}}$ is operating time sequence of the two valves in the double-valve combined test obtained by analyzing the test curves. The lock-up pressure of GPRR1 is $1.64 \mathrm{MPa}$; the cracking pressure of $\mathrm{CV} 1,2$ is $0.15 \mathrm{MPa}$.

\section{Results and discussion}

\subsection{Simulation results and analysis of system with considering the wall heat transfer}

Pipe-wall radial 1D heat transfer model is used for four types of module components including GP, GV, GPRR and $\mathrm{CV}$, and pipe-wall $\mathrm{OD}$ heat transfer model with considering environmental heat transfer for GVol module components. For components which use 1D heat transfer model, the total number of wall radial grid sequence number is 4 . The heat transfer between exterior walls of components and the external environment is considered as natural convection. The external environment temperature is $293.15 \mathrm{~K}\left(20^{\circ} \mathrm{C}\right)$, and the coefficient of convective heat transfer with normal-temperature environment is set as $4 \mathrm{~W} /\left(\mathrm{m}^{2} \mathrm{~K}\right)$. The simulation results of various components are shown in Fig. 13-Fig. 20 which are listed in the sequence of component serial number shown in Fig. 11. Data output frequency is $2000 \mathrm{~Hz}$, i.e., a data point per $0.5 \mathrm{~ms}$ at system startup and shutdown stages; $10000 \mathrm{~Hz}$, i.e., a data point per $0.1 \mathrm{~ms}$ at valve-spool opening and closing stages of GPRR and CV; $100 \mathrm{~Hz}$, i.e., a data point per 0.01 second (s) at transition stages; $10 \mathrm{~Hz}$, i.e., a data point per $0.1 \mathrm{~s}$ at initial, rated condition and final stages.

It can be seen from dynamic curves of GPRR1 (Fig. 14), CV1 (Fig. 15) and CV2 (Fig. 16), GVol3 and GVol4 (Fig. 18), GV1 and GV2 (Fig. 20): as the system outlets are constant-pressure atmospheric environment and there is not a real-time control mechanism, the sole driver for dynamic change of system state is the pressure changes of two pipelines behind two check valves represented by two gas tanks with outflow and pressurization supplement of He gas, which are triggered by the opening and closing of two outlet valves. Below is analysis by stage.

Stage 1: The standby stage

As two outlet valves GV1 and GV2 keep unopened before 9.2s, all state parameters of system including main pipeline, GVol3 tank pressurization pipeline (hereinafter referred to as the left pipeline) and GVol4 tank pressurization pipeline (hereinafter referred to as the right pipeline) stay at standby flat state.

Stage 2: The stage of tank blowdown mode

Exit pressures begin to decrease in the right pipeline after 9.32s (Fig. 16c) and in the left pipeline after 9.40s (Fig. $15 \mathrm{c})$, which indicates that the system begins to start up from standby state. As the tank pressure has not decreased to the point which is enough to make small valve spool of CV2 or CV1 open during a period of time after startup, only He gases in the pipelines between CV2/CV1 low-pressure chambers and GV2/GV1 upstream chambers flow to atmospheric environment. Accordingly, only pressures in the two pipelines gradually go down while state parameters of the pipelines in front of CV2 and CV1 non-return chambers continue to stand by. At this time, GVol4 and GVol3 are equivalently in tank blowdown mode (i.e. pressure-dropping working condition).

Stage 3: The critical opening stage of CV spools

With two-pipeline tank pressures decreasing, the pressure differences between upstream and downstream of the CV small valve spools successively reach critical opening pressure difference (COPD, i.e. the minimum differential pressure for opening) at 11.10 s for CV2 (Fig. 16c, e and g) and 11.27s for CV1 (Fig. 15c, e and g). However, 
$\cdot 14 \cdot$

because big spools are still closed, the CV small spools are in the critical opening state of slightly alternately opening and closing in fully closed position, that is: when the differential pressure in front of and behind the small spool is higher than the COPD, the small spool slightly opens and the retained gas in non-return and opening chambers flows out; then the small-spool pressure difference decreases, and the small spool shuts down again under the action of spring force. As the initial pressure behind the CV of the right pipeline is lower than that of the left pipeline (that is, $p_{3}<p_{2}$ in Table 2), in the case when structures and spring preload forces of two check valves are the same completely, the pressure difference of CV2 big spool firstly reaches the COPD at 12.98s (Fig. 14c and e, Fig. 15c, Fig. 16c, e and g). Then the pressure of Pipeline network II (i.e. the PRR-CV pipelines) begins to decrease. Meanwhile, the pressure difference of CV1 big spool keeps lower than the COPD because the high-pressure-chamber pressure of CV1 is equal to that of CV2 and the low-pressure-chamber pressure of CV1 is higher than that of CV2. Therefore, in the case when the PRR valve spool has not opened, the gas in the PRR-CV pipelines only flows to the right-pipeline tank GVol4 and finally out of the system through the critical opening and closing of CV2 big and small spools, while CV1 big spool keeps unopened at this stage (Fig. 15e and g).

\section{Stage 4: The opening stage of the PRR and CV spools}

Until 15.39s (Fig. 14c-f), with the decrease of pressures of PRR low-pressure and feedback chambers, the PRR valve spool enters opening process as the pneumatic closing force acting on the spool is smaller than the spring opening force. During initial opening stage around 15.39-15.41s (Fig. 14d, Fig. 16d and f), as the feedback-chamber pressure decreases very slowly and there are tiny oscillations approximately in form of periodic sine wave in the gas flows of PRR and CV low-pressure chambers, openings of GPRR1 and CV2 valve spools only fluctuate within a very small range until $15.41 \mathrm{~s}$ when the opening process of CV2 big spool is triggered (Fig. 16d).

In the opening process of CV2 big spool, CV2 high-pressure-chamber pressure $p(1)$ rapidly goes down, opening-chamber pressure $p(4)$ rapidly goes up, and non-return-chamber pressure $p(3)$ also goes up but at a speed obviously lower than $p(4)$ curve. Therefore, the resultant force on CV2 big spool is always greater than zero in the early opening stage, and big spool gradually opens at an increasing speed. Meanwhile, increase of $p(3)$ also causes the opening of CV2 small spool. With dynamic changes in values of $p(1), p(4), p(3)$ and $p(2)$, the resultant force on CV2 big spool is less than zero in the later stage. However, because of having reached a certain opening velocity, the big spool stays in a state of positive velocity until complete opening $\left(h_{1}=h_{\max 1}=1 \mathrm{~mm}\right)$ even though the closing force makes it have sharp slowdown after 15.4163s (Fig. 16f). Then, $p(4)$ and $p(1)$ tend to concord and their values go up after down at 15.4189s (Fig. 16d) with supplement of gas flowing from the PRR upstream; $p(3)$ and $p(2)$ tend to concord; the big spool stabilizes in the fully open position.

The opening process of CV2 small spool is similar to that of big spool. Differently, the alternating phenomenon of rise and decrease of the small-spool opening velocity is more obvious with dynamic change of $p(3)$ and $p(2)$, and even there are several stages when the movement velocity is negative and the opening decreases (Fig. 16f). Although the resultant force on the small spool is negative at late opening stage due to decrease of $p(3)$ and rise of $p(2)$, big and small valve spools contact and adhere to each other after about 15.4176s and the total resultant force on them is positive. Therefore, two valve spools bond together to continue to open in later stage until $15.4189 \mathrm{~s}$ when $h_{1}=h_{\max 1}, h_{2}=h_{\max 1}-h_{\max 3}=0.5 \mathrm{~mm}$ (Note: the small spool is not fully open when $h_{2}=h_{\max 2}=1 \mathrm{~mm}$.). Then the big spool sticks against the rear valve seat and stops moving, but the small spool continues opening movement due to inertia until $15.4194 \mathrm{~s}$ when it reaches the maximum opening of $0.571 \mathrm{~mm}$. During the process, the small spool slows down gradually due to action of closing force. At $15.4194 \mathrm{~s}$, its inertia runs out and velocity reverses. Then, the openings and velocities of small and big spools slightly fluctuate around 15.4199-15.4205s with fluctuations of pressures in various chambers. Until $15.4205 \mathrm{~s}$, the small spool backs to adhesion with big spool and is stabilized.

Because the pressure behind $\mathrm{CV}$ of the left pipeline is always a little higher than that of the right pipeline from 0s to $15.41 \mathrm{~s}$ when CV2 big spool begins to open, and the pressures in the PRR-CV pipelines represented by CV2 high-pressure chamber pressure $p(1)$ keep decreasing from $15.41 \mathrm{~s}$ to 15.4189 s (Fig. 14d, Fig. 15d, Fig. 16d), the CV1 big spool does not open at this stage due to its less differential pressure than the COPD. At 15.4189s, $p(1)$ begins to rise due to the supplement of gas from PRR upstream and reaches its peak value around 15.438s. This means the pressure difference of CV1 reaches the COPD, so CV1 big spool enters its opening process (Fig. 15d and f) which is similar to that of CV2 big spool.

The opening process of CV1 small spool is different from that of CV2 small spool but similar to that of CV1 big spool. With dynamic changes of $p(3)$ and $p(2)$, the opening velocity of CV1 small spool mostly keeps increasing except a short period of decrease during 15.4423-15.4432s and is always positive. After 15.4433s, the big and small spools contact and bond together to continue to open. Until 15.4438s when big spool reaches the maximum opening, the big spool sticks against the rear valve seat and stops moving, while the small spool continues opening movement due to inertia until $15.4446 \mathrm{~s}$ when it reaches the maximum opening of $0.648 \mathrm{~mm}$. The dynamic process during 15.4446-15.4462s is similar to that of CV2 small spool.

So far, big and small spools of CV2 and CV1 have completed their opening and enter the steady state in which 
big spool of each CV is fully opened and two valve spools adhere to each other. That means the big and small spools are pressed on the rear seat of big spool by the resultant force of various chamber pressures and spring forces. It should be noted that: as the CV modeling uses the assumption that collisions among big spool, small spool, and valve seats are completely inelastic collision, changes in openings of various spools obtained by simulation are simplified somewhat; the real physical process should be more complicated due to the nature of elastic collision.

As indicated by Fig. 14d, the PRR unloading-chamber pressure $p(4)$ curve basically coincides with the high-pressure-chamber pressure $p(1)$ curve; since very small feedback orifice causes obvious lag phenomenon of feedback-chamber pressure $p(3)$ behind low-pressure-chamber pressure $p(2)$, the fluctuation of $p(3)$ curve is not as obvious as the $p(2)$ curve although $p(3)$ is declining on the whole. Therefore, the main factors determining the segmentation of PRR valve-spool opening process are fluctuations of $p(1)$ and $p(2)$. Due to successive opening of CV2 and CV1 spools and approximate sine-wave oscillation of pressures of the PRR high- and low-pressure chambers, the opening process of GPRR1 valve spool is divided into several segments (Fig. 14d and f) as follows:

1) around 15.410-15.420s, the opening of CV2 big and small spools causes decline of the PRR-CV pipelines pressures represented by $p(2)$, so the PRR spool opens at an increasing speed as a whole;

2) around $15.420-15.434 \mathrm{~s}$, as $p(1)$ and $p(2)$ enter the rising stage, the PRR spool switches to the state of opening at a decreasing speed as a whole. During the process, the spool opening velocity has fluctuation which is mainly caused by the fluctuation of $p(2)$;

$3)$ at $15.4342 \mathrm{~s}, p(2)$ approaches the peak value of this wave segment, and $p(1)$ and $p(4)$ enter the rising stage (Both the force of $p(2)$ and the resultant force of $p(1)$ and $p(4)$ are in closing direction acting on PRR spool.), so the PRR spool velocity $h_{\mathrm{d}}$ goes negative from positive. This means the spool gets closing from opening, and then the spool opening $h$ decreases during 15.4342-15.4404s;

4) around 15.438-15.446s, the opening of CV1 big and small spools causes decline of the PRR-CV pipelines pressures again, so the PRR gains positive acceleration once more, then the spool closing inertia gradually runs out and $h_{\mathrm{d}}$ becomes positive from negative at $15.4404 \mathrm{~s}$ which means the spool returns to the state of accelerating opening; after that, with $p(1), p(2)$ and $p(4)$ continuing oscillation of approximate sine-wave periodic damping, the PRR spool gradually opens to its rated opening;

5 ) at about $15.55 \mathrm{~s}$, the oscillation of $p(2)$ curve decays to unapparent state. Meanwhile, the PRR spool is stabilized at the opening point of $0.0118 \mathrm{~mm}$ and its oscillation state switches to unapparent high-frequency small-amplitude oscillation as shown in Fig. 14c and d (The amplitude and frequency of the high-frequency oscillation have relationship with the PRR structure and size. A PRR with good structure and size has weak oscillation or even no oscillation while bad structure and size can cause obvious oscillation. [39]). So far, the opening process of GPRR1 spool is completed.

Stage 5: The recovery stage of two tank pressures

After opening of the PRR and CV valve spools, the PRR begins to output pressurization He gas of rated output pressure around 1.64MPa. The right and left pipelines switch to pressure regulated mode (i.e., pressurization working condition) from tank blowdown mode around $15.41 \mathrm{~s}$ and $15.44 \mathrm{~s}$, respectively. Pressures and temperatures of two gas tanks GVol4 and GVol3 begin to recover gradually with supply of two-pipeline pressurization gases, and the recovery velocities get slower with increase in tank pressures and temperatures and become unapparent after about 60 s as shown in Fig. 18. Table 3 shows the recovery history of pressures and temperatures of two tanks.

It is noteworthy that: in the case of PRR rated output pressure being approximately constant, the low-to-high tank pressure causes high-to-low pressure difference between upstream and downstream of CV2 or CV1. Meanwhile, the $\mathrm{CV}$ non-return orifice is in the subcritical throttling condition where its pressure distribution does not reached critical throttling condition (i.e. choked condition). Therefore, the He flow rate is affected by both non-return orifice upstream and downstream state parameters, which finally makes the mass flow rates of two-pipeline pressurization gases go low from high. Mass-flow-rate curves in many figures such as Fig. 14a, Fig. 15a, Fig. 16a, Fig. 17a and Fig. 18 all obviously reveal this phenomenon. As shown in Fig. 17a, in contrast to the variation trend of tank pressure recovery curves, the pressurization-gas mass-flow-rate curves of the left pipeline gradually go down after experiencing the flux peak and brief damped oscillation around 15.44s. In addition, the flow-rate decrease speeds get slower with gradually slower increase speeds of tank pressures and become unapparent after about 60s. Table 4 shows the variation history of GP4 outlet flow rate.

Stage 6: The working stage of stable tank pressures

After the tank pressures recover to stable condition, the system enters pressurization working stage when the tank pressures are relatively stable around $60-475 \mathrm{~s}$. The whole gas system is basically divided into the following five working states by PRR, check valves, gas tanks and outlet valves:

Pipeline network I : Pressures, densities and temperatures of grids in the pipeline between GVol1 and GPRR1 high-pressure chamber gradually go down with He consumption (Fig. 13a, Fig. 14a and b);

Pipeline network II : Flow-field and wall temperatures of grids in the PRR-CV pipelines gradually decline (Fig. 
13-Fig. 16b); flow-field densities gradually go up (Fig. 13b); pressures and mass flow rates slightly increase (Fig. 13b, Fig. 14-Fig. 16a). The slight increase phenomenon of He mass flow rates around 55-474s attributes to the gradual rise in densities caused by gradual decrease of He temperatures as well as the gradual rise in pressure obtained by ideal gas calculation formulas;

Pipeline network III: Variation trends of flow-field pressures, densities, temperatures, mass flow rates and pipe-wall temperatures of grids in the pipelines between CV1 or CV2 low-pressure chamber and GP4 or GP8 outlet are the same as those in Pipeline network II except that the decline speeds of temperatures are slower than those of Pipeline network II (Fig. 15b, Fig. 16b, Fig. 17);

Pipeline network IV: Variation trend of pressures in the pipelines between GVol3, 4 and GV1, 2 upstream chamber is similar to that in Pipeline network II, i.e. slight increase (Fig. 18, Fig. 20). However, the flow-field temperature curves first climb and then decline with about 135-160s as dividing line (The upstream grids have the transition earlier than the downstream grids.); variation characteristics of wall temperatures are similar to those of the flow-field temperatures (Fig. 19a). On the contrary, the density curves first decline and then go up with about 125-140s as dividing line;

Pipeline network V: Pressures in the pipelines behind GV1, 2 downstream chamber are close to the atmospheric environment pressure and thus are basically flat during 60-475s (Fig. 20); Variation trend of flow-field temperature, densities and wall temperatures (Fig. 19b) are similar to those in Pipeline network IV.

It is interesting to note that: among the abovementioned five sections of pipeline networks, temperature drop in Pipeline network I is most apparent, and followed by Pipeline network II which is followed by Pipeline network III. In Pipeline networks IV and V, the temperature has ended its pure downward trend and shows a down-after-up trend, and the overall variation is relatively small.

Stage 7: The tank-pressure rising stage after the closure of outlet valves

The working stage of stable tank pressures lasts until about $475 \mathrm{~s}$. With the closing of outlet valves GV2 at 474.92-475.13s and GV1 at 474.94-475.20s, He gases stop flowing out and tank pressures enter a rising stage, which triggers a series of changes as follows: tank pressures go up (Fig. 18) $\rightarrow$ CV low-pressure chamber pressures $p(2)$ rise (Fig. 15i, Fig. 16i) $\rightarrow \mathrm{CV}$ small-spool flow rates $Q_{\mathrm{m}}(3)$ decline (Fig. 15h, Fig. 16h) $\rightarrow$ CV non-return chamber pressures $p(3)$ rise $\rightarrow \mathrm{CV}$ non-return orifice flow rates $Q_{\mathrm{m}}(4)$ decline $\rightarrow \mathrm{CV}$ opening chamber pressures $p(4)$ rise $\rightarrow \mathrm{CV}$ big-spool flow rates $Q_{\mathrm{m}}(1)$ decline $\rightarrow \mathrm{CV}$ high-pressure chamber pressures $p(1)$ rise $\rightarrow$ PRR low-pressure chamber and feedback chamber pressures rise (Fig. 14g) $\rightarrow$ PRR valve-spool opening $h$ decreases $\rightarrow$ PRR valve-spool flow rate $Q_{\mathrm{m}}(1)$ drops (Fig. 14i). Although the method of causality analysis is employed in describing the above changes, the turning points of the abovementioned state-parameter curves are actually almost synchronous, and there is no obvious time sequence which can be seen from these figures.

It is interesting to note that: during the series of continuous changes around $475-485 \mathrm{~s}$, the pressures of various chambers are on the rise and the mass flow rates of big and small spools are on the decline for two check valves, but the openings of big and small spools always keep in the state of $h_{1}=h_{\max 1}$ and $h_{2}=h_{\max 1}-h_{\max 3}$, respectively and do not have any change, which is obviously different from the situation that the PRR valve-spool opening decreases gradually. In fact, this is a result of difference in working principle between PRR and CV: for the PRR, the valve spool works in a dynamic-balance state point within $0<h<h_{\max }$, i.e., in a dynamic force equilibrium of acting forces of various chamber fluids and elastic components, so any pressure disturbance will cause the disturbance of its valve-spool opening (The PRR used in the system has incomplete pressure unloading for high- or low-pressure chamber pressure, so the pressure of each chamber has an impact on the valve spool.). What is more, what continues to rise around 475-485s is PRR feedback-chamber pressure which plays a leading role in the state of spool opening due to its directly acting on the large-area diaphragm. These lead to the gradual decrease of the PRR opening; for the $\mathrm{CV}$, the valve spools work in a stationary state where big spool is fully opened and two valve spools adhere to each other, i.e., big spool is pressed on its rear seat by positive resultant force of various chamber pressures and spring forces and small spool is pressed on big spool by opposite resultant force of spring force and various chamber pressures. If the action of big-spool rear seat was removed, big and small spools would not be in a dynamic force equilibrium of various chamber pressures and spring forces. Therefore, even if the downstream pressure is rising, the openings of big and small spools will not have any change so long as the downstream pressure has not reached the point which can make two valve spools close reversely.

The second characteristic worthy of attention is that: initial pressures of CV opening and non-return chambers are set to average value of pressures of high- and low-pressure chambers, i.e. $p(1)>p(4)=p(3)=[p(1)+p(2)] / 2>p(2)$, and the state of $p(4)=p(3)$ does not change at the critical opening stage; there is $p(1) \approx p(4)>p(3) \approx p(2)$ at early stage of the big spool closing. Furthermore, the areas of $p(4)$ and $p(3)$ acting on the CV spools are significantly larger than those of $p(1)$ and $p(2)$. Therefore, judging purely from the pressure distribution of various chamber gases acting on the valve spools, the $\mathrm{CV}$ has the characteristic of requiring large differential pressure distribution for 
opening and small differential pressure distribution for closing, and the fact that values of the spring closing forces before closing are slightly greater than the values before opening will slightly enhance the characteristic. Table 5 shows the pressure distribution of two check valves before opening (Fig. 16d, Fig. 15d) and before closing (Fig. 16j, Fig. 15j). In this sense of differential pressure distribution, 'hard to open but easy to close' or 'easy to close but hard to open' can be used to describe this characteristic.

The third characteristic worthy of attention is that: around 475-485s, He gases stop flowing out of two outlet valves, but the PRR and CV valve spools have not reached the closing condition of differential pressure and the pressurization gases are still flowing into two-pipeline gas tanks GVol4, GVol3 and gas pipes GP9, GP5. Therefore, pressures and temperatures of these four components (Fig. 18 and Fig. 19a) go up, which coincides with the phenomena that temperature of a gas bottle increases when inflated and decreases when deflated (the inflated and deflated effects). Meanwhile, wall temperatures of GVol4,3 are also on the rise due to the heating effect of the environment at early stage and the dual heating effects of the fluid and the environment at late stage.

\section{Stage 8: The closing stage of the PRR and CV spools}

As the tank pressures are continually increasing and the pressure behind CV2 is slightly higher than that behind $\mathrm{CV} 1$, so the right-pipeline CV2 first enters the closing stage of valve spools (Fig. 16j and 1): during 485.850-485.9357s, big and small spools bond together to close at an increasing speed; at $485.9357 \mathrm{~s}$, with the rise of $p(1)$ and $p(4)$, the resultant force of spools switches to positive from negative and thus the spools switch to the state of closing at a decreasing speed; at 485.9358s, CV2 small spool hits its front seat and closes fully, and big spool separates from small spool and continues closing at a decreasing speed; with the dynamic changes of various chamber pressures, both the two spools have a period of transient reversal from closing to slightly opening around $485.9362 \mathrm{~s}$ and they adhere to each other again at 485.9363s; then big spool switches to the state of closing at an increasing speed due to the rise of $p(3)$ and separates from small spool and then continues closing; around some time points such as $485.937 \mathrm{~s}, 485.941 \mathrm{~s}, 485.946 \mathrm{~s}$ and $485.951 \mathrm{~s}$, big spool shows the closing state of acceleration and deceleration alternating with the fluctuations of $p(1), p(4)$ and $p(3)$; until $485.9514 \mathrm{~s}$, big spool closes completely. So far, CV2 spools have completed their closing process.

After the CV2 spools begin to close, as the pressure behind CV1 is only a little lower than that behind CV2 (For example, the values of pressures $p(2)$ of CV1 and CV2 are $1.6237 \mathrm{MPa}$ and $1.6238 \mathrm{Mpa}$, respectively at $485.850 \mathrm{~s}$.), Fig. $15 \mathrm{j}$ and 1 show that the left-pipeline CV1 also enters a small closing stage during 485.886-485.916s. However, the large-scale closure of CV2 spools results in the gradual increase of the PRR-CV pipelines pressures, and the pressures reach a relatively high peak though an obvious rising sine curve during 485.924-485.942s. Therefore, CV1 big and small spools open again and return to the previous opening state of $h_{1}=h_{\max 1}$ and $h_{2}=h_{\max 1}-h_{\max 3}$. It can be said that the first closing of CV2 spools delays the formal closing time of CV1. Until 485.958s when the PRR-CV pipelines pressures return to the normal value through an obvious sine decline curve after CV2 closes completely, CV1 big and small spools enter a formal closing stage. Its closing process is similar to that of CV2 spools, while the difference is that the CV1 spools show the closing state of acceleration and deceleration alternating at early and middle stages during 485.958-486.0215s. At 486.0376s, CV1 big spool closes completely.

So far, both CV2 and CV1 have completed the closing processes and big and small spools of each CV are pressed on their own front seats by the resultant force of spring force and various chamber pressures. Due to successive closing of CV2 and CV1 and approximate sine-wave oscillation of pressures of the PRR high- and low-pressure chambers, the closing process of GPRR1 valve spool is divided into several segments (Fig. 14h and j) as follows:

1) around 485.92-485.942s, the closing of CV2 spools causes rise of the PRR-CV pipelines pressures, so the PRR spool closes at an increasing speed as a whole. During the process, the spool closing velocity has fluctuation which is mainly caused by the fluctuation of $p(2)$;

2) around 485.946-485.960s, as $p(1)$ and $p(2)$ enter the decline stage, the PRR spool switches to the state of closing at a decreasing speed as a whole; at $485.957 \mathrm{~s}$, as $p(2)$ approaches the valley value of this wave segment, and $p(1)$ is also on the decline, the PRR spool velocity $h_{\mathrm{d}}$ goes positive from negative. This means the spool gets opening from closing, and then the spool opening $h$ increases during 485.957-485.964s;

3) around 485.960-485.966s, the formal closing of CV1 spools results in rise of the PRR-CV pipelines pressures again, so the PRR gains negative acceleration once more, then its opening inertia gradually runs out and $h_{\mathrm{d}}$ becomes negative from positive at $485.965 \mathrm{~s}$ which means it returns to the state of accelerating closing; after that, with $p(1)$ and $p(2)$ continuing oscillations in approximate sine-wave periodic form, the PRR spool is in the state of alternate acceleration and deceleration and alternate closing and opening, which causes that $h$ has little change as a whole;

4) until about $486.02 \mathrm{~s}$, with the complete closure of CV1 small spool, the PRR spool begins to close at an increasing speed once again; then $h$ decreases rapidly and the PRR closes fully at 486.0330s. After CV1 big spool also closes fully at $486.0376 \mathrm{~s}$, the PRR $p(2)$ goes down and $p(3)$ goes up, and the two pressure curves gradually converge and overlap with each other at $486.160 \mathrm{~s}(1.6564 \mathrm{MPa})$. So far, the system enters the halt stage after the PRR and CV spools completely close. 
Many state parameters of various grids do not stay at a flat unchanged state in the halt stage as they do in the initial standby stage. The main factor causing changes is the effect of heat transfer between the fluid in the pipeline network and external constant-temperature environment through wall. Table 6 shows the variation and distribution of system pressures and temperatures (Fig. 13a, Fig. 17, Fig. 18a and Fig. 19). The whole system is basically divided into the following four working states by the valve spools of PRR, check valves and outlet valves:

Pipeline network I : Fluid-field and wall temperatures (except GVoll wall temperature) go up slowly due to the heating effect of external environment and the pressures also increase accordingly;

Pipeline network II : Similar to Pipeline network I, flow-field and wall temperatures in the PRR-CV pipelines go up slowly due to heating effect of external environment and the pressures also increase accordingly;

Pipeline network III and IV: The grids in these pipeline networks have two working states: flow-field and wall temperatures of GP4, GP5, GP8 and GP9 go up slowly due to the heating effect of external environment; since flow-field temperatures of two tanks rise to values higher than the environment temperature $(293.15 \mathrm{~K})$ due to the gas-bottle inflated effect in the closing stage of PRR and CV spools, He gas temperatures in GVol3, 4 gradually drop with releasing heat toward the environment through their walls in the halt stage. As the volumes of two tanks are relatively large in their own pipelines, Pipeline network III and IV are releasing heat toward the environment on the whole and the total energy of gases in it is on the decline. Therefore, in contrast to the change trend of pressures in Pipeline network I and II, pressures of various components decline synchronously;

Pipeline network V: Fluid-field and wall temperatures go up slowly due to the heating effect of external environment; the pressures coincide with the atmospheric environment pressure and thus are basically flat.

\subsection{Comparison of simulation results under two wall heat transfer conditions with experimental results}

Fig. 21-Fig. 23 show comparison of system simulation results with experiment-measured results at various measuring points under two simulation conditions of adiabatic wall and considering wall heat transfer. In these figures, the curves marked with symbols like triangle, square, etc. are experimental data while the curves without marks are simulation results of component grids corresponding to sensor measuring points.

As described in section 3.2, test curves divide 0-540s working process of the double-valve combined test system into five working conditions: standby, startup, rated pressurization, shutdown and halt. It can be seen from above analysis on simulation results that the five working conditions can be subdivided into the following nine stages:

1) 0-about 9.2s: the standby stage when valve spools of the outlet valves, PRR and CV are unopened;

2) About 9.3-11.1s: the stage of tank blowdown mode (i.e., pressure-dropping working condition) when two-pipeline outlet valves are open but the PRR and CV spools are unopened;

3) About 11.1-15.4s: the critical opening stage when the CV spools slightly open and close alternately in their own fully closed positions;

4) About 15.4-15.5s: the opening stage of the PRR and CV spools (CV2 spools firstly open and then CV1 spools open, which causes GPRR1 spool to mainly have two opening stages.);

5) About 15.5-60s: the recovery stage when two tank pressures recover to rated value under the effect of gas pressurization;

6) About $60-475 \mathrm{~s}$ : the working stage of stable tank pressures;

7) About 475-485.8s: the tank-pressure rising stage after the closure of outlet valves;

8) About 485.8-486.1s: the closing stage of the PRR and CV spools (CV2 spools firstly close and then CV1 spools close, which causes GPRR1 spool to mainly have two closing stages.);

9) About 486.2-540s: the halt stage after the PRR and CV spools completely close.

In Fig. 21-Fig. 23, test curves and simulation curves of pressures of the PRR high- and low-pressure chambers, downstream pressures and mass flow rates of the check valves all basically show the above nine stages. The simulation is well consistent with the test as a whole. However, the test system have no measuring points for CV state parameters, thus the test data are unable to clarify the critical opening stage of CV spools (Stage 3). Meanwhile, the test cannot accurately show the opening and closing details of the PRR and CV spools. The heat-transfer-wall simulation results are closer to the test curves than adiabatic-wall results and are more able to reveal some change characteristics of the system. Below is detailed analysis according to every figure.

As shown by Fig. 21a-f, for GPRR1 high-pressure chamber pressure $p(1)$, the adiabatic-wall simulation curve goes down a lot faster than the test curve in the working stage of stable tank pressures (Stage 6) and gets flat in the halt stage (Stage 9), while the test curve slowly goes up after a short flat in Stage 9; the heat-transfer-wall simulation curve goes down at a speed which is close to the test curve in Stage 6, and also obtains the phenomenon of flat first and then slowly rising in Stage 9. For the PRR low-pressure chamber pressure $p(2)$, the adiabatic-wall simulation curve goes up obviously faster than the test curve in Stage 6, while the heat-transfer-wall simulation curve has 
slower rising trend and thus is closer to the test curve.

In addition, Fig. 21c and d show that both simulation curves and test curve give two time points for the right-pipeline CV2 big spool entering critical opening stage and the PRR spool starting to open, and the two time points in Fig. 21d are closer to the test data than those in Fig. 21c. However, on the one hand, Fig. 21c and Fig. 22c show that the adiabatic-wall simulation curve of $p(1)$ oscillates violently during 13.6-15.4s, which causes too early opening of GPRR1 and CV2 spools. As a result, the critical opening stage of CV2 spools is obviously shortened; on the other hand, around 15.42s when CV2 completely opens, simulation curves show that a large pressure drop of PRR $p(2)$ is caused by very small feedback orifice and obvious lag phenomenon of feedback-chamber gas state behind low-pressure chamber gas state, while test curve does not show this phenomenon. The pressure drop of PRR $p(2)$ at the opening moment can always be observed during the single-component test of PRR. It does not appear in the combined test curve probably due to the reasons as follows: 1) the "drop" oscillation signal of tens of milliseconds are filtered out by the filter; 2) this signal is damped out by the $2 \mathrm{~m}$-long flexible metal hose between the pressure sensor in the test box and the main pipeline as shown in Fig. 11.

Fig. 21e and f show that both simulation and test curves give two time points for GV1,2 closing and PRR spool starting to close, but simulation curves of PRR $p(2)$ do not go up as violently as test curve which is probably due to the reasons as follows: 1) as shown in Fig. 6, valve spool stem, spool steel ball and auxiliary spring upper seat are actually three independent movement parts and are pressed to be one moving system by three springs. Since the gradual decreasing of PRR spool opening $h$ during $475-485 \mathrm{~s}$ (Fig. 14g) is induced by gradual increasing of PRR $p(2)$ and $p(3)$, valve spool stem is possible to close independently from the rest of the moving system. Thus the closing force of auxiliary spring is removed form the stem moving subsystem, and the actual values of $p(2)$ and $p(3)$ enlarge; 2) as PRR opening $h$ is approximatively inversely proportional to the flow coefficient $C_{\mathrm{d} 1}$ when high-pressure chamber pressure, temperature and rated flow are definitive, the actual value of $h$ will be greater than the simulation value if the actual value of $C_{\mathrm{d} 1}$ is less than conventional 0.9 show in Table 2. Thus the actual compression lengths of big and small springs will enlarge in the process of $h$ gradually decreasing from rating value. This means the actual increment of spring force will enlarge during 475-485s, so the actual values of $p(2)$ and $p(3)$ enlarge.

As shown in Fig. 22a-f, for CV1, 2 downstream pressures, both test curves and heat-transfer-wall simulation curves have the phenomenon of pressure gradually declining in the halt stage (Stage 9) caused by the temperature declining which results from hotter gases in the tanks releasing heat toward the environment through their walls, while the adiabatic-wall simulation curves are flat. However, the heat-transfer-wall simulation curves go up more obvious in Stage 6 than the test curves and the adiabatic-wall simulation curves. In addition, as the time points for two-pipeline outlet valves GV1 and GV2 opening and closing in the simulation case are set in accordance with the test curves, the simulation curves agree with the test curves at these points. As shown by Fig. $22 \mathrm{c}$ and d, the time points at which two simulation curves get oscillating dropping from steadily dropping disclose the time points for CV2 and CV1 small spools to enter their own critical opening states; the time point at which the oscillation amplitude heightens discloses the time point for CV2 big spool to enter the critical opening state. Although two test curves (Fig. 12b) also have fluctuations in pressure values at these time points, they can not obviously reveal the phenomena due to having no apparent oscillation characteristics; both simulation curves and test curves give the time point for the PRR spool opening. As shown by Fig. 22e and f, both simulation curves and test curves give the time point for the PRR spool closing, but the pressure simulation curves is higher than the test curves.

As shown in Fig. 23a-f, for CV1, 2 downstream gas mass flow rates, the adiabatic-wall simulation curves go up a lot faster in Stage 6 than the test curves, while the heat-transfer-wall simulation curves go up at a speed which is close to the test curves. However, there is a difference between the simulation and the test in system startup stage and shutdown stage, respectively: in the tank-pressure recovery stage (Stage 5) around 15.5-60s, two flow-rate test curves show gradual rise (Each curve has a peak ripple at a certain time point.), while the simulation curves get stable in a way of damped oscillation after a startup peak and then slowly go down; in the halt stage around 486.2-510s, the simulation curves become zero flow rates after the PRR and CV spools completely close while each test curve has a low mass-flow-rate decline stage. For the difference between simulation and test around 15.5-60s, the analysis on Stage 5 in section 4.1 has given the explanation: in the case of the PRR rated output pressure value being approximately constant, gradual rise in each tank pressure causes gradual decline in pressure difference between upstream and downstream of each CV; meanwhile, the CV non-return orifice is in the subcritical throttling condition; therefore, the mass flow rates of two-pipeline pressurization gases gradually decline. For the difference between simulation and test around 486.2-510s, it can be seen from the analysis that: the PRR and two-pipeline CV spools have closed completely after about 486.2s (The pressure curves in Fig. 21e and f, Fig. 22e and f can clearly show the completely closing characteristic.); although there is still weak flow in the pipeline network caused by the temperature difference of $\mathrm{He}$ gases, the He mass flow rate resulting form the weak flow is very slight; therefore, the He flow rate in the pipeline network should approximate zero. From the analysis on the above two differences, the simulation curves in the two stages are more reasonable while the flow-rate test curves are very probably distorted due to problems in measuring equipment and principle of two thermal mass flow meters, which has been recognized 
by engineering test personnel. In addition, Fig. $23 \mathrm{c}$ and d show that the time points when two flow-rate simulation curves get pulsating from zero flat reveal the time points when CV2 and CV1 small spools enter their own critical opening states, and the time point when the oscillation amplitude increases gives the time point when the right-pipeline CV2 big spool enters the critical opening state. However, two test curves (Fig. 12b), without obvious oscillation characteristics, are unable to disclose these characteristics; both simulation curves and test curves give the time point when GPRR1 spool opens. Fig. 23e and f show that both simulation curves and test curves give the time point when the PRR spool closes.

\section{Conclusions}

1) The developed system-level basic equations for two basic phenomena, flow and heat transfer, and the derived model system form a unified flow/heat transfer simulation system, from which the flow-field and wall temperature-field models of common components (pipe, chamber and branch, valve, etc.) and various complicated components (PRR, CV, etc.) in pipeline system can be further derived in combination with throttling model of valve spool and orifice. The system has a certain theoretical significance in its logic self-consistency and generality and a broad engineering application prospect in pipeline system simulation field.

2) The working process of the double-valve combined test system can be roughly divided into five working conditions which can be subdivided into nine stages. Simulation analysis revealed comprehensively and clearly all stages and every dynamic process, and most simulation results agree well with the experiment. Meanwhile, available test data can not clearly clarify such stages as the critical opening stage of CV spools, such details as the opening and closing process of the PRR and CV spools, such effects as temperature drop and rise, and such dynamic characteristics as the CV characteristic of 'hard to open but easy to close' and the CV downstream gas mass-flow-rate characteristics around 15.5-60s and around 486.2-510s. In this sense, a virtual test platform with certain reliability is provided and is supposed to play a useful role in actual design and experiment.

3) As the flow fields of various components in gas pipeline network have obvious effects of temperature drop and temperature rise with helium gas flowing, the simulation results when considering component wall heat transfer are closer to the test data and more reasonable than the simulation results under the adiabatic-wall condition.

4) The opening and closing process of the PRR spool and the CV big and small spools is a kind of complicated physical process of coupling interaction of many high- and low-frequency phenomena, which needs relatively precise physical model and high output frequency to accurately and clearly illustrate. However, high output frequency means the simulation data files need large hard-disk space and makes data post-processing more difficult, thus the output frequency need to be balanced with comprehensive consideration. The method of time-segmented setting different data output frequencies can not only reduce consumption of hard disk but also accurately disclose the opening and closing process of the PRR and CV spools.

\section{Acknowledgments}

This work was financially supported by the National Natural Science Foundation of China (No. 11101023) and the China Scholarship Council (No. 201203070237). The authors would like to thank Dr. Peng Xu from BUAA for his support in software interface and Professor Haixing Wang from BUAA and the anonymous referees for their revision advice.

\section{References}

[1] H. Karimi, A. Nassirharand, A. Zanj, Integration of modeling and simulation of warm pressurization and feed systems of liquid propulsion systems, Acta Astronautica 69(5-6) (2011) 258-265.

[2] N. Bhuwakietkumjohn, T. Parametthanuwat, Application of silver nanoparticles contained in ethanol as a working fluid in an oscillating heat pipe with a check valve (CLOHP/CV): a thermodynamic behaviour study, Heat Mass Transfer 51(9) (2015) 1219-1228.

[3] A. Hedayat, K.C. Knight, R.H. Champion Jr., Transient analysis of pressurization and pneumatic subsystems of the X-34 main propulsion system, AIAA 2000-3720, 2000.

[4] S. Choi, T.Y. Kim, H.K. Kim, et al, Combustion characteristics of gaseous $\mathrm{CH}_{4} / \mathrm{O}_{2}$ coaxial jets in a model combustor, AIAA 2015-3868, 2015.

[5] H. Kang, E. Lee, Y. Moon, et al, Development status of $500 \mathrm{~N}$ scale green hypergolic bipropellant thruster using hydrogen peroxide as an oxidizer, AIAA 2015-4062, 2015.

[6] R.P. Prickett, G.P. Purohit, M. Timko, The effect of long-term storage orbit on a bipropellant pressure regulation system, AIAA 93-2514, 1993.

[7] H.C. Hearn, Development and validation of fluid/thermodynamic models for spacecraft propulsion systems, Journal of Propulsion and Power 17(3) (2001) 527-533.

[8] G.P. Purohit, R.P. Prickett, Modeling of the Intelsat VI bipropellant propulsion subsystem, AIAA 93-2518, 1993. 
[9] J.M. Molinsky, Flow modeling of the Galaxy XII dual mode bipropellant propulsion subsystem, AIAA 2004-3665, 2004.

[10] I. Fletcher, C.S. Cox, W.J.B. Arden, et al, Modelling of a two-stage high-pressure gas reduction station, Applied Mathematical Modelling 20(10) (1996) 741-749.

[11] C. Fang, J.Q Li, L.F. Xu, et al, Model-based fuel pressure regulation algorithm for a hydrogen-injected PEM fuel cell engine, International Journal of Hydrogen Energy 40(43) (2015) 14942-14951.

[12] X.M. Zhang, L.H. Zhang, G.M. Jin, et al, Numerical simulation of the dynamic process for pressure regulator, Journal of Aerospace Power 19(4) (2004) 541-545. (in Chinese)

[13] J.G. Tan, Y.P. Jiang, Z.G. Wang, Instability characters and suppression method of a pressure regulator, Journal of the Brazilian Society of Mechanical Sciences and Engineering 35(1) (2013) 1-10.

[14] A Zanj, H.H. Afshari, Dynamic analysis of a complex pneumatic valve using pseudobond graph modeling technique, Journal of Dynamic Systems, Measurement, and Control 135(3) (2013) 1-9.

[15] N. Zafer, G.R. Luecke, Stability of gas pressure regulators, Applied Mathematical Modeling 32(1) (2008) 61-82.

[16] S. Sunil, P. Ullekh, B.S. Jeevanlal, et al, Mathematical modelling of pressure regulator for cryogenic applications, 37th National \& 4th International Conference on Fluid Mechanics and Fluid Power, IIT Madras, Chennai, India, 2010.

[17] E.G. Rami, B. Jean-Jacques, D. Bruno, et al, Modelling of a pressure regulator, International Journal of Pressure Vessels and Piping 84(4) (2007) 234-243.

[18] E.G. Rami, B. Jean-Jacques, G. Pascal, et al, Stability study and modelling of a pressure regulating station, International Journal of Pressure Vessels and Piping 82(1) (2005) 51-60.

[19] C.H. Shin, J.M. Ha, C.G. Lee, Transient pressure characteristics in a pressure regulating system by using 1-D analytic valve modeling, Journal of Mechanical Science and Technology 22 (2008) 374-381.

[20] C.H. Shin, A numerical study on the characteristics of transient flow in a pressure regulator resulting from closure of the pressure control valve, Journal of Mechanical Science and Technology 27(2) (2013) 443-449.

[21] K. Suzuki, I. Nakamura, J.U. Thoma, Pressure regulator valve by bondgraph, Simulation Practice and Theory 7(6) (1999) 603-611.

[22] K. Dasgupta, J. Watton, Dynamic analysis of proportional solenoid controlled piloted relief valve by bondgraph, Simulation Modelling Practice and Theory 13(1) (2005) 21-38.

[23] H.H. Afshari, A. Zanj, A.B. Novinzadeh, Dynamic analysis of a nonlinear pressure regulator using bondgraph simulation technique, Simulation Modelling Practice and Theory 18(2) (2010) 240-252.

[24] K.L. McElhaney, An analysis of check valve performance characteristics based on valve design, Nuclear Engineering and Design 197(1-2) (2000) 169-182.

[25] H. Xu, Z.M. Guang, Y.Y. Qi, Hydrodynamic characterization and optimization of Contra-push check valve by numerical simulation, Annals of Nuclear Energy 38(6) (2011) 1427-1437.

[26] H.G. Lim, J.H. Park, S.C. Jiang, Development of a swing check valve model for a low velocity pipe flow prediction, Nuclear Engineering and Design 236(10) (2006) 1051-1060.

[27] K.K. Botros, Spring stiffness selection criteria for nozzle check valves employed in compressor stations, Journal of Engineering for Gas Turbines and Power 133(12) (2011) 122401-1-11.

[28] J.P. Yu, S.R. Yu, Numerical and experimental research of flow and sound fields in an axial-flow check valve and its optimization, Advances in Mechanical Engineering 7(11) (2015) 1-8.

[29] H. Mahgerefteh, P. Saha, I.G. Economou, A study of the dynamic response of emergency shutdown valves following full bore rupture of gas pipelines, Process Safety and Environmental Protection 75(4) (1997) 201-209.

[30] S.W. Wang, Z.J. Ma, D.C. Gao, Performance enhancement of a complex chilled water system using a check valve: Experimental validation, Applied Thermal Engineering 30(17) (2010) 2827-2832.

[31] Z.J. Ma, S.W. Wang, Enhancing the performance of large primary-secondary chilled water systems by using bypass check valve, Energy 36(1) (2011) 268-276.

[32] A. Cacabelos, P. Eguía, J.L. Míguez, et al, Calibrated simulation of a public library HVAC system with a ground-source heat pump and a radiant floor using TRNSYS and GenOpt, Energy and Buildings 108(1) (2015) 114-126.

[33] E. Topriska, M. Kolokotroni, Z. Dehouche, et al, Solar hydrogen system for cooking applications: Experimental and numerical study, Renewable Energy 83 (2015) 717-728.

[34] Thermal Energy Systems Specialists, TRNSYS (A Transient Simulation Program) 17.1 Manuals, 2012, available at: www.trnsys.com and http://sel.me.wisc.edu/trnsys.

[35] J.K. Kang, G.W. Auner, Simulation and verification of a piezoelectrically actuated diaphragm for check valve micropump design, Sensors and Actuators A: Physical 167(2) (2011) 512-516.

[36] K. Ou, J. Jackson, H. Burt, et al, Microspheres as resistive elements in a check valve for low pressure and low flow rate conditions, Lab on a Chip 12(21) (2012) 4372-4380.

[37] Y. Chen, G.B. Cai, Z.P. Zhang, et al, Multi-field coupling dynamic modeling and simulation of turbine test rig gas system, Simulation Modelling Practice and Theory 44 (2014) 95-118.

[38] Y. Chen, G.B. Cai, Z. Wu, Modularization modeling and simulation of turbine test rig main test system, Applied Mathematical Modelling 35(11) (2011) 5382-5399.

[39] Y. Chen, G.B. Cai, Z.P. Zhang, et al, Numerical simulation on dynamic stability of pressure reducing regulator in integral bipropellant propulsion system, Journal of Beijing University of Aeronautics and Astronautics 36(10) (2010) 1135-1139. (in Chinese)

[40] J.M. Saleh, Fluid Flow Handbook, New York: McGraw-Hill, 2002.

[41] Y.Z. Zhang, P. Wang, Z.X. Han, et al, Fluid Dynamics Handbook, Beijing: Beijing University of Aeronautics and Astronautics Press, 1991. (in Chinese)

[42] W.M. Rohsenow, J.P. Hartnett, Y.I. Cho, Handbook of Heat Transfer (3rd Edition), New York: McGraw-Hill, 1998. 
[43] J.H. Lienhard IV, J.H. Lienhard V, A Heat Transfer Textbook (Fourth Edition), Cambridge, Massachusetts: Phlogiston Press, 2016.

[44] B. Sun, Q. Xu, Y. Chen, et al, Numerical analysis of influence factors on stability for dual-stage gas pressure reducing regulator, Journal of Beijing University of Aeronautics and Astronautics 40(12) (2014) 1660-1665. (in Chinese)

[45] Y. Chen, Z.P. Zhang, S.F. Yang, et al, Distributed parameter numerical simulation for the transients of cryogenic propellant tank pressurization system (II) Numerical model and simulation results of pressurization system, Journal of Aerospace Power 23(2) (2008) 329-335. (in Chinese)

[46] N.N. Smirnov, V.B. Betelin, V.F. Nikitin, et al, Accumulation of errors in numerical simulations of chemically reacting gas dynamics, Acta Astronautica 117 (2015) 338-355.

[47] S. Kawai, S.K. Lele, Localized artificial diffusivity scheme for discontinuity capturing on curvilinear meshes, Journal of Computational Physics 227 (2008) 9498-9526.

[48] A.K. Majumdar, Generalized Fluid System Simulation Program, Version 5.0-Educational, NASA/TM-2011-216470, 2011.

[49] A. Bandyopadhyay, A. Majumdar, Network flow simulation of fluid transients in rocket propulsion systems, Journal of Propulsion and Power 30(6) (2014) 1646-1653. 


\section{Illustrations}

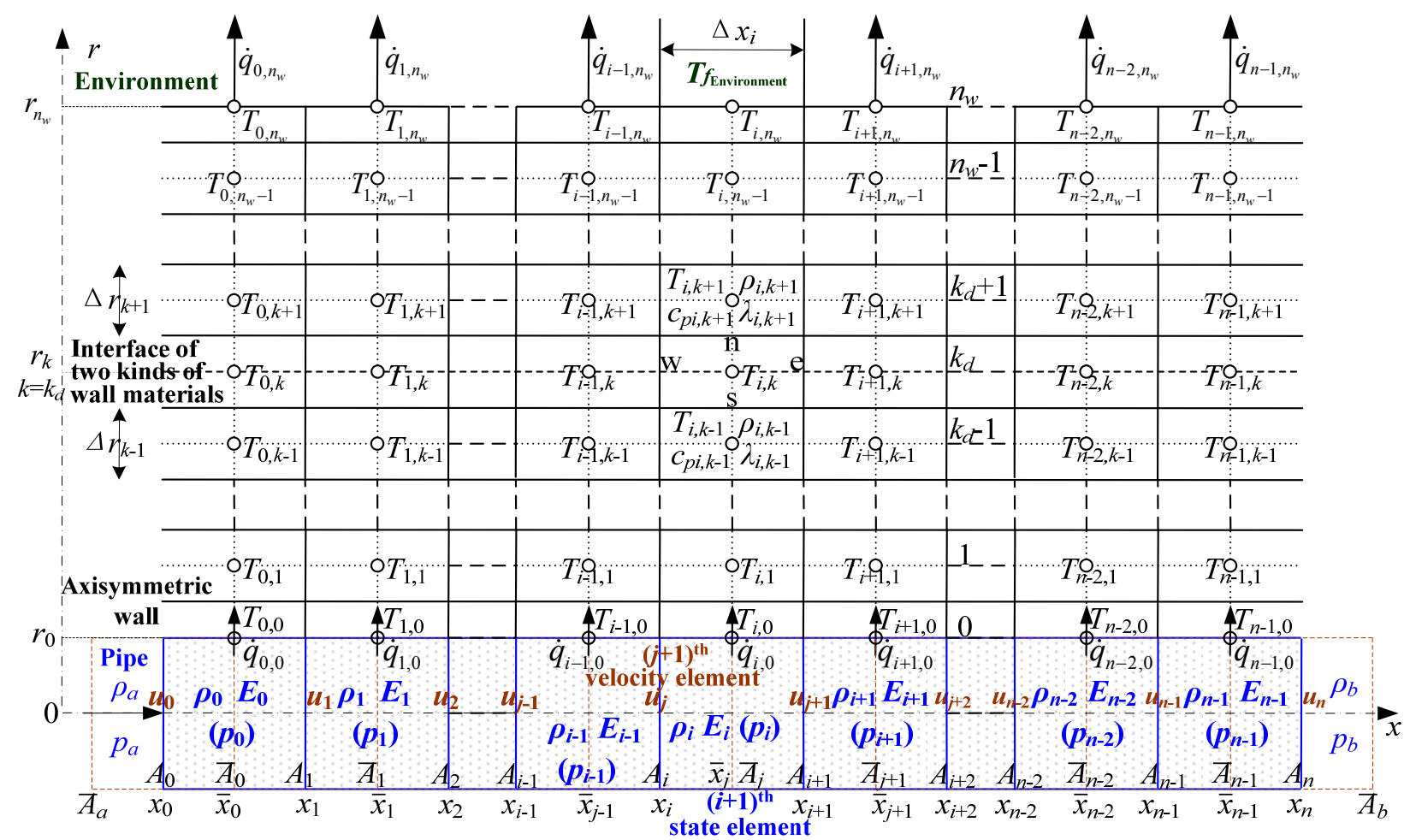

Fig. 1 Finite control volume grids of 1D compressible transient pipe flow and 2D axisymmetric wall temperature field

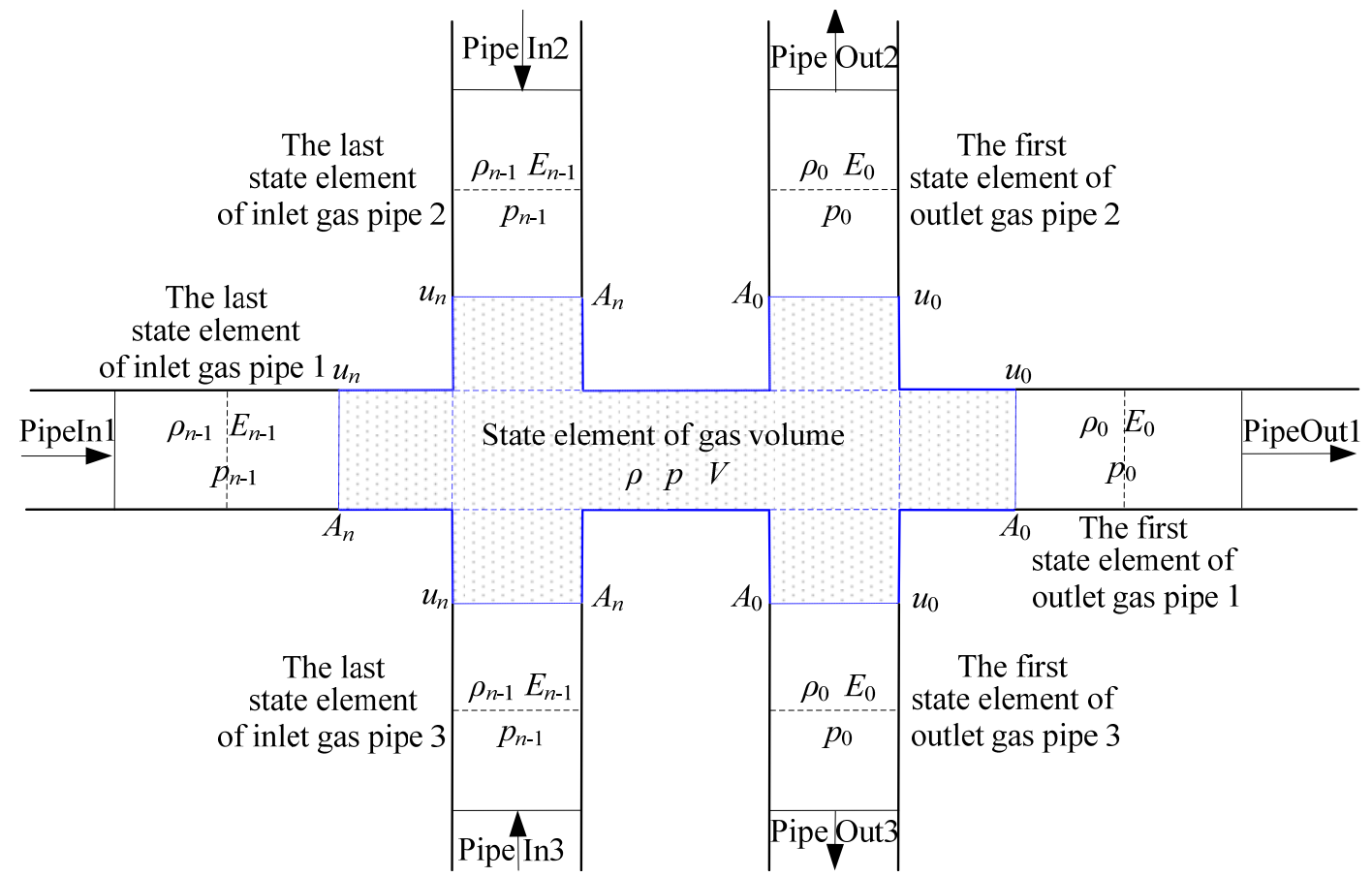

Fig. 2 Finite control volume grid of gas volume 


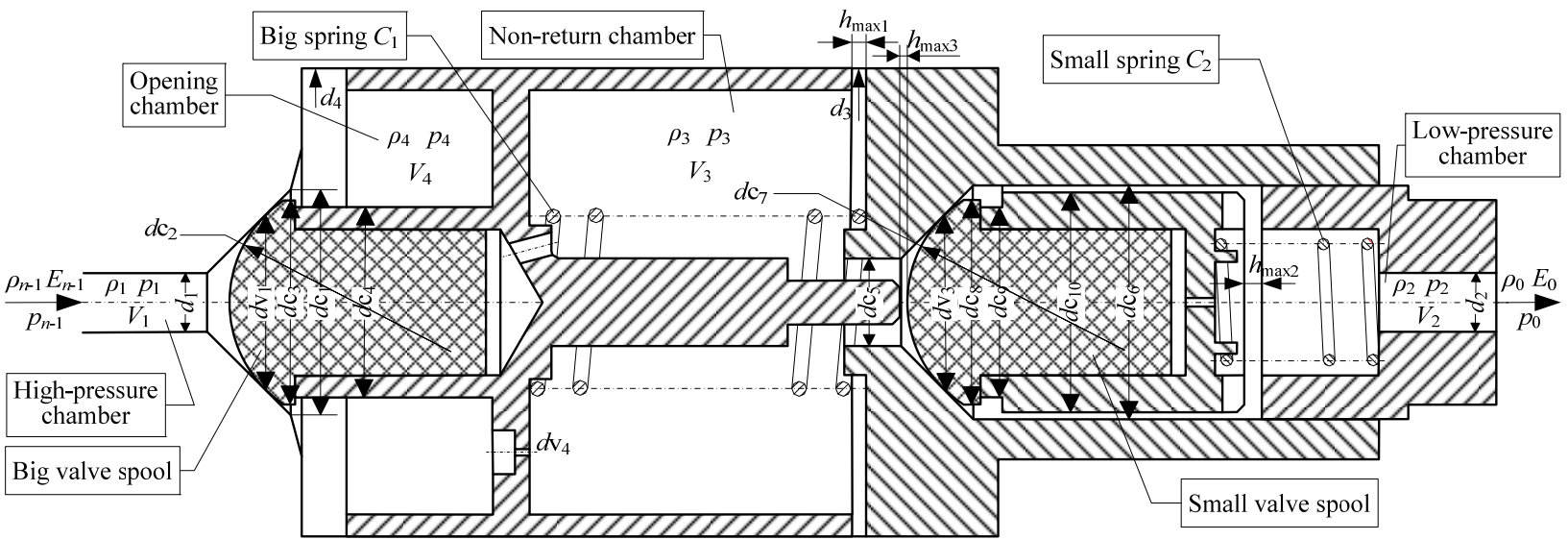

Fig. 3 Schematic of the double-spool lift check valve

The last

state element High-pressure Opening of gas pipe $u_{n}$ chamber $u_{1} u_{4}$ chamber

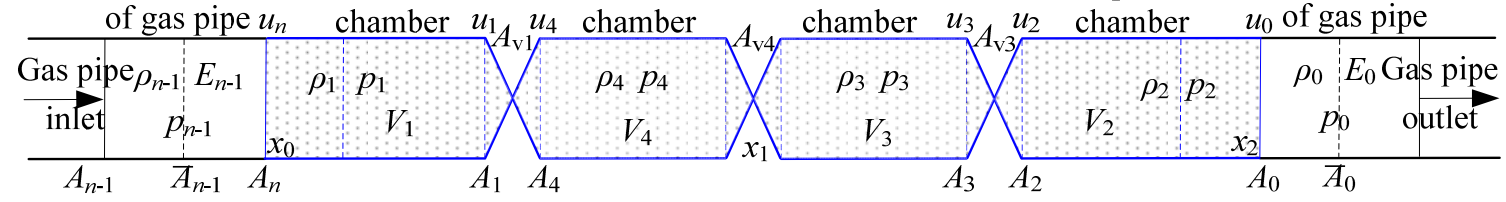

Fig. 4 Finite control volume grids of the double-spool lift check valve

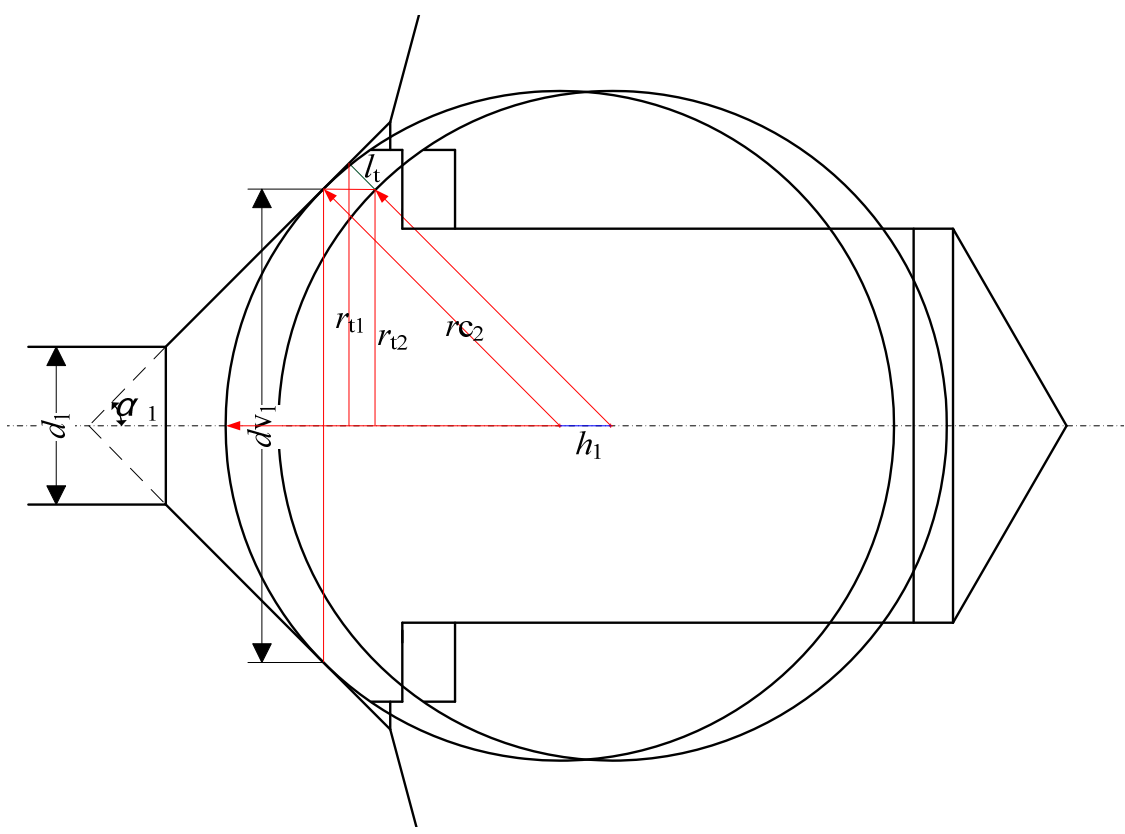

Fig. 5 Schematic of big valve spool throttling of the double-spool lift check valve 


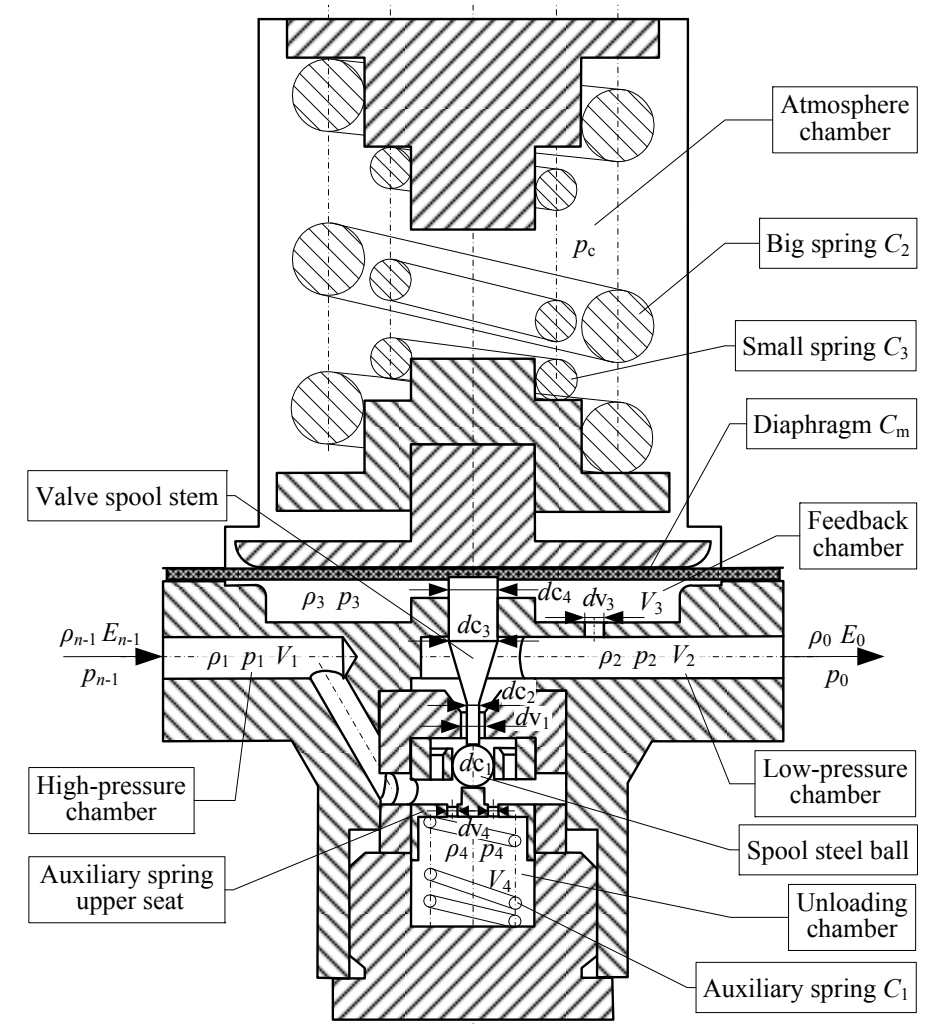

Fig. 6 Schematic of the high-pressure-unloading diaphragm-type regulator

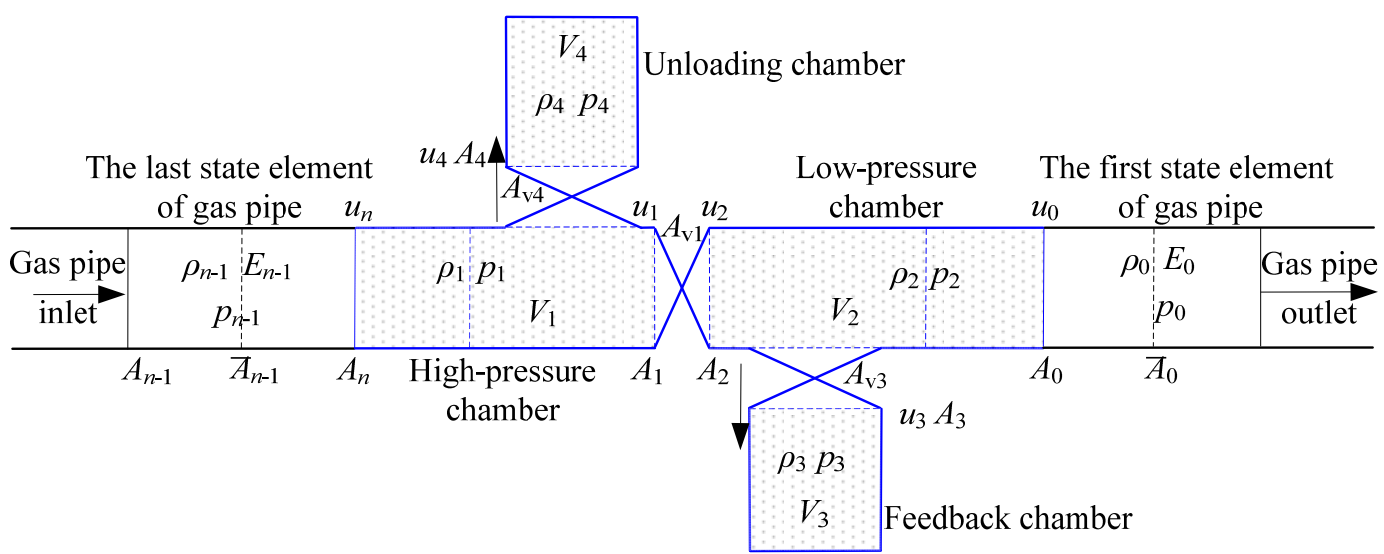

Fig. 7 Finite control volume grids of the high-pressure-unloading diaphragm-type regulator

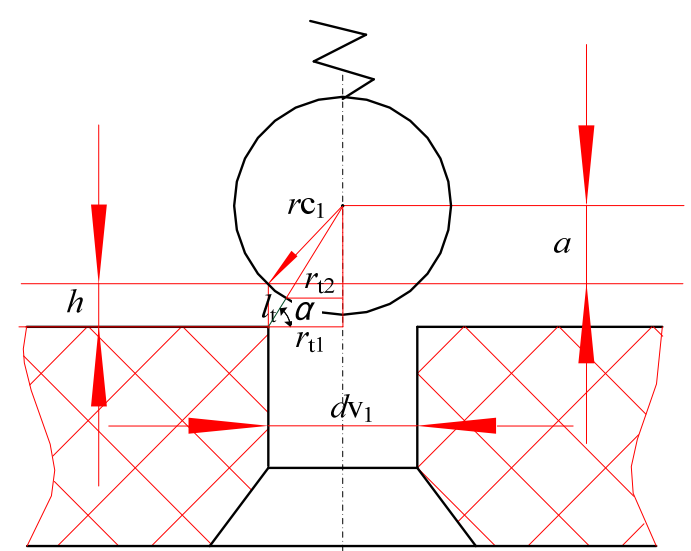

Fig. 8 Schematic of valve-spool throttling of the high-pressure-unloading diaphragm-type regulator 


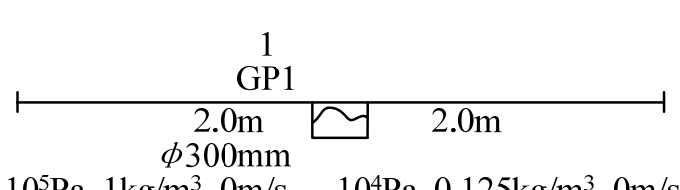

$10^{5} \mathrm{~Pa}, 1 \mathrm{~kg} / \mathrm{m}^{3}, 0 \mathrm{~m} / \mathrm{s} \quad 10^{4} \mathrm{~Pa}, 0.125 \mathrm{~kg} / \mathrm{m}^{3}, 0 \mathrm{~m} / \mathrm{s}$

(a) One-dimensional Riemann problem used by Sod

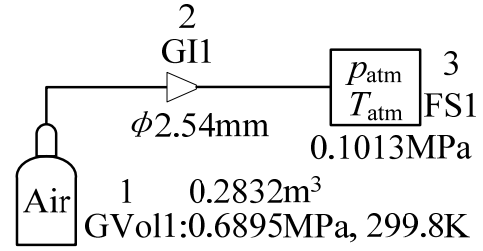

(b) Venting air tank problem used by Majumdar

Fig. 9 Numerical simulation models of (a) Sod shock tube problem and (b) Venting air tank problem
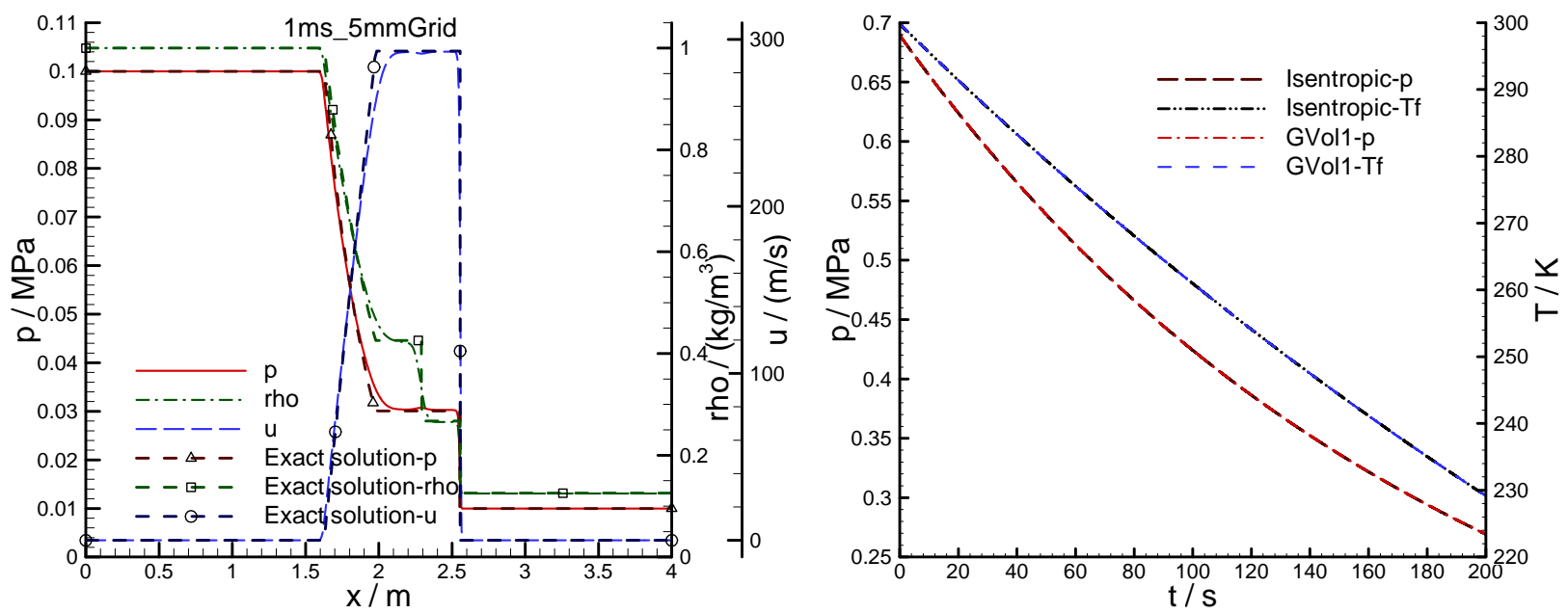

(a) Pressure, density and velocity profiles along axis at time instant $1 \mathrm{~ms}$

(b) Pressure and temperature history of air tank

Fig. 10 Comparison of simulation results and analytical solution: (a) Sod shock tube problem and (b) Venting air tank problem

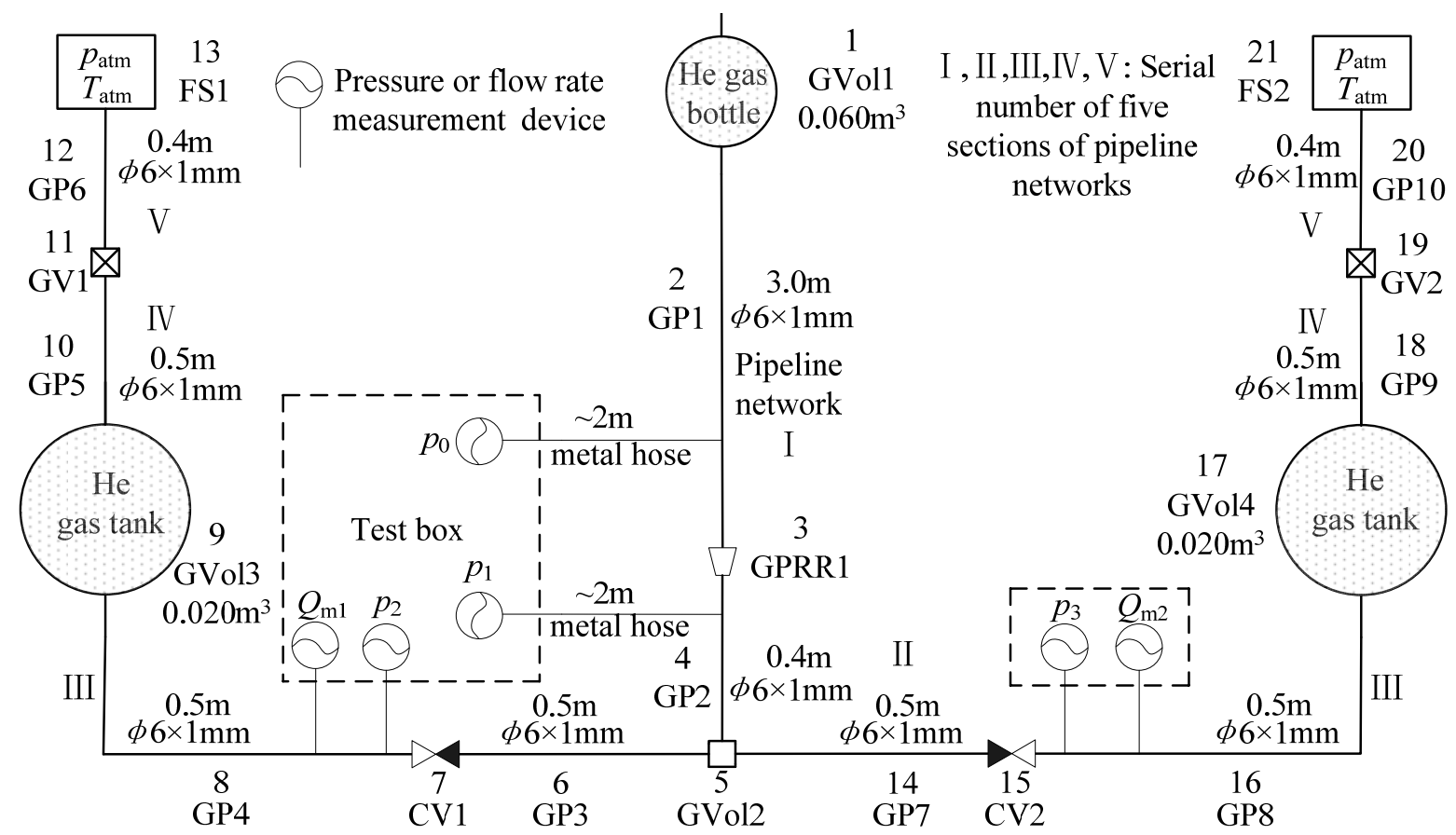

Fig. 11 Numerical simulation model of double-valve combined test system 


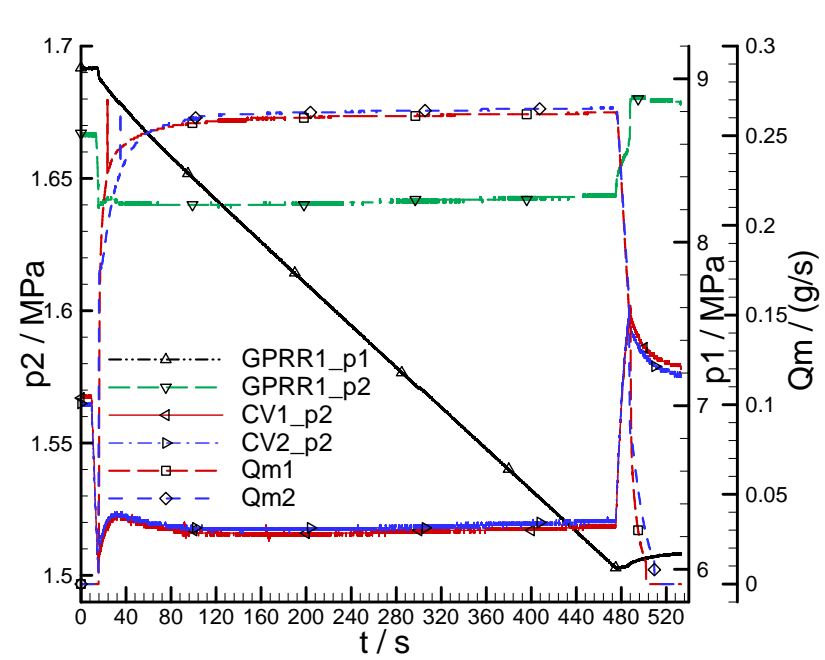

(a) The whole process of combined test

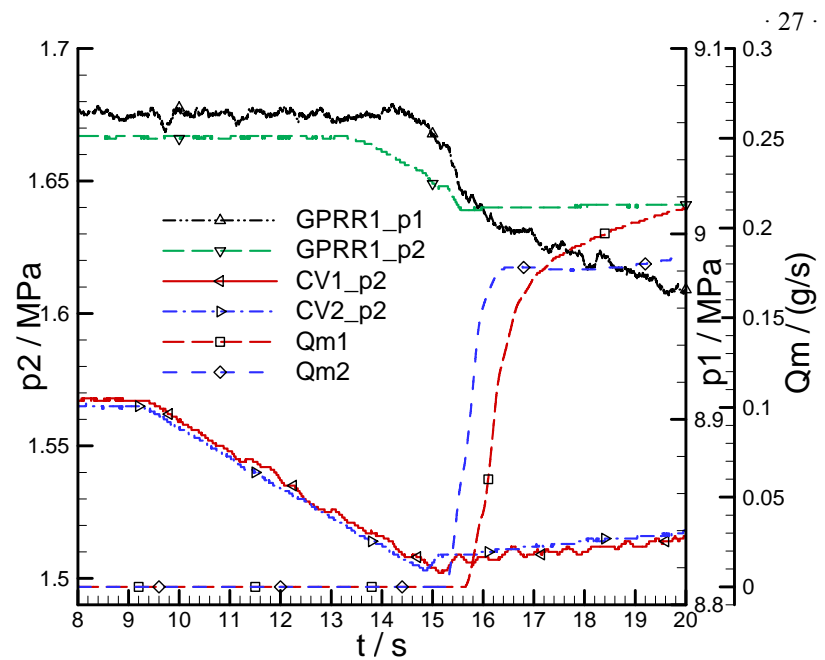

(b) The startup stage of system

Fig. 12 Experimental measured results of 9MPa working condition of double-valve combined test system

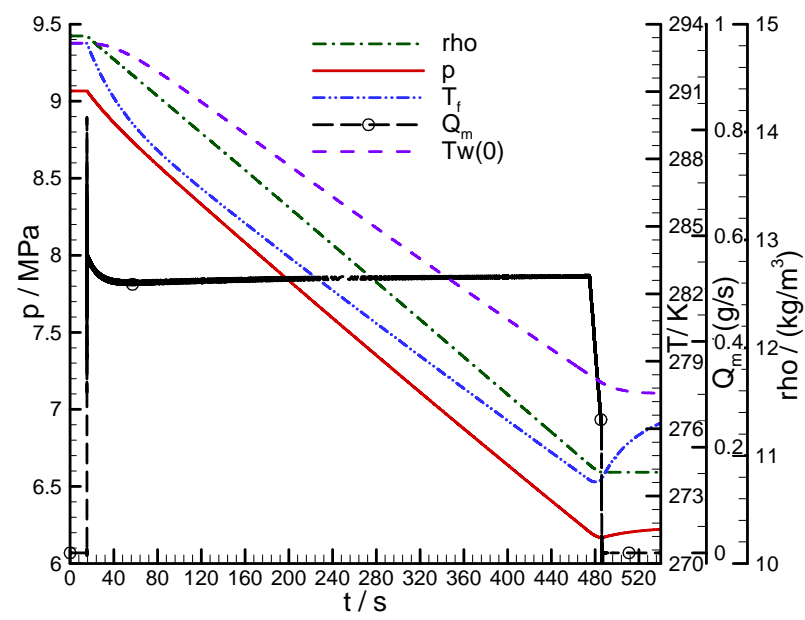

(a) GVol1

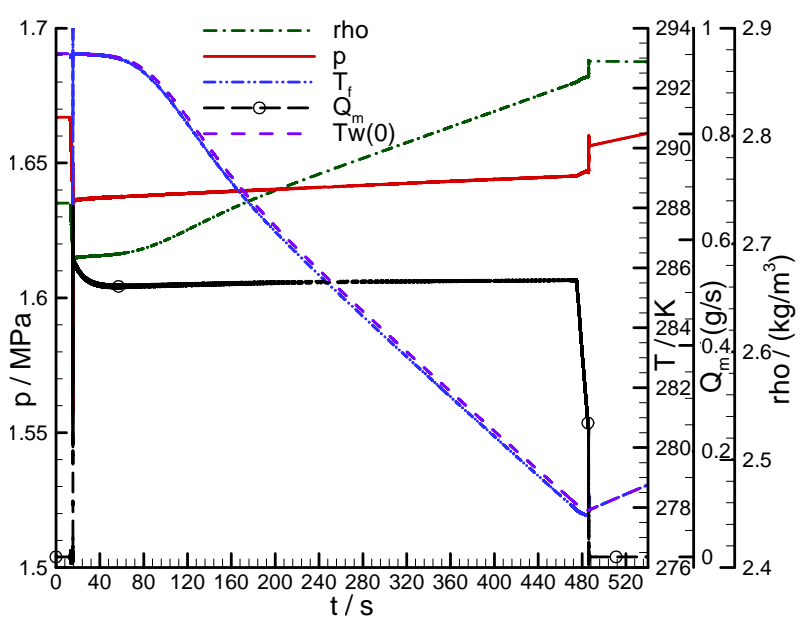

(b) GVol2

Fig. 13 Simulation results of pressures, fluid and wall temperatures, mass flow rates and densities of (a) GVoll and (b) GVol2

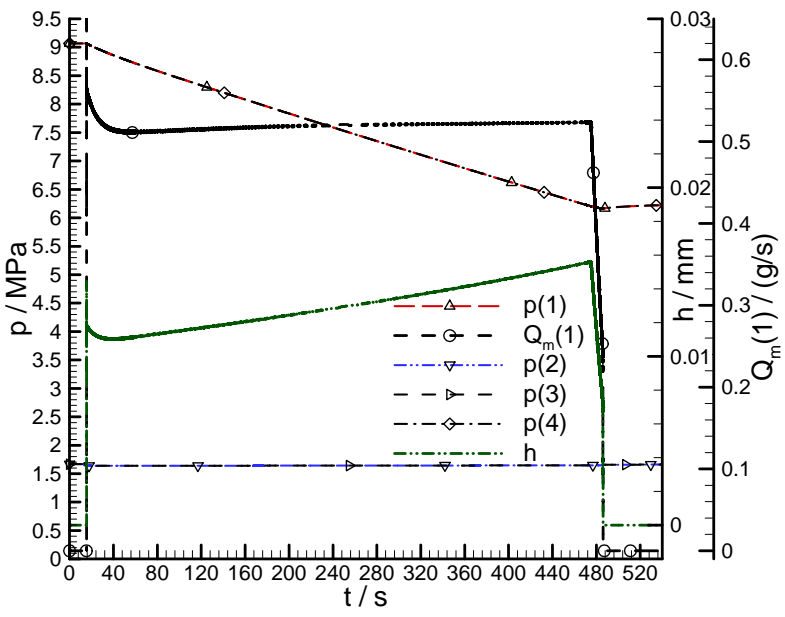

(a) Pressures of four chambers, opening of valve spool, mass flow rate at the throttling point of valve spool

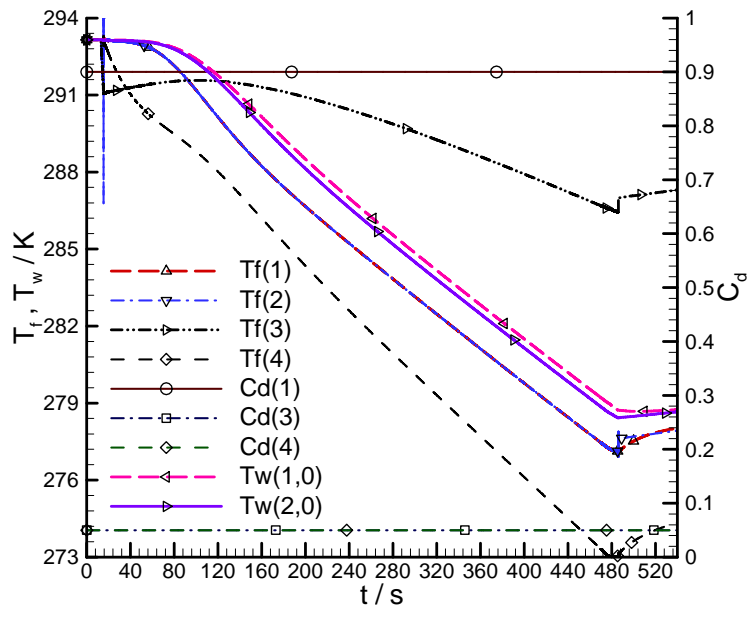

(b) Fluid temperatures of four chambers, interior wall temperatures of high-pressure and low-pressure chambers, flow coefficients of three throttling points 


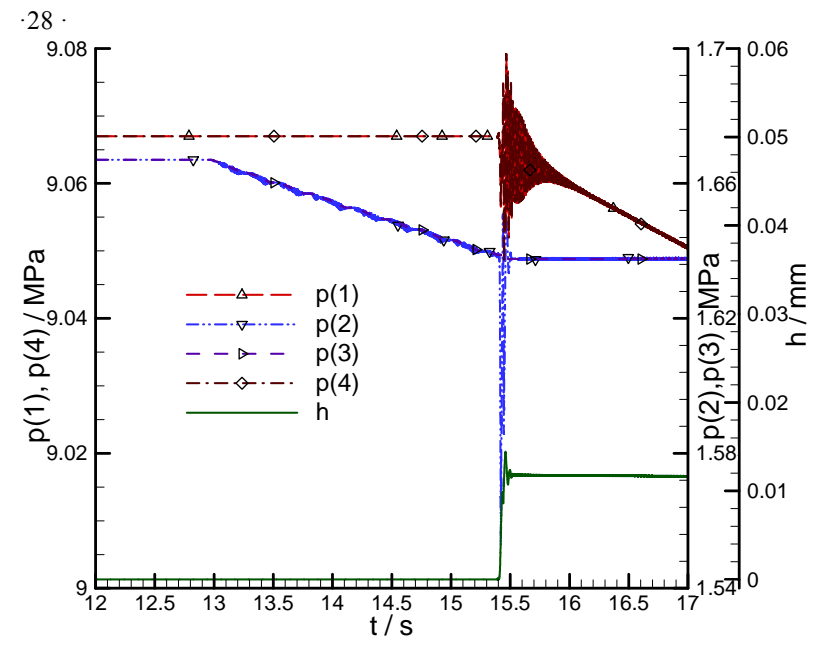

(c) Pressures of four chambers, opening of valve spool at the startup stage of system

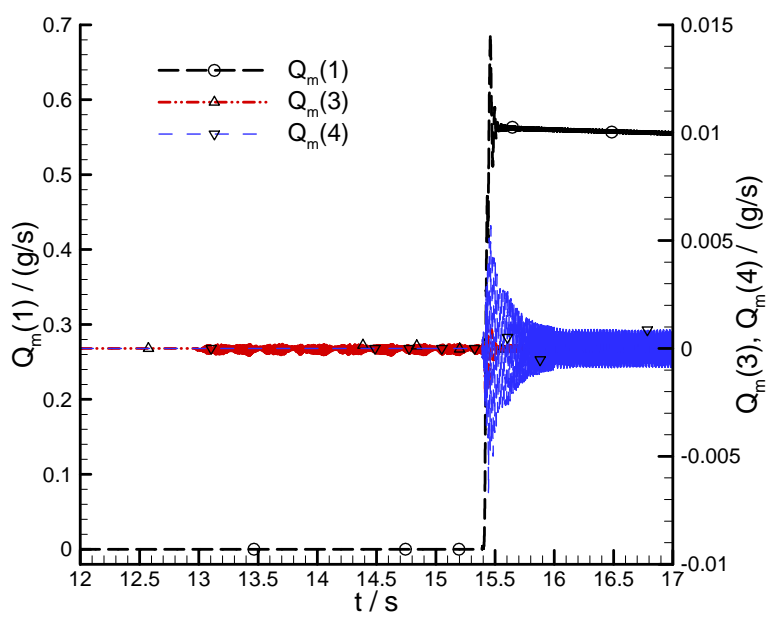

(e) Mass flow rates at three throttling points at the startup stage of system

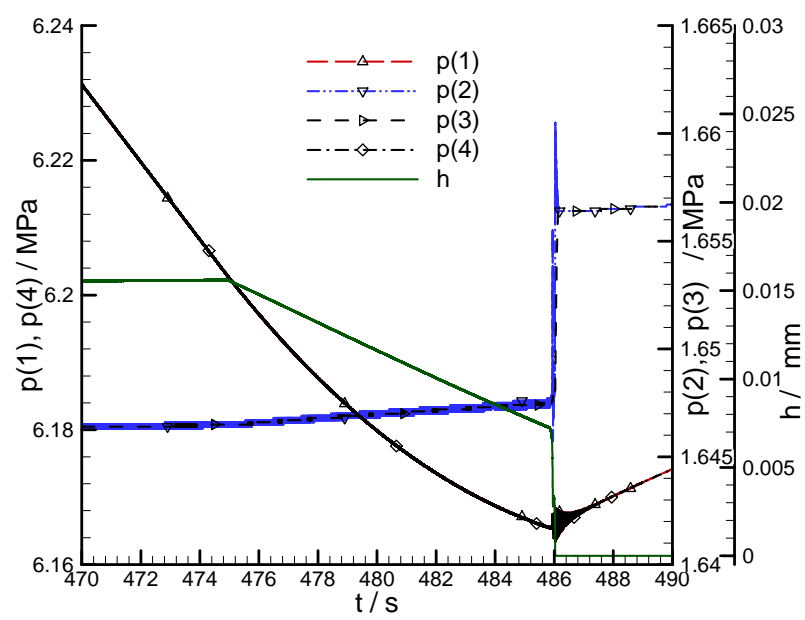

(g) Pressures of four chambers, opening of valve spool at the shutdown stage of system

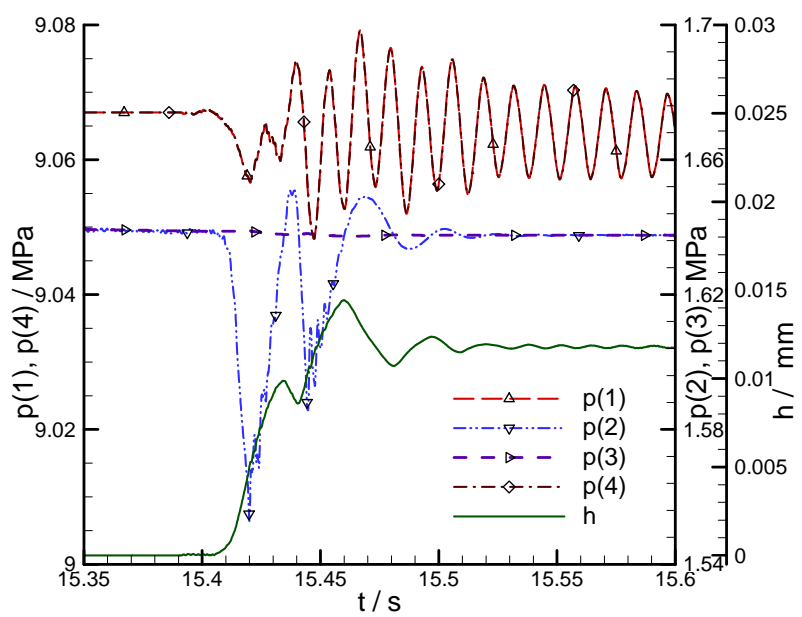

(d) Pressures of four chambers, opening of valve spool at the opening stage of GPRR1 spool

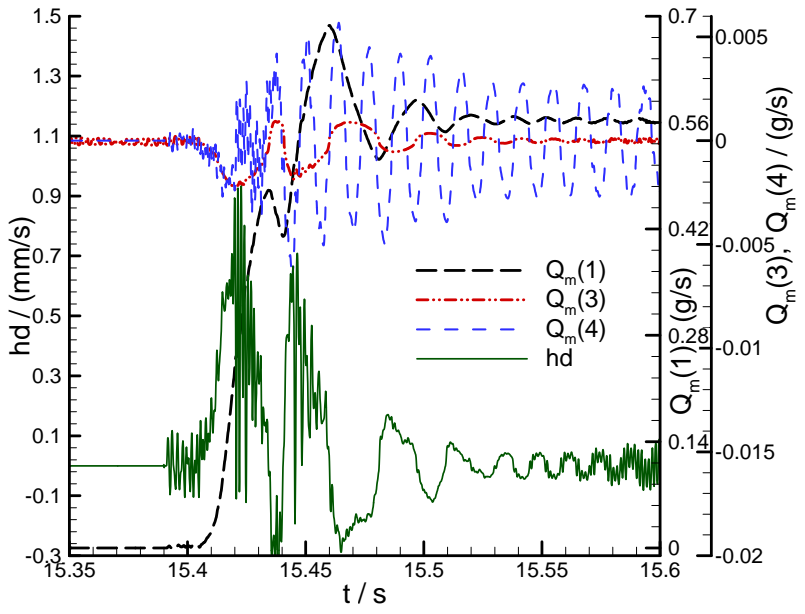

(f) Movement velocity of valve spool, mass flow rates at three throttling points at the opening stage of GPRR1 spool

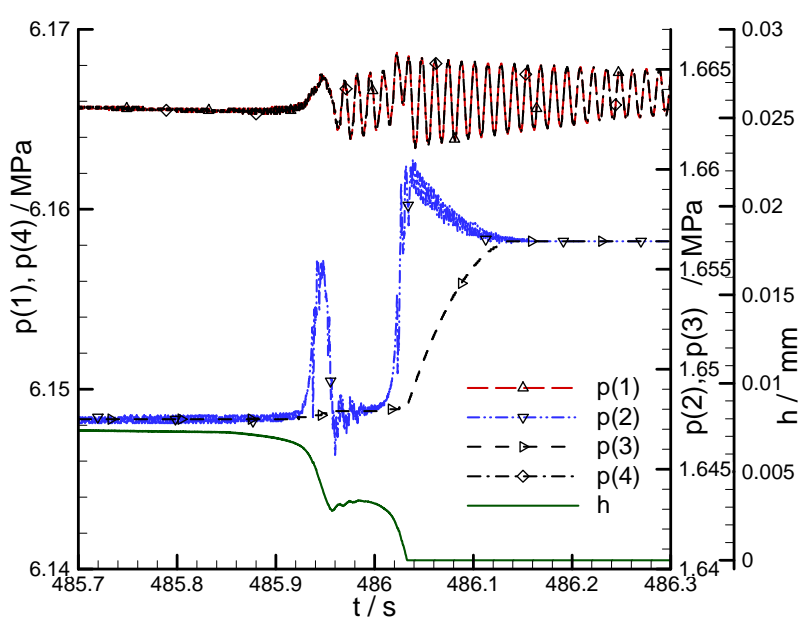

(h) Pressures of four chambers, opening of valve spool at the closing stage of GPRR1 spool 


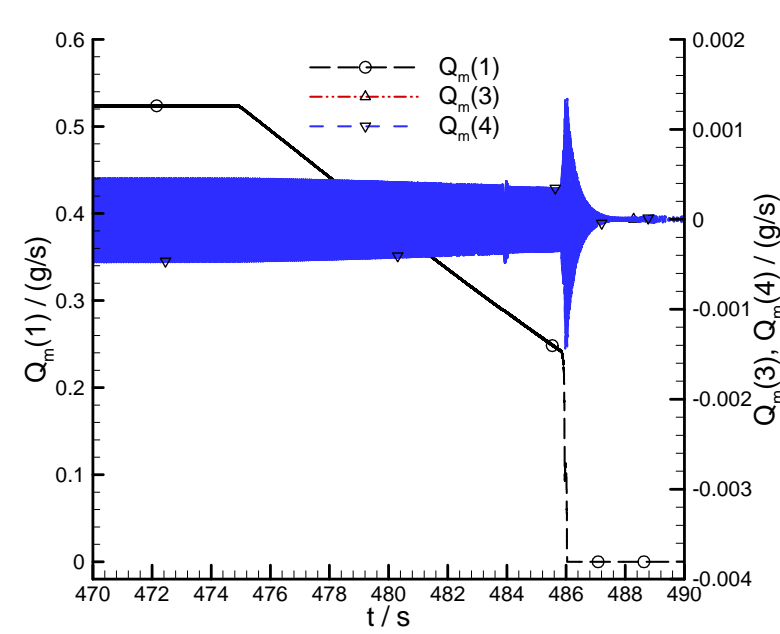

(i) Mass flow rates at three throttling points at the shutdown stage of system

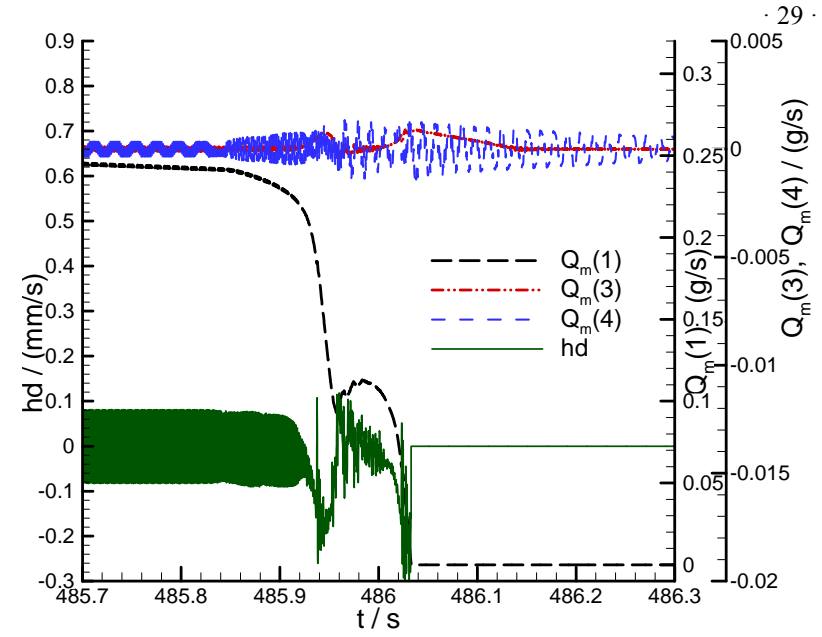

(j) Movement velocity of valve spool, mass flow rates at three throttling points at the closing stage of GPRR1 spool

Fig. 14 Simulation results at four chambers including high-pressure chamber, low-pressure chamber, feedback chamber and unloading chamber and three throttling points including valve spool, feedback orifice and unloading orifice of GPRR1

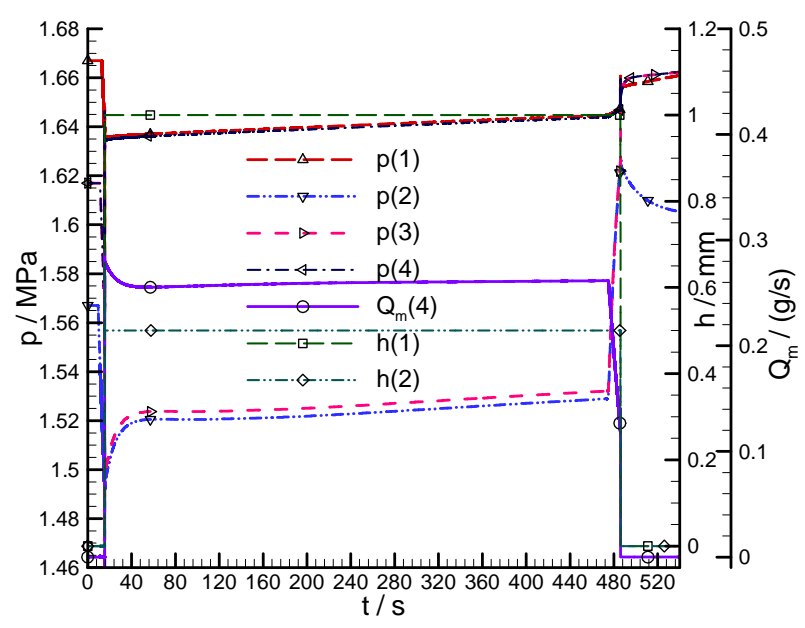

(a) Pressures of four chambers, openings of big and small valve spools, mass flow rate of non-return orifice

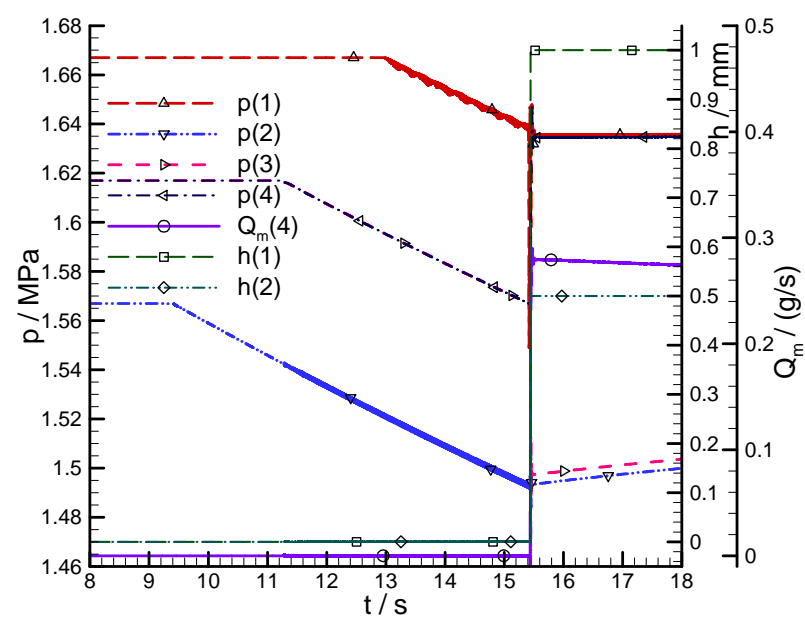

(c) Pressures of four chambers, openings of big and small valve spools, mass flow rate of non-return orifice at the startup stage of system

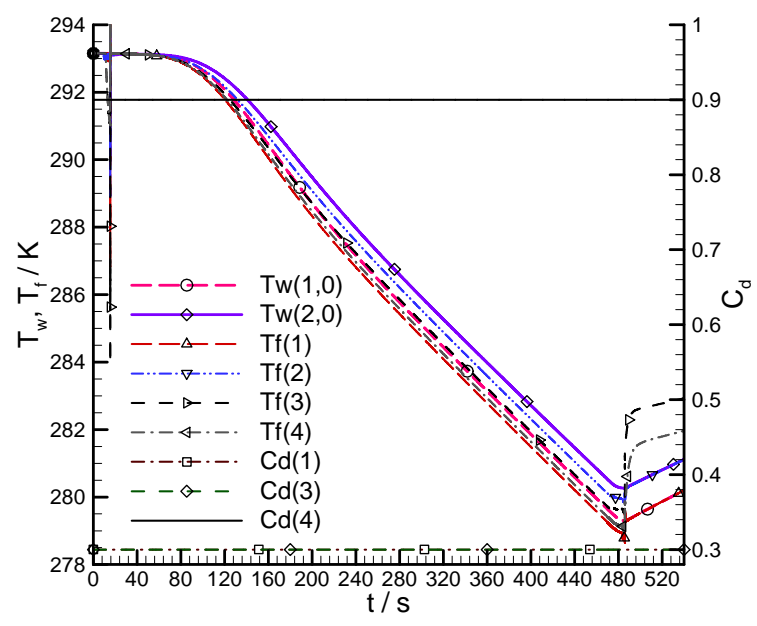

(b) Interior wall temperatures of high-pressure and low-pressure chambers, fluid temperatures of four chambers, flow coefficients of three throttling points

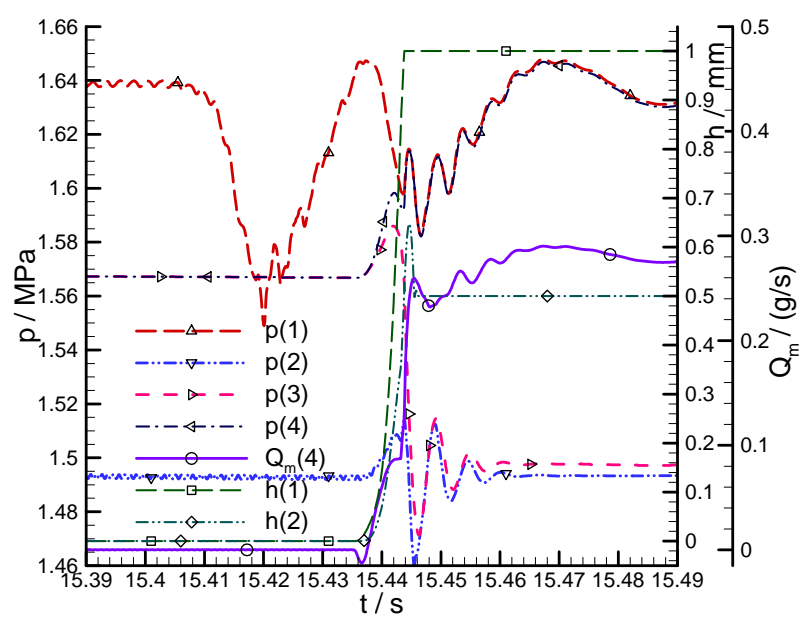

(d) Pressures of four chambers, openings of big and small valve spools, mass flow rate of non-return orifice at the opening stage of CV1 spools 
30 .

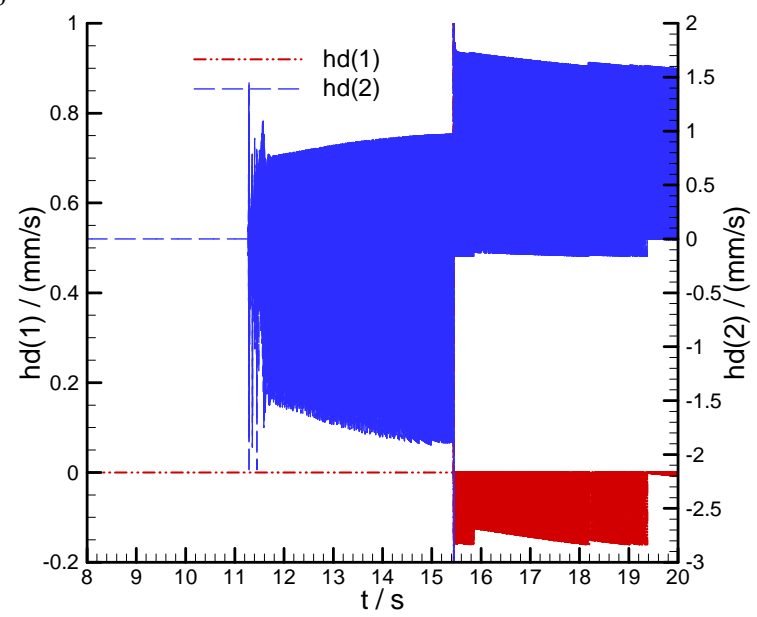

(e) Movement velocities of big and small valve spools at the startup stage of system

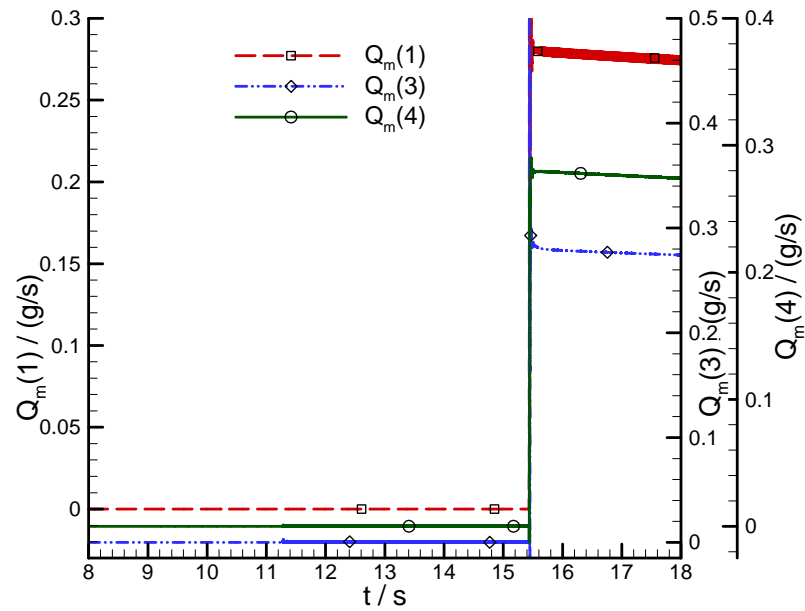

(g) Mass flow rates at three throttling points of big and small valve spools, non-return orifice at the startup stage of system

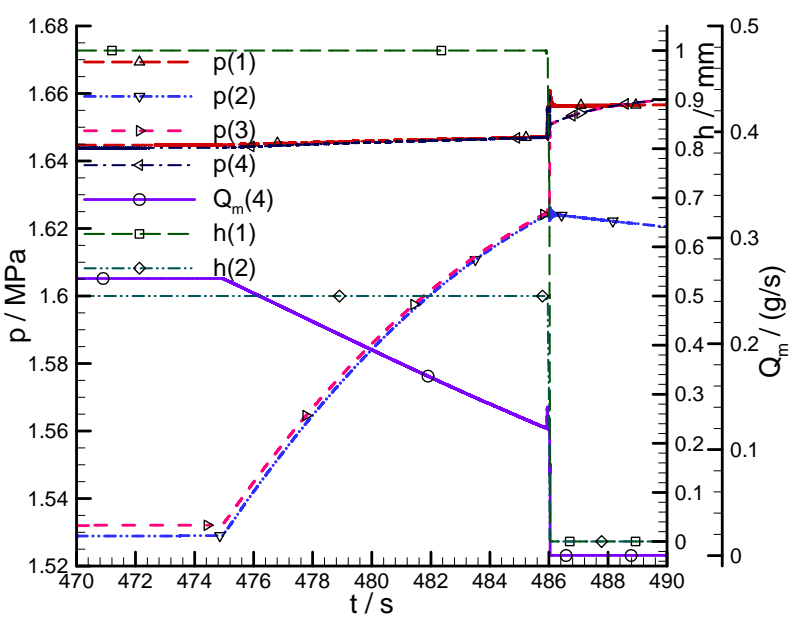

(i) Pressures of four chambers, openings of big and small valve spools, mass flow rate of non-return orifice at the shutdown stage of system

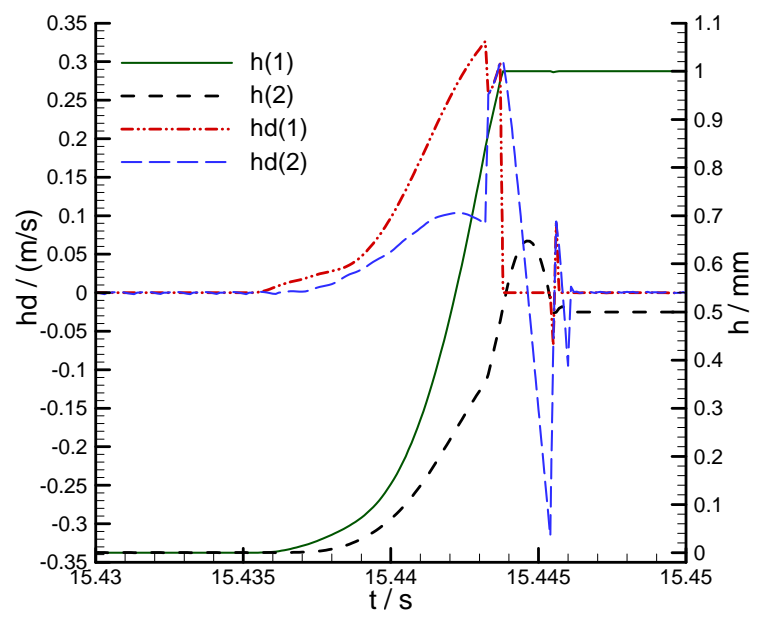

(f) Movement velocities and openings of big and small valve spools at the opening stage of CV1 spools

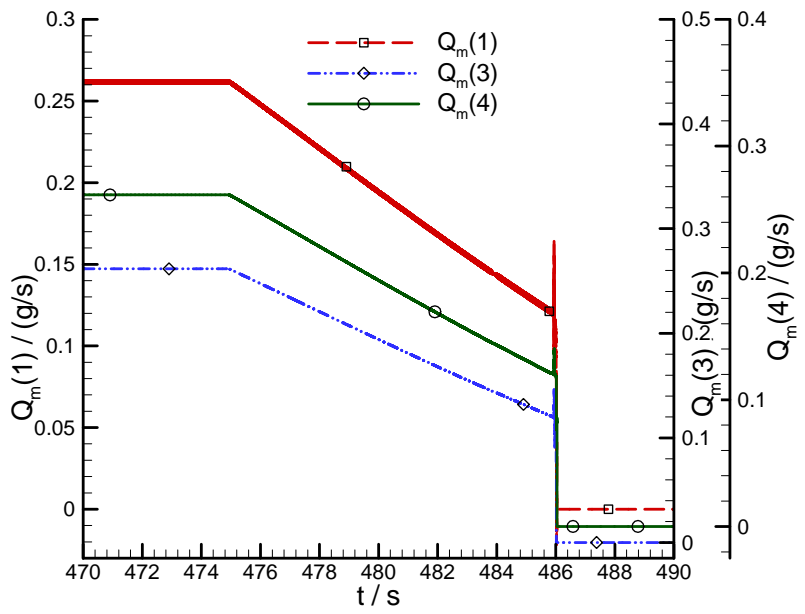

(h) Mass flow rates at three throttling points of CV1 at the shutdown stage of system

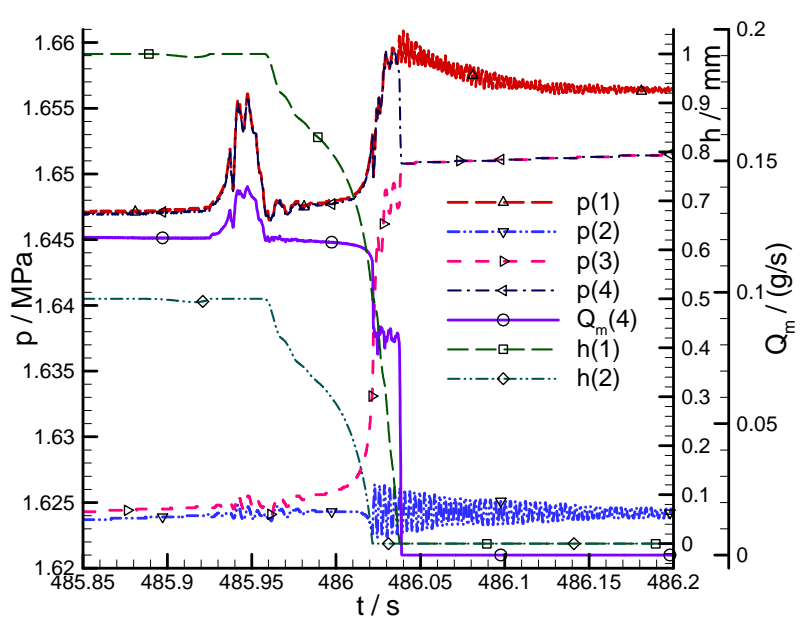

(j) Pressures of four chambers, openings of big and small valve spools, mass flow rate of non-return orifice at the closing stage of $\mathrm{CV} 1$ spools 


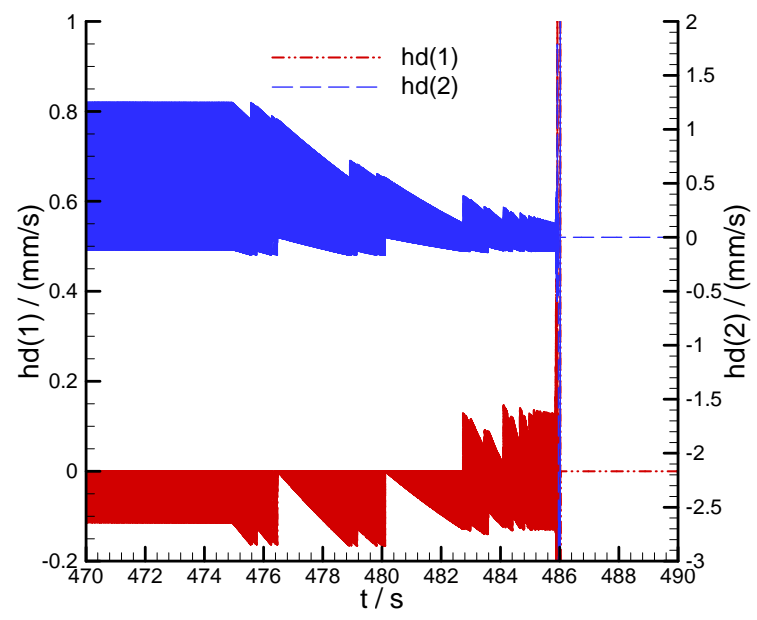

(k) Movement velocities of big and small valve spools at the shutdown stage of system

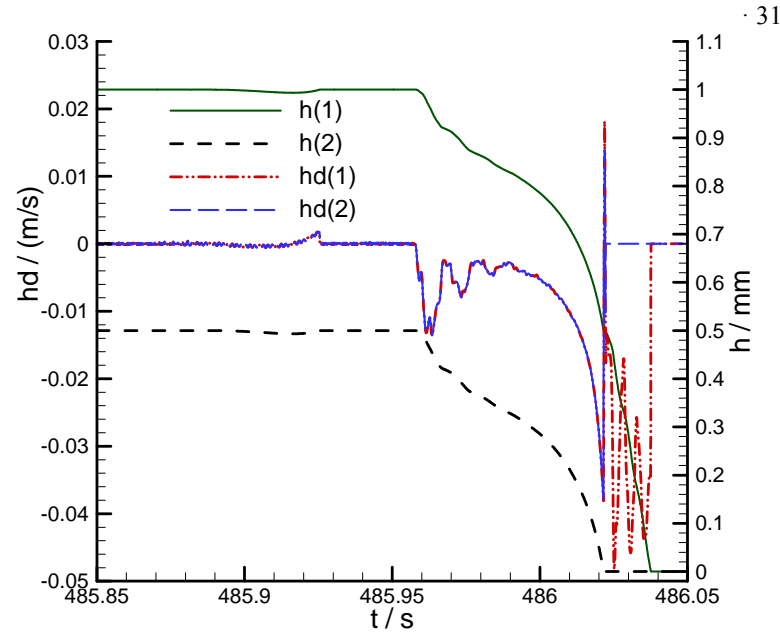

(1) Movement velocities and openings of big and small valve spools at the closing stage of CV1 spools

Fig. 15 Simulation results at four chambers including high-pressure chamber, low-pressure chamber, non-return chamber and opening chamber and three throttling points including big valve spool, small valve spool and non-return orifice of CV1

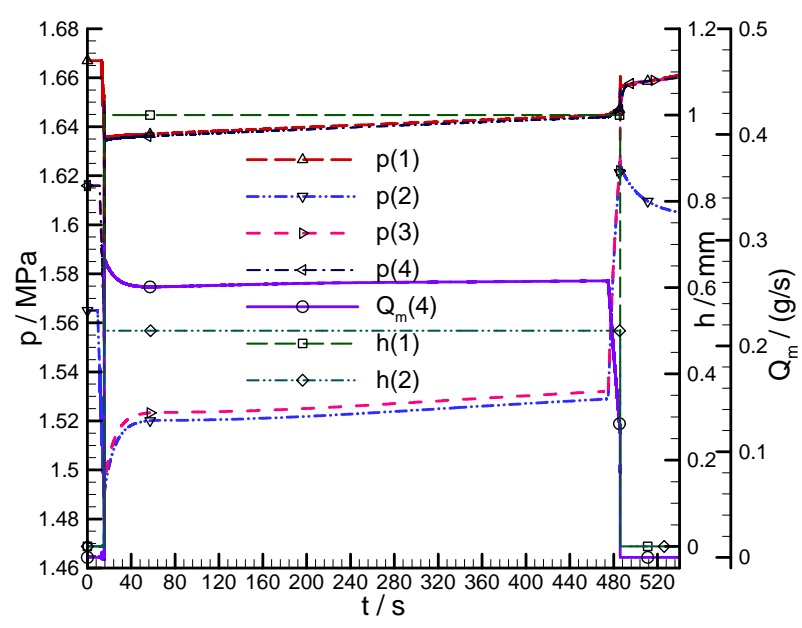

(a) Pressures of four chambers, openings of big and small valve spools, mass flow rate of non-return orifice

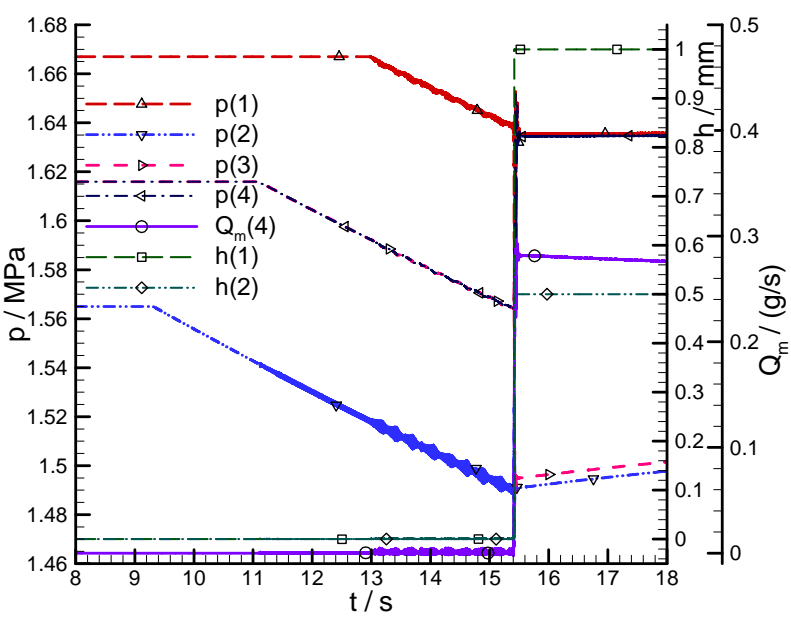

(c) Pressures of four chambers, openings of big and small valve spools, mass flow rate of non-return orifice at the startup stage of system

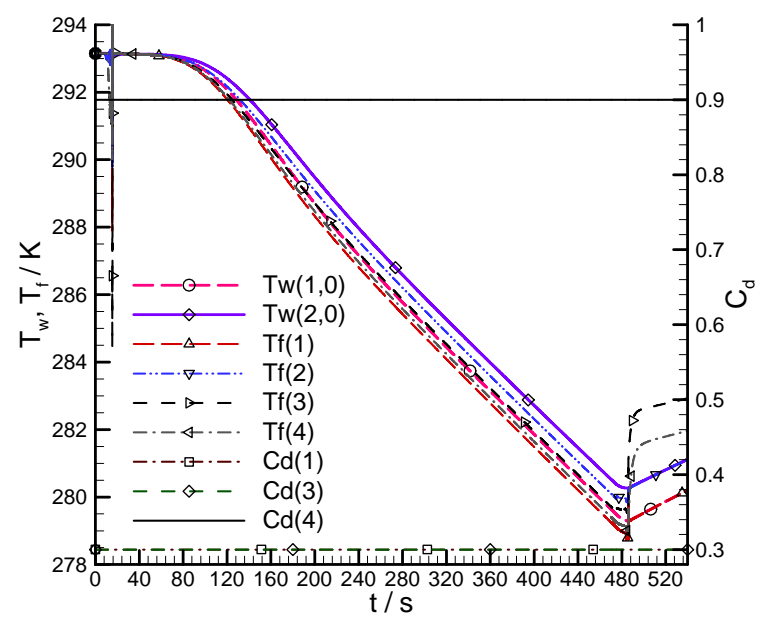

(b) Interior wall temperatures of high-pressure and low-pressure chambers, fluid temperatures of four chambers, flow coefficients of three throttling points

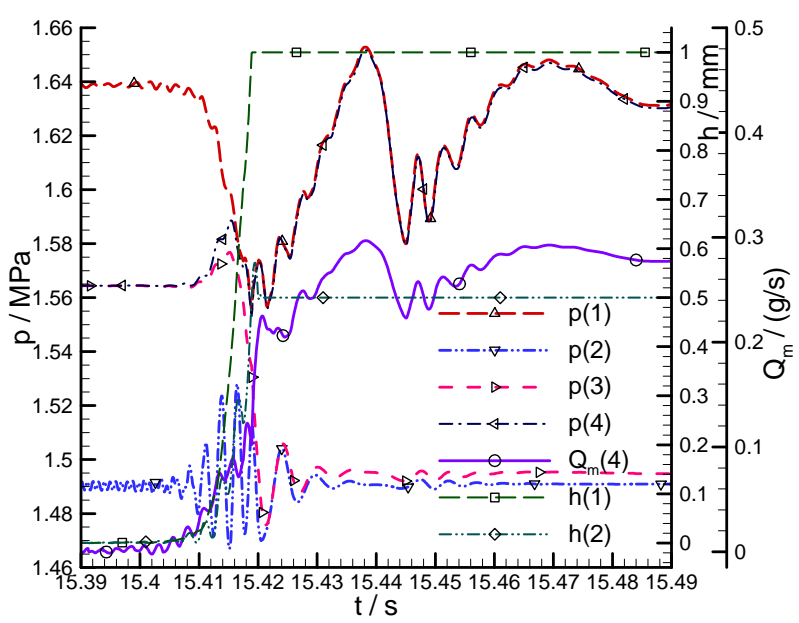

(d) Pressures of four chambers, openings of big and small valve spools, mass flow rate of non-return orifice at the opening stage of CV2 spools 


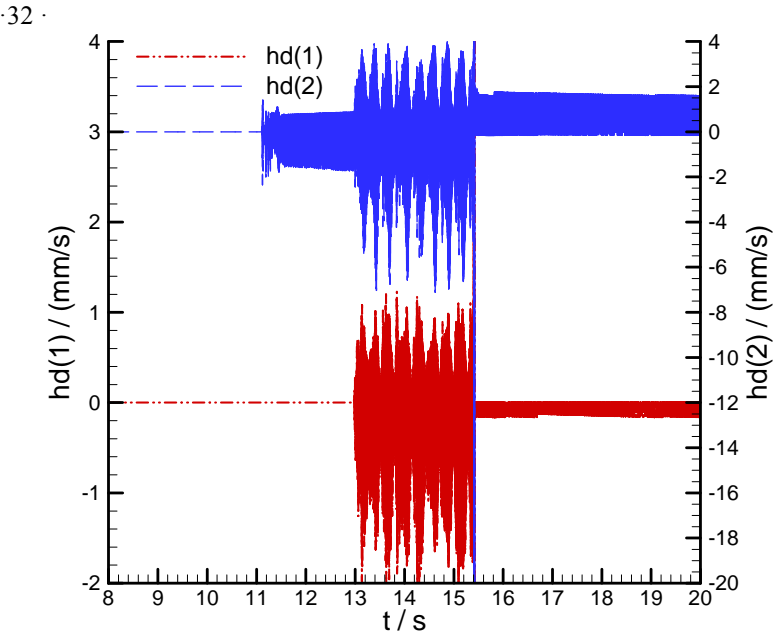

(e) Movement velocities of big and small valve spools at the startup stage of system

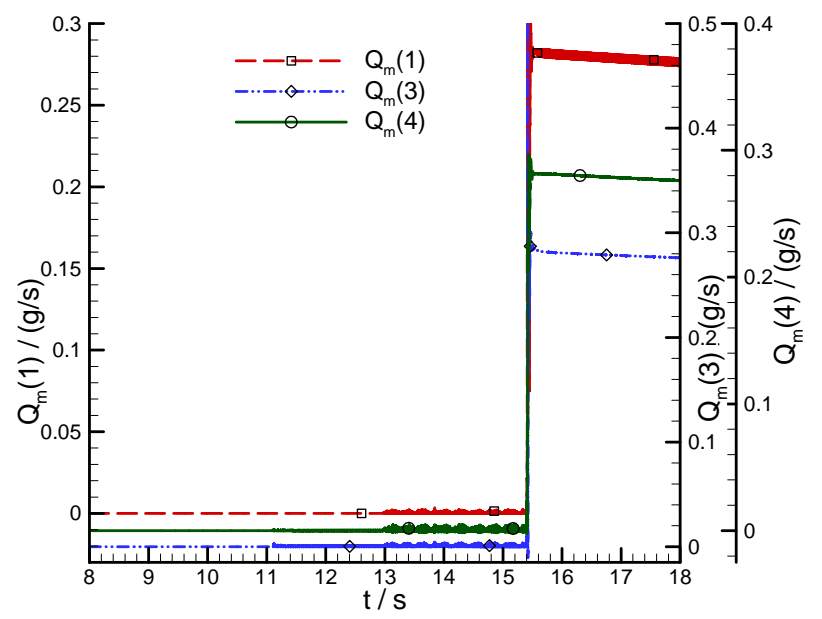

(g) Mass flow rates at three throttling points of big and small valve spools, non-return orifice at the startup stage of system

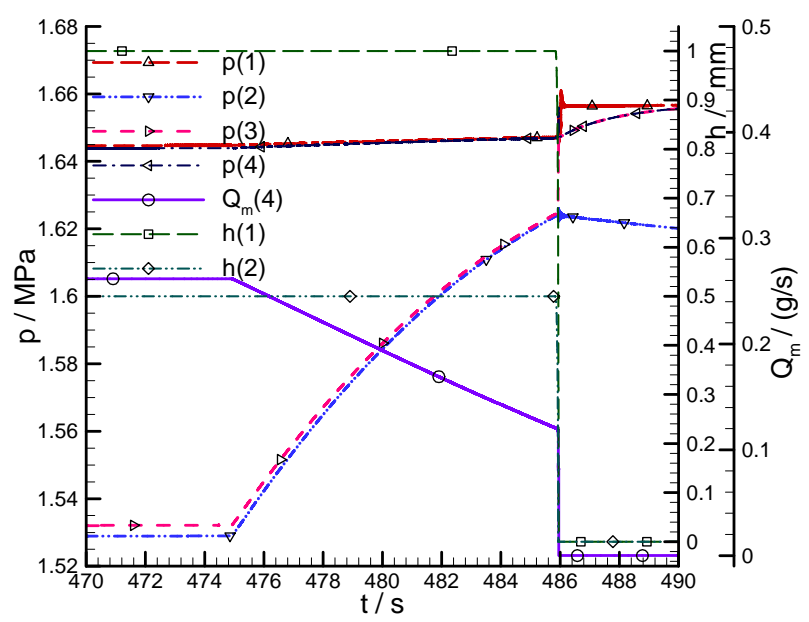

(i) Pressures of four chambers, openings of big and small valve spools, mass flow rate of non-return orifice at the shutdown stage of system

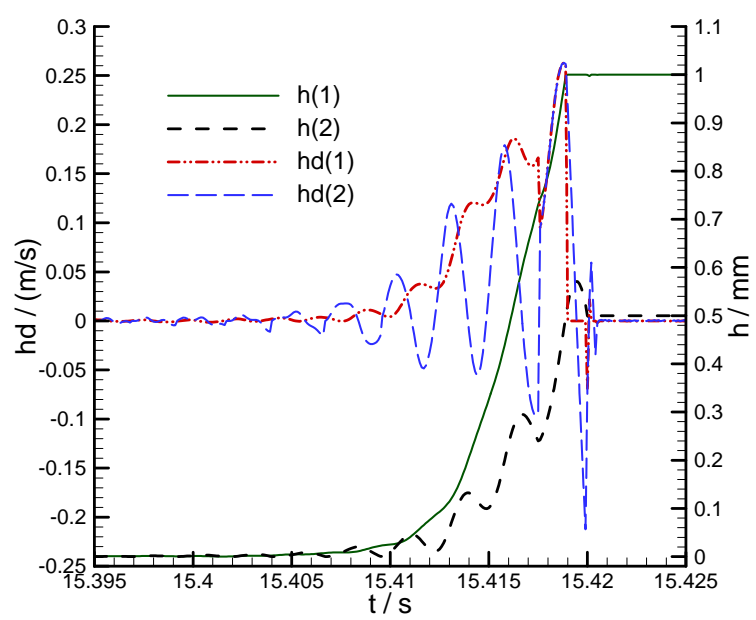

(f) Movement velocities and openings of big and small valve spools at the opening stage of CV2 spools

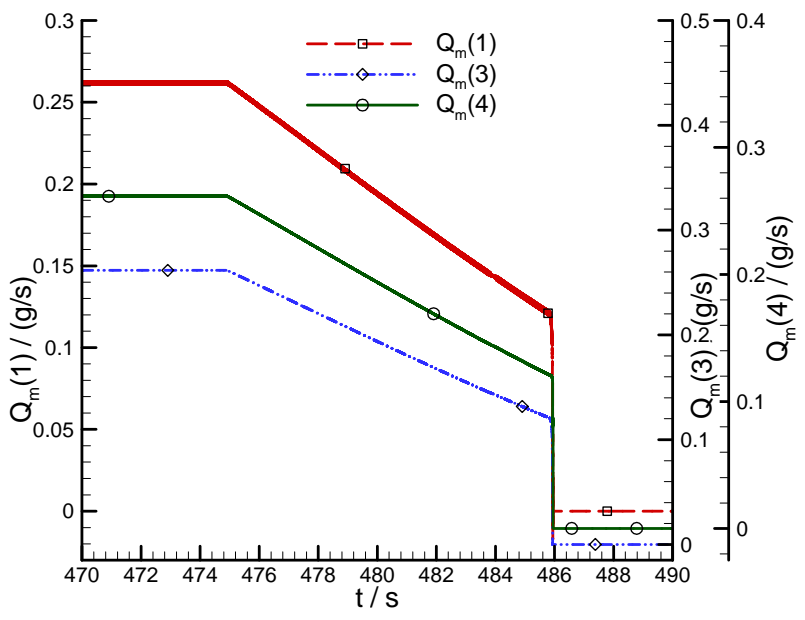

(h) Mass flow rates at three throttling points of CV2 at the shutdown stage of system

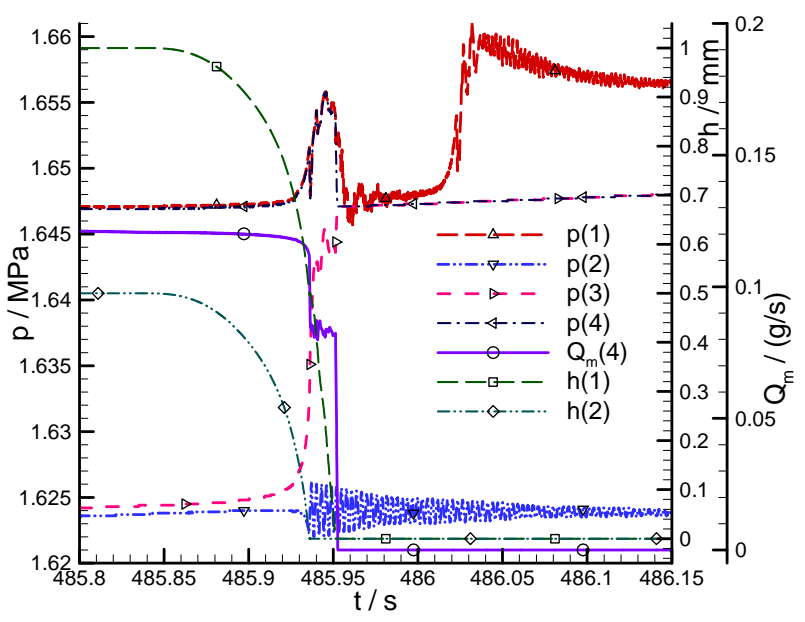

(j) Pressures of four chambers, openings of big and small valve spools, mass flow rate of non-return orifice at the closing stage of $\mathrm{CV} 2$ spools 


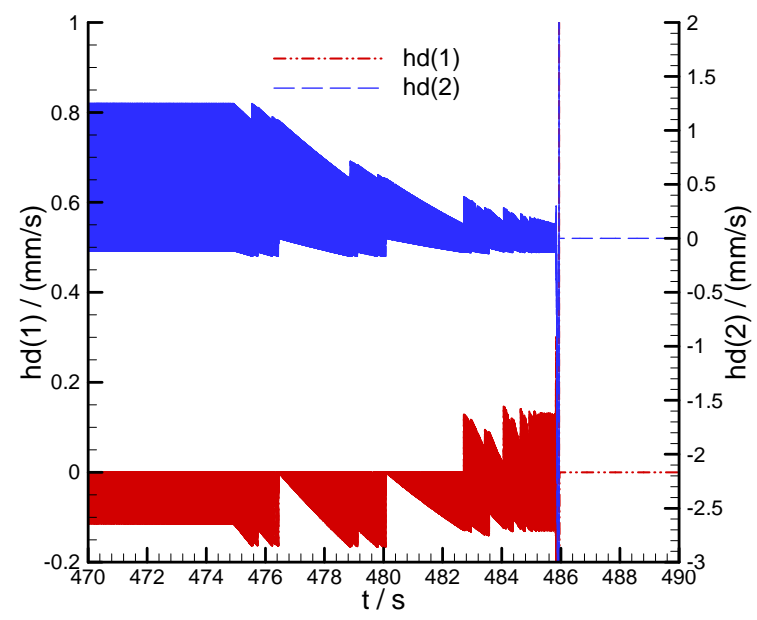

(k) Movement velocities of big and small valve spools at the shutdown stage of system

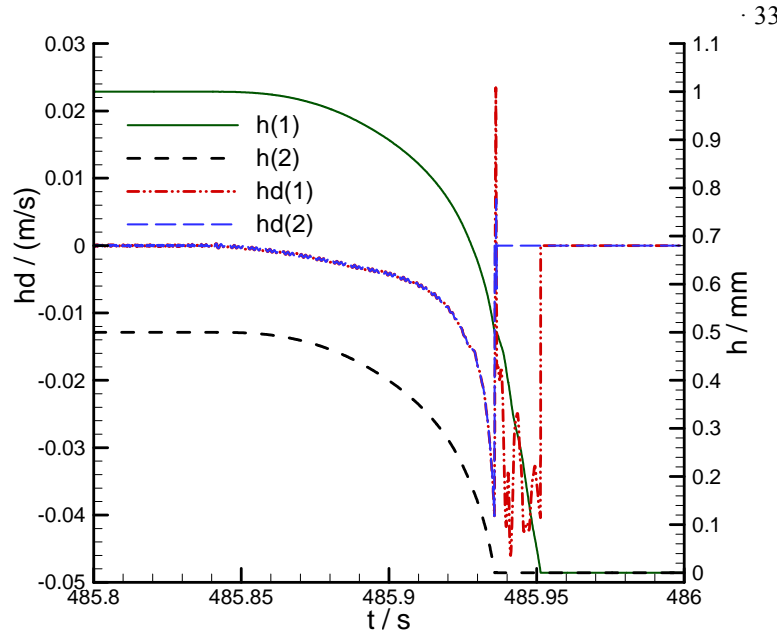

(1) Movement velocities and openings of big and small valve spools at the closing stage of CV2 spools

Fig. 16 Simulation results at four chambers including high-pressure chamber, low-pressure chamber, non-return chamber and opening chamber and three throttling points including big valve spool, small valve spool and non-return orifice of CV2

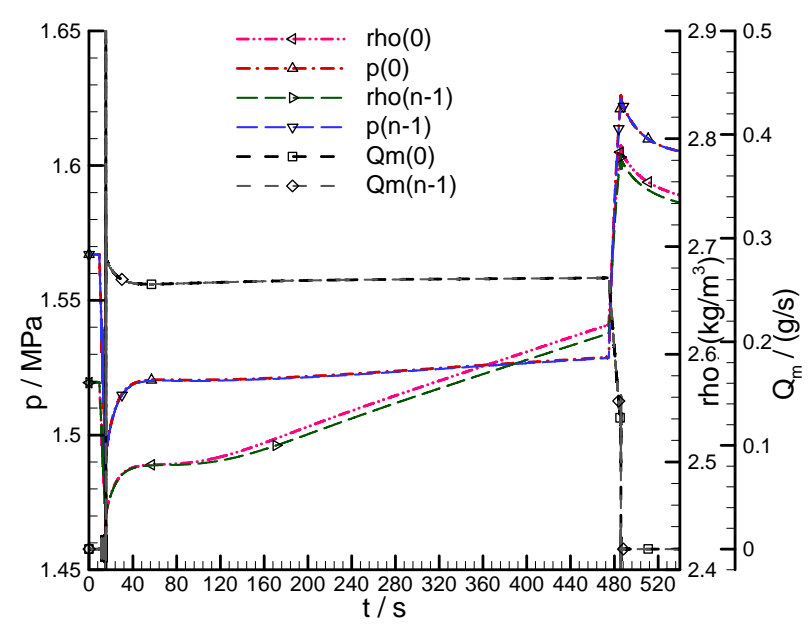

(a) Pressures, densities and mass flow rates

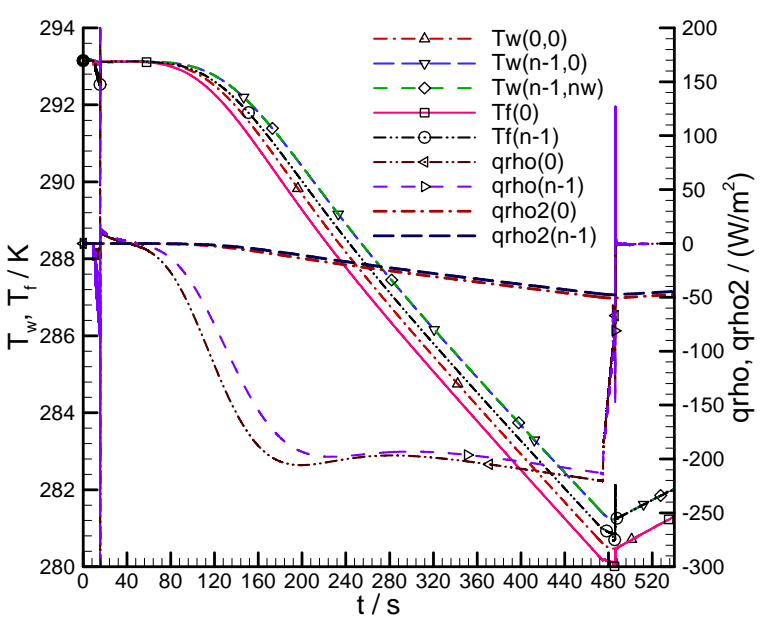

(b) Wall temperatures, fluid temperatures, convective heat flux densities of interior wall and exterior wall

Fig. 17 Simulation results of GP4 entrance and exit grids

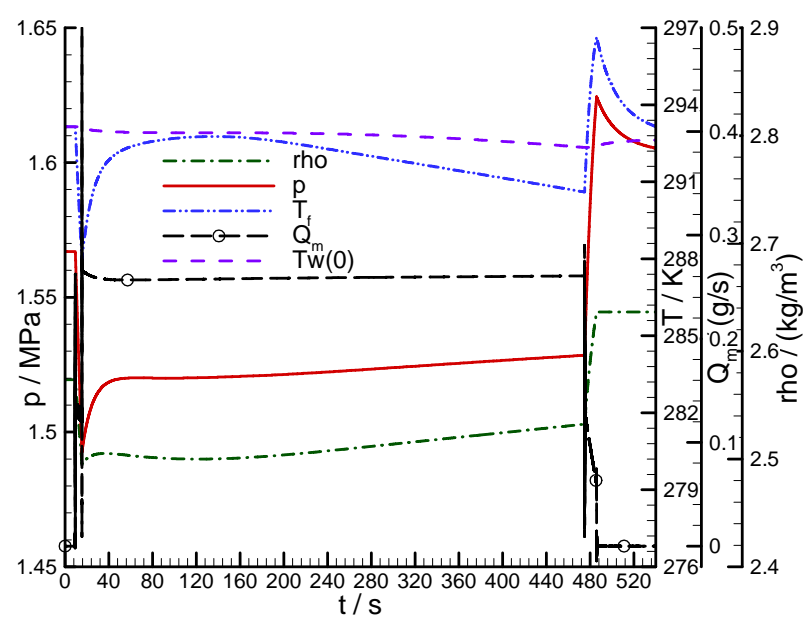

(a) GVol3

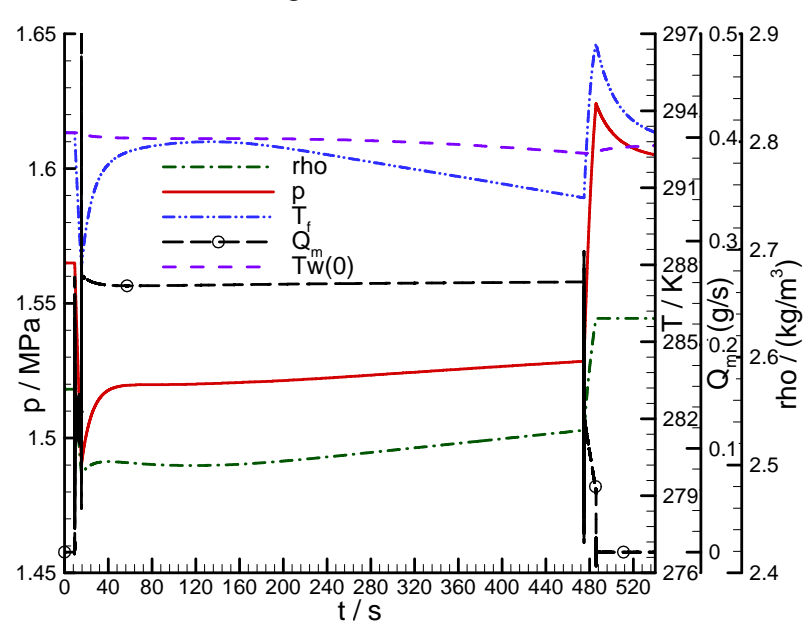

(b) GVol4

Fig. 18 Simulation results of pressures, fluid and wall temperatures, mass flow rates and densities of (a) GVol3 and (b) GVol4 


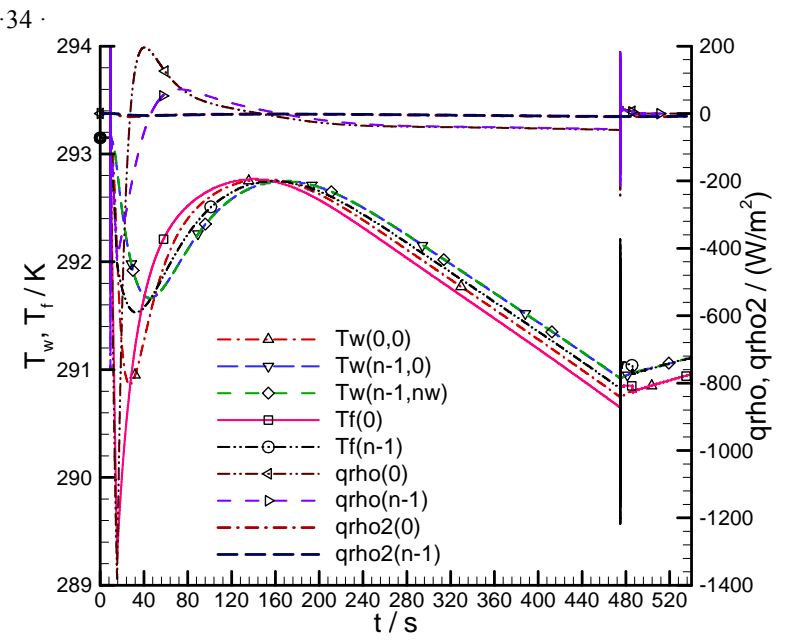

(a) GP5

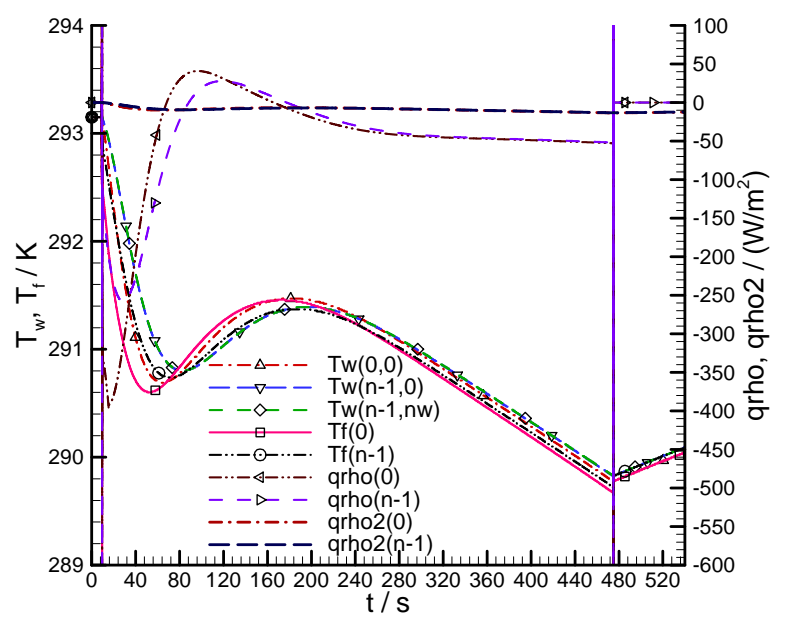

(b) GP6

Fig. 19 Simulation results of wall temperatures, fluid temperatures, convective heat fluxes of interior wall and exterior wall at entrance and exit grids of (a) GP5 and (b) GP6

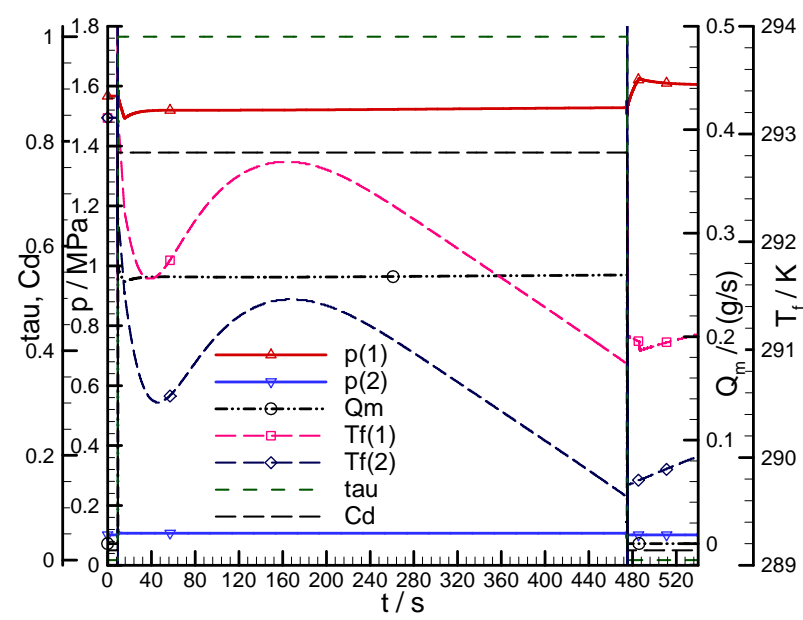

(a) GV1

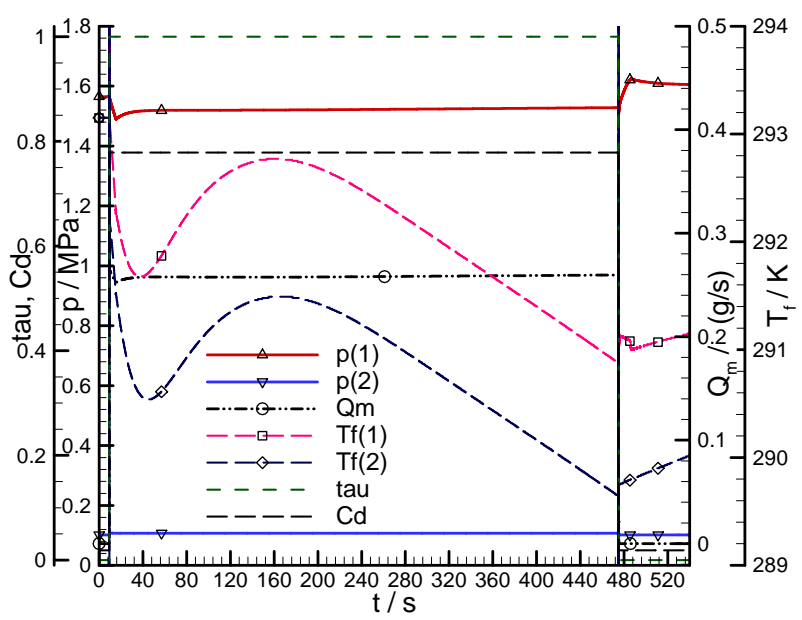

(b) GV2

Fig. 20 Simulation results of valve-spool relative openings and flow coefficients, pressures, mass flow rates, fluid temperatures at entrance and exit grids of (a) GV1 and (b) GV2 


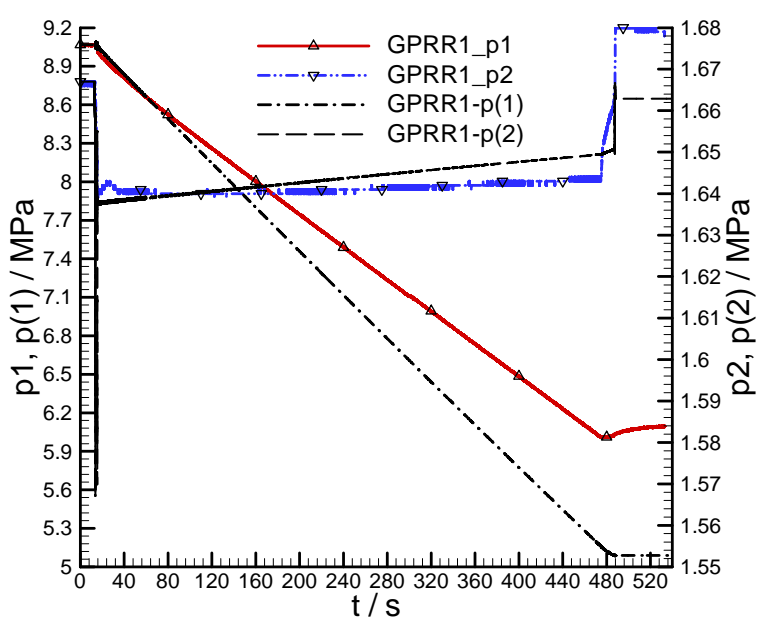

(a) The whole process under the adiabatic condition

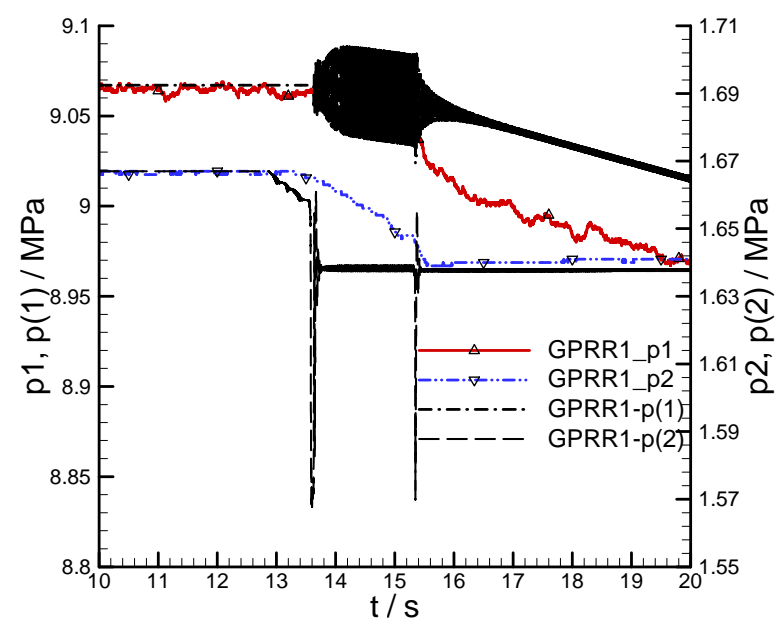

(c) The system startup stage under the adiabatic condition

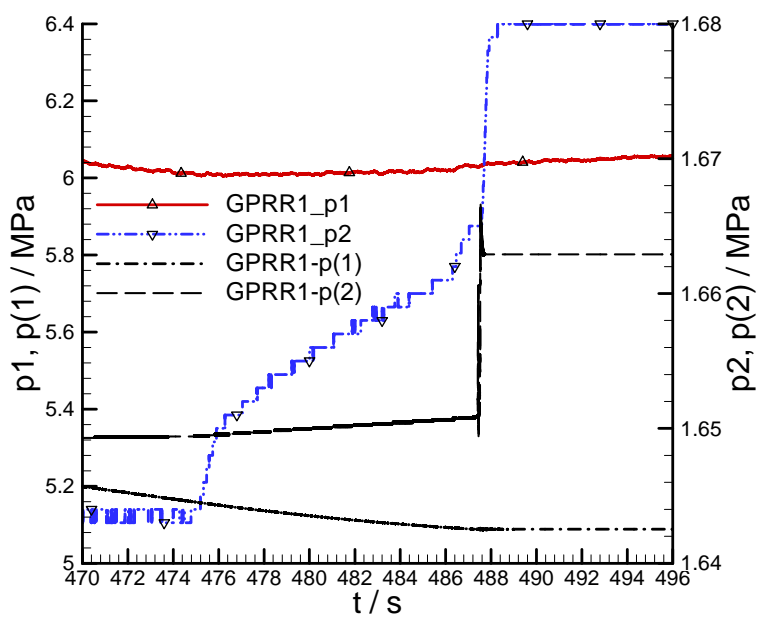

(e) The system shutdown stage under the adiabatic condition

Fig. 21 Comparison of pressure simulation results of GPRR 1 high- and low-pressure chambers with experimental measurements

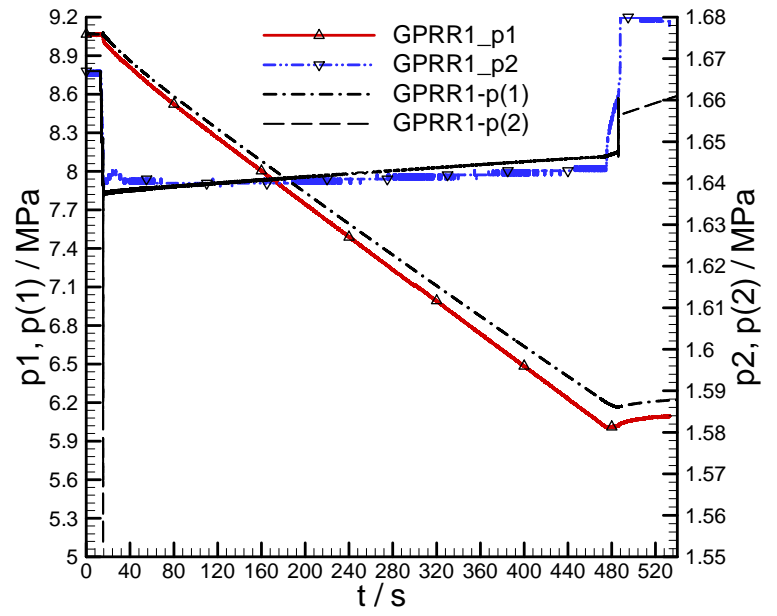

(b) The whole process when considering heat transfer

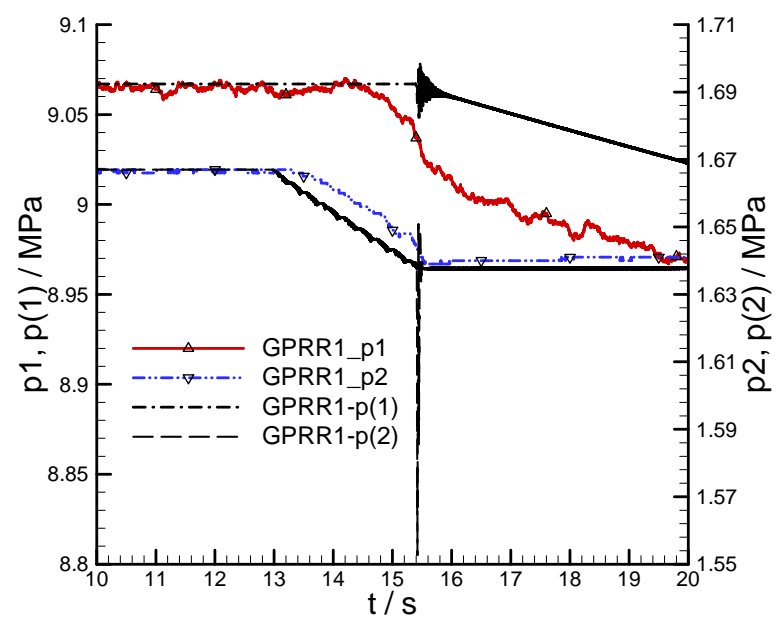

(d) The system startup stage when considering heat transfer

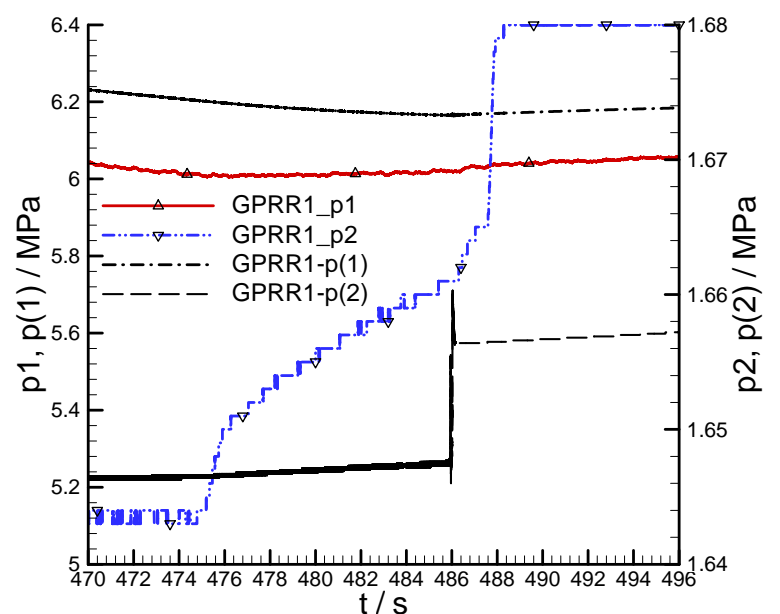

(f) The system shutdown stage when considering heat transfer 


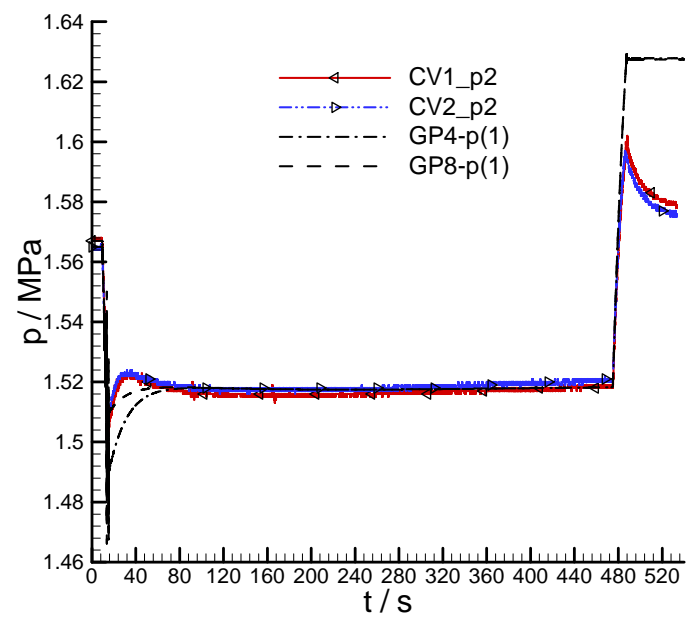

(a) The whole process under the adiabatic condition

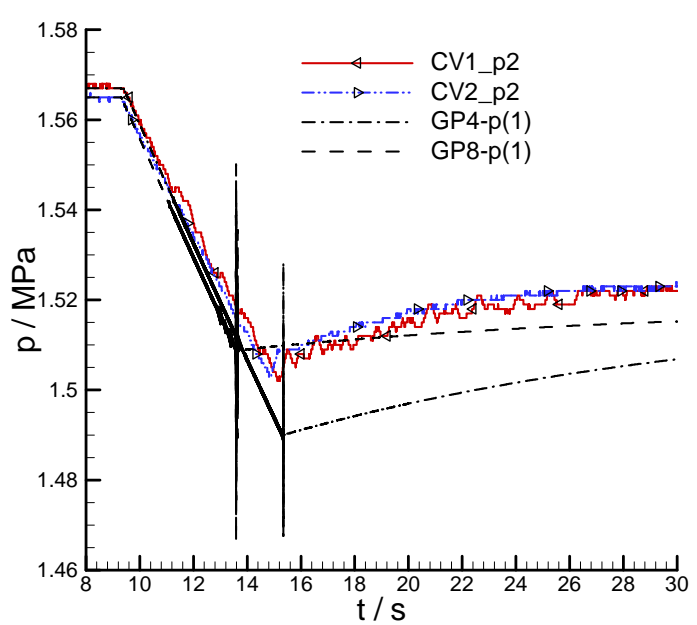

(c) The system startup stage under the adiabatic condition

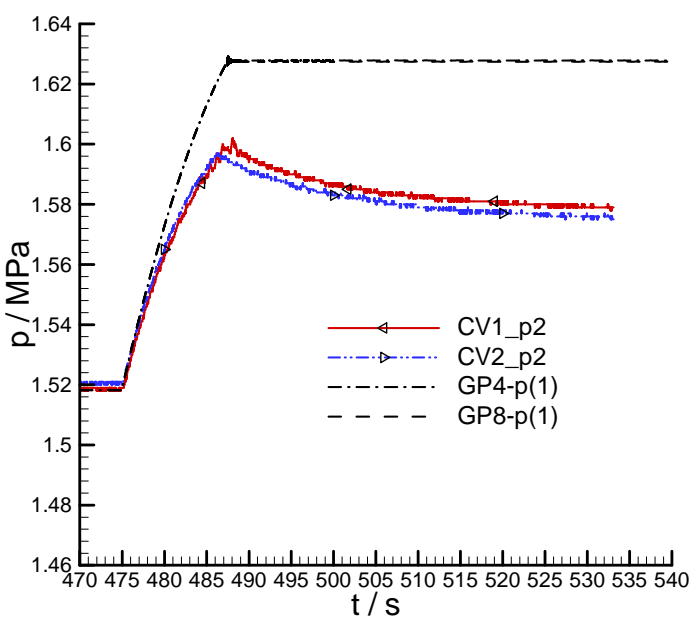

(e) The system shutdown stage under the adiabatic condition

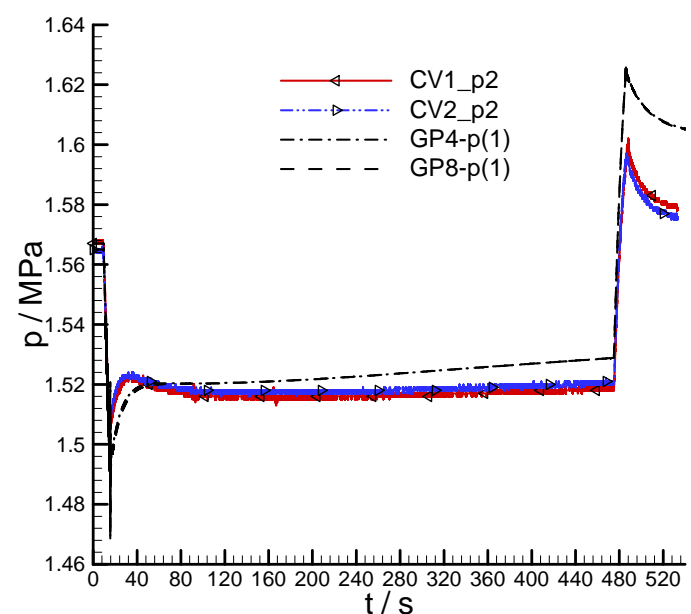

(b) The whole process when considering heat transfer

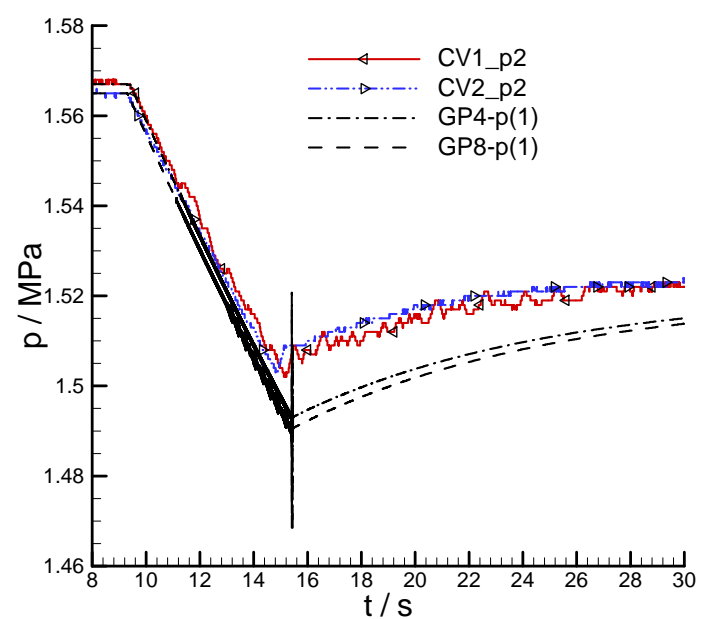

(d) The system startup stage when considering heat transfer

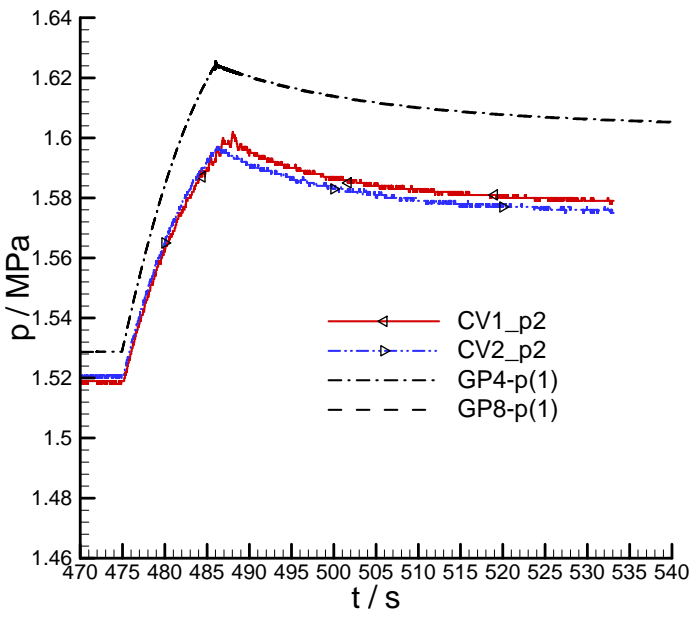

(f) The system shutdown stage when considering heat transfer

Fig. 22 Comparison of pressure simulation results of CV1 and CV2 downstream with experimental measurements 


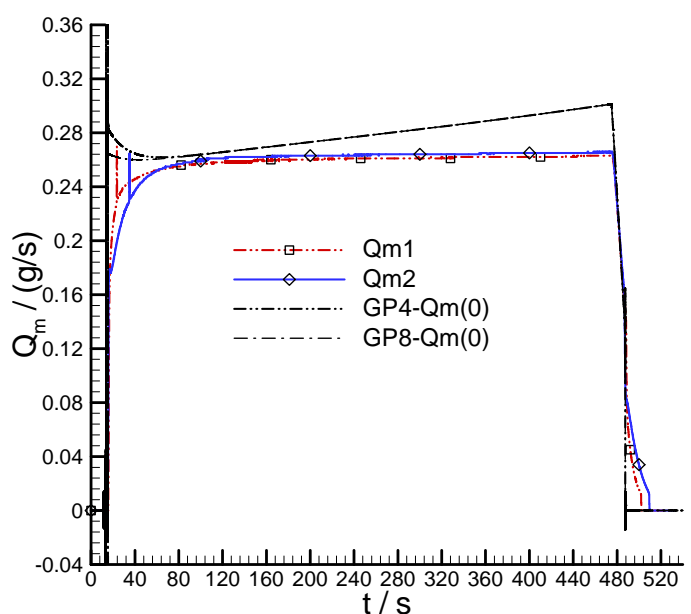

(a) The whole process under the adiabatic condition

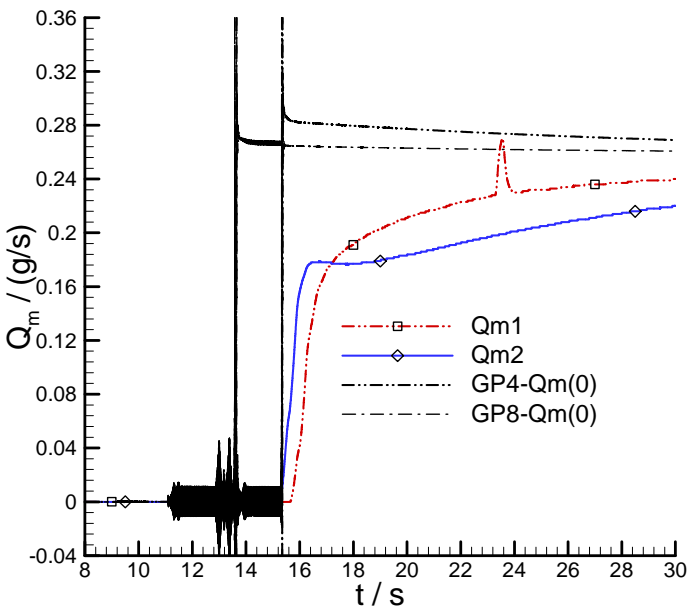

(c) The system startup stage under the adiabatic condition

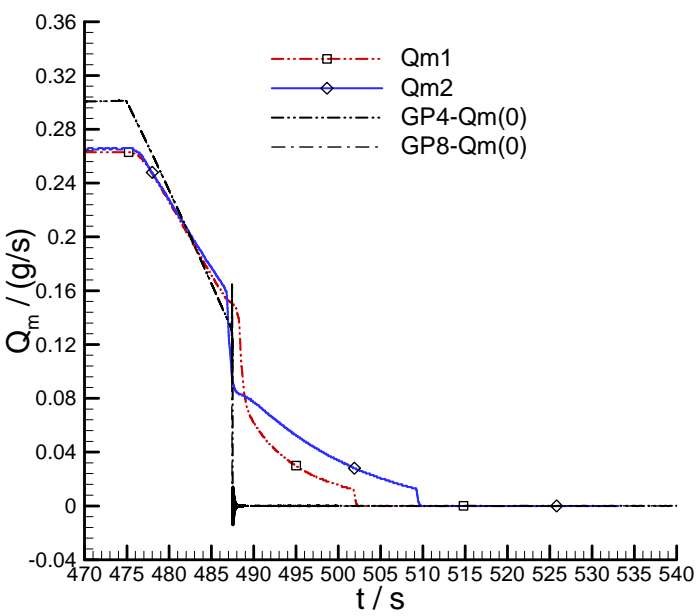

(e) The system shutdown stage under the adiabatic condition

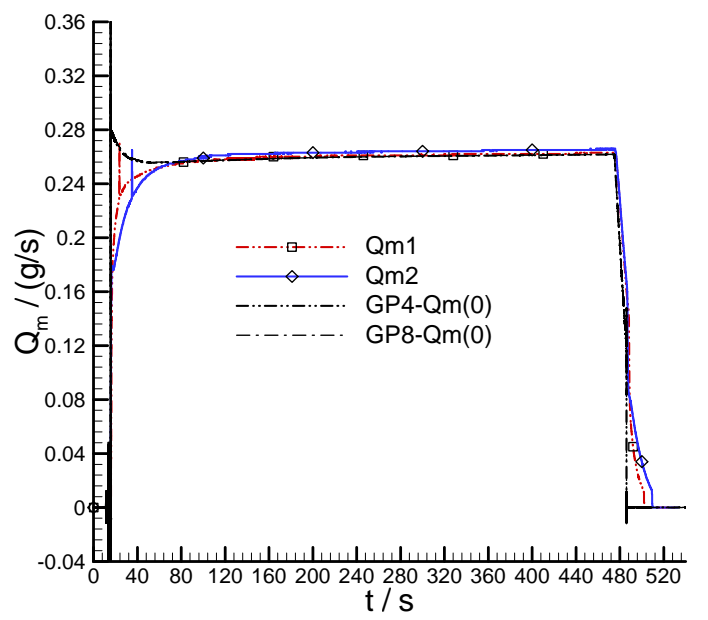

(b) The whole process when considering heat transfer

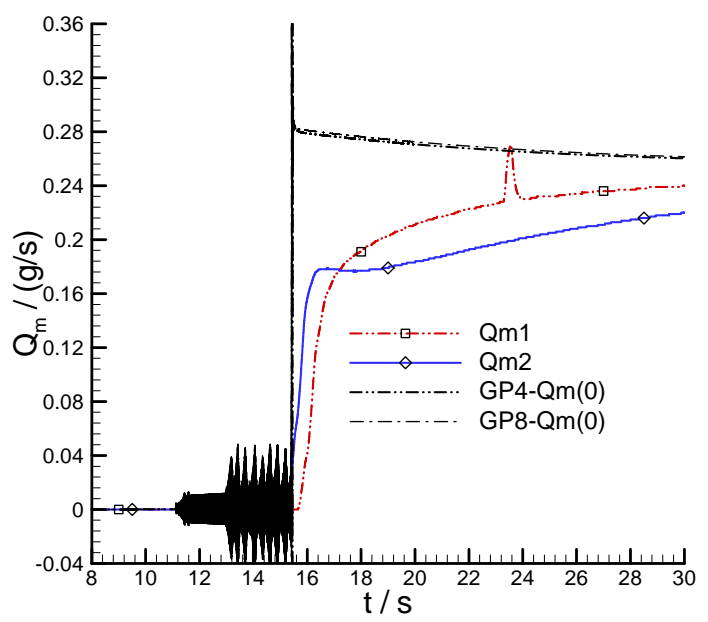

(d) The system startup stage when considering heat transfer

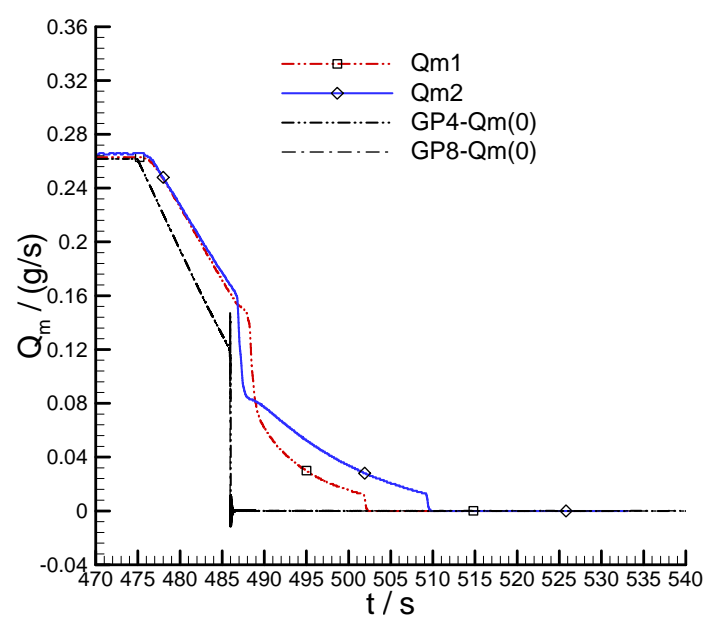

(f) The system shutdown stage when considering heat transfer

Fig. 23 Comparison of mass-flow-rate simulation results of CV1 and CV2 downstream with experimental measurements 


\section{Tables}

Table 1 Modularization disassembly of double-valve combined test system

\begin{tabular}{lcll}
\hline Module type name & Type code & Identifier & Module attribute \\
\hline Fluid Source & 00 & FS & Basis \\
Gas Pipe & 02 & GP & Basis \\
Gas Volume & 12 & GVol & Basis \\
Gas Valve & 22 & GV & Basis \\
Gas Pressure Reducing Regulator & 24 & GPRR & Combination \\
Check Valve & 27 & CV & Combination \\
\hline
\end{tabular}

Table 2 System initial, structure and control parameters settings determined by the test data

\begin{tabular}{|c|c|c|c|c|c|c|c|}
\hline System component & Paramet & s and settin & s of simulati & on case for 9 & IPa working & ondition & Remarks \\
\hline \multirow{2}{*}{ System } & $p_{0} / \mathrm{MPa}$ & $p_{1} / \mathrm{MPa}$ & $p_{2} / \mathrm{MPa}$ & $p_{3} / \mathrm{MPa}$ & $p_{\text {atm }} / \mathrm{MPa}$ & $M_{\Delta t} / \mathrm{s}$ & \multirow{8}{*}{$\begin{array}{l}\text { The adiabatic or the } \\
\text { radial one-dimensional } \\
\text { wall heat transfer model }\end{array}$} \\
\hline & 9.067 & 1.667 & 1.567 & 1.565 & 0.101325 & $10^{-6}$ & \\
\hline \multirow{4}{*}{ GPRR1 } & $d_{\mathrm{v} 3} / \mathrm{mm}$ & $d_{\mathrm{v} 4} / \mathrm{mm}$ & $\lambda_{\mathrm{s}} / \mathrm{s}$ & $C_{\mathrm{d} 1}$ & $C_{\mathrm{d} 3}$ & $C_{\mathrm{d} 4}$ & \\
\hline & 0.3 & 1.0 & $4 \times 10^{-4}$ & 0.9 & 0.05 & 0.05 & \\
\hline & $m_{\mathrm{VC}} / \mathrm{g}$ & $x_{2} / \mathrm{mm}$ & $C_{\mathrm{m}} / \mathrm{N} / \mathrm{mm}$ & $C_{1} / \mathrm{N} / \mathrm{mm}$ & $C_{2} / \mathrm{N} / \mathrm{mm}$ & $C_{3} / \mathrm{N} / \mathrm{mm}$ & \\
\hline & 53.82 & 15.353 & 0 & 7.0 & 137 & 56 & \\
\hline \multirow{2}{*}{$\mathrm{CV} 1$ and $\mathrm{CV} 2$} & $\lambda_{\mathrm{s} 1}, \lambda_{\mathrm{s} 2} / \mathrm{s}$ & $x_{1} / \mathrm{mm}$ & $x_{2} / \mathrm{mm}$ & $C_{\mathrm{d} 1}$ & $C_{\mathrm{d} 3}$ & $C_{\mathrm{d} 4}$ & \\
\hline & $4 \times 10^{-4}$ & 10.532 & 7.6598 & 0.3 & 0.3 & 0.9 & \\
\hline \multirow{7}{*}{ GV1 and GV2 } & \multirow{2}{*}{$d_{\mathrm{vs}} / \mathrm{mm}$} & $C_{\mathrm{d}}$ & \multicolumn{4}{|c|}{ Operating (opening or closing) time sequence } & \\
\hline & & as $\tau_{\mathrm{vs}}=0 \sim 1$ & \multicolumn{2}{|c|}{ Time $t_{\text {test }} / \mathrm{s}$} & Relative o & $\begin{array}{ll}\text { ening } & \tau_{\mathrm{vs}} \\
\end{array}$ & \multirow{6}{*}{$\begin{array}{l}\text { The pressure ratio-based } \\
\text { injector orifice model for } \\
\text { throttling calculation and } \\
\text { the variable-coefficient } \\
\text { default formula scheme } \\
\text { for flow coefficient } \\
\text { calculation }\end{array}$} \\
\hline & GV1, 2 & GV1, 2 & GV1 & GV2 & \multicolumn{2}{|c|}{ GV1, 2} & \\
\hline & \multirow{4}{*}{0.54535} & \multirow{4}{*}{$0 \sim 0.77863$} & 0.0000 & 0.0000 & \multicolumn{2}{|c|}{0.0} & \\
\hline & & & 9.4075 & $\begin{array}{l}9.2077 \\
9.3154\end{array}$ & \multicolumn{2}{|c|}{1.0} & \\
\hline & & & 474.94 & 474.92 & \multicolumn{2}{|c|}{0.6} & \\
\hline & & & 475.20 & 475.13 & \multicolumn{2}{|c|}{0.0} & \\
\hline
\end{tabular}

Table 3 Recovery history of pressures and temperatures of two tanks

\begin{tabular}{c|l|ccccc}
\hline Component & Parameters $\backslash$ Time & $15.41 \mathrm{~s}$ (low point) & $15.44 \mathrm{~s}$ (low point) & $40 \mathrm{~s}$ & $60 \mathrm{~s}$ & $80 \mathrm{~s}$ \\
\hline \multirow{2}{*}{ GVo14 } & Pressure / MPa & 1.4901 & & 1.5177 & 1.5197 & 1.5198 \\
& Temperature / K & 287.91 & & 291.92 & 292.43 & 292.63 \\
\hline \multirow{2}{*}{ GVol3 } & Pressure / MPa & & 1.4925 & 1.5185 & 1.5201 & 1.5200 \\
& Temperature / K & & 287.95 & 291.85 & 292.37 & 292.58 \\
\hline
\end{tabular}

Table 4 Variation history of flow rate of GP4 outlet at the recovery stage of tank pressures

\begin{tabular}{c|ccccc}
\hline Parameters $\backslash$ Time & $15.4444 \mathrm{~s}$ (peak point) & $15.50 \mathrm{~s}$ & $40 \mathrm{~s}$ & $60 \mathrm{~s}$ & $80 \mathrm{~s}$ \\
\hline Mass flow rate $/ \mathrm{g} / \mathrm{s}$ & 1.2563 & 0.2870 & 0.2567 & 0.2556 & 0.2562 \\
\hline
\end{tabular}

Table 5 Pressure distribution of various chamber gases of two check valves before opening and before closing

\begin{tabular}{c|ccccc}
\hline Component & Time & $\begin{array}{c}\text { High- pressure } \\
\text { chamber pressure }\end{array}$ & $\begin{array}{c}\text { Opening chamber } \\
\text { pressure }\end{array}$ & $\begin{array}{c}\text { Non-return chamber } \\
\text { pressure }\end{array}$ & $\begin{array}{c}\text { Low-pressure } \\
\text { chamber pressure }\end{array}$ \\
\hline & $t / \mathrm{s}$ & $p(1) / \mathrm{MPa}$ & $p(4) / \mathrm{MPa}$ & $p(3) / \mathrm{MPa}$ & $p(2) / \mathrm{MPa}$ \\
\hline \multirow{2}{*}{$\mathrm{CV} 2$} & 15.407 & 1.6367 & 1.5648 & 1.5647 & 1.4855 \\
& 485.850 & 1.6471 & 1.6470 & 1.6244 & 1.6238 \\
\hline \multirow{2}{*}{$\mathrm{CV} 1$} & 15.435 & 1.6465 & 1.5668 & 1.5669 & 1.4932 \\
& 485.958 & 1.6472 & 1.6471 & 1.6247 & 1.6241 \\
\hline
\end{tabular}

Table 6 Variation and distribution of system pressures and temperatures at the halt stage

\begin{tabular}{c|l|cccccc}
\hline Time & \multicolumn{1}{|c|}{ Parameters } & GVol1 & GP4 inlet & GVol3 & GP5 outlet & GP6 inlet & GP6 outlet \\
\hline \multirow{3}{*}{$490 \mathrm{~s}$} & He gas temperature / K & 274.11 & 280.52 & 295.93 & 290.97 & 289.84 & 289.89 \\
& Interior-wall temperature / K & 277.95 & 280.53 & 292.45 & 290.98 & 289.84 & 289.89 \\
& Pressure / MPa & 6.1742 & 1.6205 & 1.6205 & 1.6205 & 0.10133 & 0.10133 \\
\hline \multirow{3}{*}{$530 \mathrm{~s}$} & He gas temperature / K & 276.01 & 281.15 & 293.34 & 291.08 & 290.00 & 290.05 \\
& Interior-wall temperature / K & 277.59 & 281.15 & 292.62 & 291.08 & 290.00 & 290.05 \\
& Pressure / MPa & 6.2170 & 1.6063 & 1.6063 & 1.6063 & 0.10133 & 0.10133 \\
\hline
\end{tabular}

